\title{
Complex Dynamics Enabled by Basic Neural Features
}

\section{Dissertation}

\author{
for the award of the degree \\ "Doctor rerum naturalium" \\ of the Georg-August-Universität Göttingen \\ within the doctoral program \\ Physics of Biological and Complex Systems (PBCS) \\ of the Georg-August University School of Science (GAUSS)
}

submitted by

Diemut Regel

from Hildesheim

Göttingen, 2019 
Thesis advisory committee

Prof. Dr. Marc Timme

Network Dynamics, Max Planck Institute for Dynamics and Self-Organization, Göttingen, Germany

Chair for Network Dynamics, Center for Advancing Electronics Dresden (cfaed) and Institute for Theoretical Physics, Technical University of Dresden, Dresden, Germany

Prof. Dr. Reiner Kree

Institute for Theoretical Physics, Georg-August-Universität Göttingen, Germany

Prof. Dr. Florentin Wörgötter

Institute for Theoretical Physics, Georg-August-Universität Göttingen, Germany

Reviewers and members of the examination board

\section{Prof. Dr. Marc Timme}

Network Dynamics, Max Planck Institute for Dynamics and Self-Organization, Göttingen, Germany

Chair for Network Dynamics, Center for Advancing Electronics Dresden (cfaed) and Institute for Theoretical Physics, Technical University of Dresden, Dresden, Germany

Prof. Dr. Reiner Kree

Institute for Theoretical Physics, Georg-August-Universität Göttingen, Germany

Further members of the examination board

Prof. Dr. Florentin Wörgötter

Third Institute of Physics, Georg-August-Universität Göttingen, Germany

Dr. Michael Wilczek

Theory of Turbulent Flows, Max Planck Institute for Dynamics and Self-Organization, Göttingen, Germany

Prof. Dr. Uwe Mattler

Department of Experimental Psychology, Georg-August-Universität Göttingen, Germany

Prof. Dr. Peter Sollich

Institute for Theoretical Physics, Georg-August-Universität Göttingen, Germany

Department of Mathematics, King's College London, UK 


\section{Contents}

1 Introduction 1

2 Theoretical Background 7

2.1 Rules of Change - Dynamics . . . . . . . . . . . . . . . 7

2.2 Topological Structures in Phase Space . . . . . . . . . . . . 8 8

2.3 Bifurcations . . . . . . . . . . . . . . . . . 10

2.4 Dynamics on Networks as a Special Case of Dynamical Systems . . . . 10

2.5 Important Theorems . . . . . . . . . . . . . . . . . . . . 111

3 Nested Limit Cycles in a Minimal Adaptive System 13

3.1 Introduction . . . . . . . . . . . . . . . . . . 13

3.2 The Original System . . . . . . . . . . . . . . . . . . . 15

3.2 .1 System Definition . . . . . . . . . . . . . . 15

3.2.2 A Numerical Study of the System's Behavior . . . . . . . . . 16

3.3 The Zigzag-Potential - a Simplified System . . . . . . . . . . . . . 16

3.3 .1 System Definition . . . . . . . . . . . . . . 16

3.3.2 Analytic Segment-Wise Solutions . . . . . . . . . . . . . 16

3.3.3 Proof for Finite Time Convergence to Limit Cycles - Core Ideas 21

3.3.4 Proof for Finite Time Convergence to Limit Cycles - Detailed

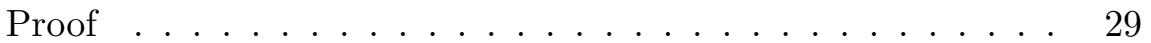

3.4 Discussion . . . . . . . . . . . . . . . . . $\ldots \ldots$

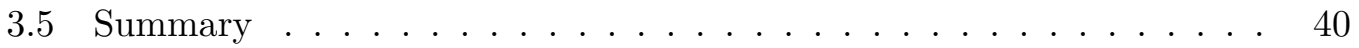

4 Reordering and Order Conservation in Symmetric All-to-All Pulse-Coupled Phase Oscillator Networks Depending on Self-Loop Strength 43

4.1 Introduction . . . . . . . . . . . . . . . . . . . 43

4.2 Breaking of Order Conservation by Removing Self-Loops in a Smooth

Pulse-Coupled System . . . . . . . . . . . . . . . . 46

4.2 .1 System Definition . . . . . . . . . . . . . 47

4.2.2 Transition to Order Conservation with Self-Loops . . . . . . . . 47

4.3 Analysis of Reordering Processes in a Spiking System with Self-Loops 49

4.3 .1 System Definition . . . . . . . . . . . . . . . 49

4.3.2 Return Map for Phase Differences . . . . . . . . . . . . 51

4.3 .3 A Folding Mechanism . . . . . . . . . . . . . . 56

4.3.4 The Transition to Order Conservation with Self-Loops . . . . . 58

4.3.5 Reordering Patterns within One Cycle . . . . . . . . . . . . 60 60

4.3.6 Reordering Patterns Across Cycles . . . . . . . . . . . . . 62 62

4.4 Summary and Discussion . . . . . . . . . . . . . . 65 65

5 Chaos in Two Pulse-Coupled Phase Oscillators 71

5.1 Introduction . . . . . . . . . . . . . . . . 71 
5.2 System Definition . . . . . . . . . . . . . . . . 73

5.3 Signature of Chaos . . . . . . . . . . . . . . . . . 774

5.4 Source of Chaotic Behavior . . . . . . . . . . . . . 779

5.5 Discussion . . . . . . . . . . . . . . . . . 82

5.6 Summary . . . . . . . . . . . . . . . . 85

6 Discussion 8

6.1 Theoretical Considerations on Restrictions and Freedom in Phase Space 88

6.2 Dynamical Neural Features and Phase Space Constraints . . . . . . . 91

6.3 The Approach of Studying Example Systems . . . . . . . . . . . . . 92

6.4 Introducing Neural Features Does Not Always Change Everything . . 94

7 Summary 97

$\begin{array}{ll}\text { Appendix } & 101\end{array}$

$\begin{array}{lr}\text { Bibliography } & 107\end{array}$

Acknowledgements 


\section{Chapter 1}

\section{Introduction}

The human brain is considered by many to be the most complex object we know. It enables us to perceive, to think, to feel and to interact with the world. Understanding how the brain works broadens our perspective on who we are, may provide us with better treatment options for mental and neurological disorders and may enable new technologies in the field of artificial intelligence. At the same time understanding the brain is a daunting task. The brain encompasses a massive amount of interacting entities operating on several spatial and temporal scales. While there is relative good experimental access to small spatial scales on the level of cells and cell compartments as well as on the scale of the whole brain, e.g. through neuro-imagine techniques such as fMRI or EEG, the mesoscopic scale concerning the neural network level is still relatively difficult to access [69, 78. In theoretical neuroscience researchers often create complex models consisting of larger networks which produce a broad range of complex dynamical behavior [12, 36, 151, 59, 76, 88, 89]. The neural units of these networks typically involve features that are specific to biological and especially neuronal systems and not common to systems of classical physics. As a result their dynamical implications are less studied and less understood.

The goal of this thesis is to further our understanding of the fundamental dynamical properties of neural features such as adaptation and pulse-coupling with and without delay. We ask, which qualitative new dynamics can arise, if these features are included into minimal dynamical systems. We approach this question by studying three example systems and find remarkably rich dynamics.

\section{What is a Good Model?}

A good model is simple. A good model, just as in every science, is a model that is complex enough to produce the targeted empirical observations while being as simple as possible. Already Occam in the fourteenth century [9] proposed the idea of choosing the simplest of all models which can explain the behavior. His idea was that the simplest model with the least amount of assumptions necessary to explain something is more likely to be true. The more assumptions and complexity is added, the more likely it is that one adds a wrong assumption, which renders the model wrong.

There is another reason for choosing the simplest model: In a trivial sense the best model to explain something is the object itself - the brain is the best model for the brain. However, even if somebody manages to read out every detail of a brain, already a daunting task, and produces a gigantic computer simulation which produces 
output just as a real brain would, the question arises what has been learned (see also 'human brain project' [53]). It seems the person has succeeded in including everything necessary to emulate a brain, so no essential ingredient was forgotten. At the same time, this model would not provide us with the satisfying feeling of understanding how the brain works. We are not provided with a mechanism or principle that produces the observed complexity but left with the complexity itself. Given such complexity of brain structure and dynamics one might have the intuition that the building principle of the brain must be complex in itself. We would like to challenge this intuition: First, there are many example systems which have demonstrated that a set of extremely simple rules is sufficient to produce remarkably complex behavior. An early example of this kind is Langton's Ant [92] (and turmites as systems following the same spirit) and chaotic systems such as the Lorenz or the Roessler attractor [49, 72]. Langton's ant describes an agent, the ant, living on a square lattice. Each time the agent reaches a white cell, it turns 90 degrees, flips the color of the cell to black and moves one step forward. If it reaches a black cell, it moves 90 degrees to the left, flips the color of the cell to white and moves one step forward. Despite these extremely simple rules the emerging movement does not seem to settle into any graspable pattern for a long time. The Lorenz and the Roessler attractor are both systems of only three simple ordinary differential equations. The resulting behavior of each system, even though fully deterministic, appears to be random. Second, the brain has a remarkable ability to self-organize. Examples that show that the brain's organization is not hard-wired include the visual system taking over other functions not related to vision in congenital blind humans (see reference [40] for a review). We have shown that self-organizing systems can show very complex dynamics arising from extremely simple rules and that the brain has large self-organizing capabilities. Given these two arguments it is reasonable to assume that the complex behavior of the brain may originate from much simpler principles. While not a given, there is hope that these principles are simple enough that they can be grasped by our human intellect.

The goodness of a model depends on the research question. Theoretical neuroscience aims at developing models to understand behavior observed in the brain. This behavior can range from specific measurements of single cell spike trains or membrane voltage fluctuations on the small scale to large scale brain measurements like fMRI or EEG data or human behavior. The empirical phenomenon to be explained may also vary on the time scale from a few milliseconds (e.g. the generation of spike, large voltage fluctuations in membrane potential) to seconds and beyond (e.g. fMRI data or human behavior).

There is not a single good model for the brain, because the goodness of the model does not only depend on its simplicity but at the same time on the observable to be explained. A detailed model of a single neuron may be a good model for explaining membrane potential fluctuations across the membrane, whereas at the same time it may be a bad model for large scale observables, such as EEG data. To understand the principles behind EEG data a detailed neuronal model may need large resources for simulation. And more importantly the basic principles responsible for the targeted observable cannot be dissected from additional ingredients included in the model but not key to the observed behavior. While the model can be useful to make predictions 
it lacks the beauty of providing a moment of insight, the feeling of reducing something complex to something much simpler to grasp.

The mesoscopic scale of the brain is difficult to access empirically. Often models use entities from a more fine grained level of resolution and combine them in a simple way to produce the observed behavior on a more coarse grained level of resolution. This works well if the gaps between the resolutions that can be experimentally accessed are not too large.

Neuroscience is a vast field ranging across several orders of temporal and spatial scales. Whereas some of them can be accessed well experimentally, others still lack good measurement devices.

There is relatively good experimental access to smaller scales such as single neurons and subneuronal parts [78] and scales of the order of the brain as a whole, good methods to provide a direct window into the mesoscopic scale of larger neural networks are rare [69. Although, some methods have been developed (e.g. calcium imaging, multielectrode arrays). However these methods capture only a small subset of the network components, they only sample a fraction of neurons or do not have any or very limited knowledge of the connectivity between neurons or other parameters are missing [43, 75, 178. This leaves the theoretical community with many degrees of freedom as to how to design a neural network model producing the behavior observed at the larger scale. Accordingly, many very different neural network models have been proposed [13, 29, 88] and the field is far from converging onto a commonly agreed upon model class. For example if one is interested in understanding the computational aspects of the brain, it is not even clear whether the computation is done based on the level of firing rates (e.g. [48]) or single spikes (e.g. [29]) or some combination. While the task at hand seems daunting some proposed models provide hope, e.g. the balanced state model [88, 89] explains essential behavior observed in neural networks, including self-sustained irregular firing, while being relatively independent of the actual neural model used, effectively reducing the degrees of freedom because many different neural models converge to the same qualitative behavior.

The emergent behavior of neuronal networks in the brain is complex, eventually it seems to be the basis for everything we perceive, think, feel or do. This has lead to many neural network models, consisting of several neurons producing complex behavior. However the neurons in these networks often are already complex themselves including dynamical features such as adaptation, delay and pulse-coupling [56]. This is justified experimentally in the sense that real neurons themselves show dynamically very complex behavior [5, 20, 22, 33]. As demonstrated above, the best model is not necessarily the one including all the complexity of the units used as building blocks for the model. Instead, if one aims at understanding the fundamental principles behind observed behavior reducing the model as far as possible to get to the core ingredients necessary for the to be explained behavior may proof beneficial. When considering larger networks showing complex behavior, it is not clear whether to attribute the complexity to network effects or to the complexity of the neural model used as building blocks of the network. Here we contribute to the dissection process by analyzing the effects of dynamical features often included into neural models used in larger scale 
neural networks.

While these features are very common to models in neuroscience or other biological systems, they only rarely occur in systems of classical physics. Dynamical system theory is concerned with the relation between equations providing a rule of how variables change in time and the topological structure of the resulting trajectories in phase space (the space spanned by these variables) [82]. It was developed while studying physical systems, such as planetary movements, and has become very successful in describing such systems. However, dynamical features common only to biological, in particular neuronal, systems, are far less studied and far less understood from a dynamical systems' perspective.

In this thesis, we explore the dynamical properties of adaptation and pulse-coupling with and without delay by considering three extremely simple systems including these dynamical features and observe the complexity that these systems can produce from a dynamical systems perspective.

\section{Adaptation as a Neural Feature}

Many models of neurons include adaptation [20, 23, 56]. The first system we consider provides an example of a very simple adaptive system. Adaptation is not a formally well defined concept, but there are systems that are generally considered to be adaptive, e.g. species changing their features as a result of environmental changes (famous examples include Darwin's finches, the peppered moth and bacteria becoming resistant to antibiotics [91, 93]) or neuronal firing rates eventually reducing in response to the extended presentation of a stimulus [23. In both cases one entity optimizes itself to better suit changes in the surroundings. In the first case the phenotype of a species changes due to changes in the living environment optimizing survival. In the second case neurons adjust their firing rate to changes in the baseline input, thereby reducing energetically expensive spike generation.

In chapter 3 we study adaptation. We understand adaptation as the process of an entity following the gradient of a potential to minimize energy expenditure within its environment. At the same time the environment represented by the potential changes on a slower time scale. We propose a minimal such system, that is fully described by only two ordinary differential equations. The phase space is characterized by limit cycles nested within each other. A proof for the limit cycle behavior is provided for a simplified system showing the same qualitative behavior.

Interestingly, due to the system's simple set-up it relates to other research fields that are not concerned with adaptation but also converge onto similar systems or

equations. A discussion of the system in different contexts is provided at the end of chapter 4 .

\section{Pulse-Coupling with and without Delay as a Neural Feature}

The other two systems studied in chapter 4 and 5 focus on the effects of pulsecoupling between network cells as opposed to interactions fully described by ordinary differential equations. 
Chemical synapses are the most common form of connection between neurons [94]. Typically, an action potential, a large fluctuation in the neuron's membrane potential, is generated at the soma, the cell body of a neuron. It then travels along an axon, a cabel-like structure, to the axon terminals, the neuron's outward connections to other neurons. Here, at the presynaptic side of the synapse, the action potential leads to a release of neurotransmitters, chemical messengers, into the synaptic cleft, a small gap between the two connected neurons. These neurotransmitters reach the other side of the synaptic cleft, cause ion channels to open, leading to a change in membrane potential of the postsynaptic neuron.

In many neural network models each neuron is modeled as a single dynamical variable describing the membrane potential. When the membrane potential reaches a threshold a pulse is sent to all postsynaptically connected neurons. Each incoming pulse to a neuron adds a predefined kernel to the total input. These kernels can vary in shape, e.g. starting at zero and be fully differentiable (e.g. [38]) or include discontinuities, delays or having the shape of a $\delta$-function (e.g. [5, 36, [51]). Even though pulse-coupling is very commonly used in network models in theoretical neuroscience a systematic understanding from a dynamical systems perspective is missing [5, 38]. We argue in line with Kielblock et al. 38, that pulse-coupling removes restrictions posed on possible trajectories in phase space allowing for richer dynamics as can be obtained by similar systems described solely by ordinary differential equations.

In chapter 4 we consider an all-to-all symmetrically coupled phase-oscillator network. Symmetries often produce rich dynamics [8, 11, 24, 30, 62, 81, 85. While Golubitsky et al. 25. established that such a system described by ordinary differential equations necessarily shows order conservation, Kielblock et al. [38] showed that this is not true for pulse-coupled system. Building on their work we study the transition to regaining order conservation by gradually introducing self-loops into the pulse-coupled system and provide an analytic understanding for the reordering process. The implications for phase space topology by introducing pulse-coupling and self-loops into a symmetrical system are discussed.

In chapter 5 we demonstrate that a network of only two $\delta$-pulse-coupled phase oscillators shows chaotic behavior while the Poincaré-Bedixson-Theorem only allows for chaos with at least three oscillators in a system of ordinary differential equations. Again this is an example that introducing a neural feature into a simple dynamical system gives rise to complex behavior, already very small neural networks can be chaotic.

\section{Thesis Structure}

In Chapter 2 the theoretical background is introduced and important concepts are defined. In Chapter 3, 4 and 5 we present three systems that show complex behavior arising from basic neural features. Chapter 3 concerns a two dimensional adaptive system captured by two ordinary differential equations. A proof for the numerically observed phase space structure of nested limit cycles is presented for a simplified version of the system. The system in chapter 4 consists of symmetrically all-to-all pulse-coupled phase oscillators. The transition to order conservation by introducing self-loops and the reordering process of the oscillators is studied analytically. Chapter 
5 presents a network of only two pulse-coupled neural phase oscillators showing chaotic behavior. In chapter 6 and 7 the results are discussed and summarized. 


\section{Chapter 2}

\section{Theoretical Background}

Dynamical system theory is concerned with the relation between equations providing a rule of how variables change in time, the dynamics, and the topological structure of the resulting trajectories in phase space, the space spanned by these variables.

In this chapter we will provide the theoretical background, which the rest of the thesis is build upon. We will introduce many important concepts of dynamical system theory, however, this chapter cannot and does not aim at providing an exhaustive introduction. We will only touch upon many concepts, focusing on dynamical features, that are most relevant for the following work.

\subsection{Rules of Change - Dynamics}

The rules describing the temporal evolution of a system are either continuous in time, described by differential equations, or discrete in time, described by difference equations, also known as iterated maps.

Time-continuous systems and ordinary differential equations (ODEs). Dynamical systems only have a single independent variable, time, therefore all equations describing a dynamical system are ordinary and not partial differential equations. An ordinary differential equation, we will use $O D E$ as an abbreviation, has the form

$$
\dot{x}=f(x, t) .
$$

The one dimensional system corresponding to this equations is non-autonomous. If the explicit time dependence is dropped, it is called autonomous. A dynamical system consists of a set of equations, each describing the evolution of one dynamical variable in the system. For an n-dimensional autonomous dynamical system with time-continuous dynamics the equations are the following:

$$
\dot{\mathbf{x}}=\mathbf{f}(\mathbf{x}) .
$$

Time-discrete systems. An iterated map has the form

$$
x(a+1)=g(x(a)) .
$$


An n-dimensional time-discrete dynamical system has the following form:

$$
\mathbf{x}(a+1)=\mathbf{g}(\mathbf{x}(a)) .
$$

A system, that comprises of continuous as well as discrete dynamics is called hybrid system. An example is a neural model, which has continuous membrane potential dynamics until it reaches a threshold, spikes and resets, which are discrete events.

Phase space. The phase space encompasses all possible states of a dynamical system. Usually it is the space spanned by all dynamical variables $\left.\left\{x_{1}, x_{2}, \ldots, x_{n}\right\}\right)$. As we will discuss in chapter 4,5 and 6 , the dynamical variables are not always sufficient to fully describe the system state at a given moment in time. Additional dimensions may become necessary, e.g. as we will see pulse-coupling induces additional dimensions, since a pulse sent in the system's past may still have an effect on the system's future. Here, we will distinguish between the space that is spanned by all dimensions necessary to describe the system's state, the phase space, and the space spanned by the dynamical variables alone. In an autonomous system of ODEs the two spaces coincide.

Initial Conditions. The initial conditions encompass all the information necessary to fully determine the system's future from a given starting point $t_{0}$ in time. They include the values of all dynamical variables at time $t_{0}\left(\left\{x_{1}\left(t_{0}\right), x_{2}\left(t_{0}\right), \ldots, x_{n}\left(t_{0}\right)\right\}\right)$. Additional information may be required, e.g. pulse-coupled systems may require information on pulse-sending times with $t<t_{0}$, since the corresponding pulses may still influence the system's future (see chapter 4,5 and 5 ).

Trajectories and flow. A trajectory $x(t)$ is a set of system states, that is ordered in time. The flow $\Phi(x)$ of a time-continuous dynamical system is a vector field indicating the change anywhere in phase space. The dynamical equations directly provide the flow for each system state: $\dot{\mathbf{x}}=\mathbf{f}(\mathbf{x})=\Phi((x))$.

\subsection{Topological Structures in Phase Space}

Dynamical system theory is particularly interested in phase space topology. Two objects are topologically equivalent, if a continuous transformation exists, that can change one into the other. Continuous transformations include stretching, bending and twisting, but not cutting or glueing. What kind of topological structures can be produced by different dynamical systems? In the following we will introduce some key structures.

Fixed points. A trajectory passing through a fixed point will remain at this point forever. At a fixed point $\mathbf{x}^{*}$ of a time continuous system the dynamical flow is zero $\left(\mathbf{f}\left(\mathbf{x}^{*}\right)=0\right)$. In a time discrete system the map returns the same point in the next 
iteration step: $\mathrm{x}^{*}=\mathbf{g}\left(\mathrm{x}^{*}\right)$.

A fixed point is stable, if all trajectories starting within a small vicinity around this point will approach it as time goes to infinity. If all trajectories in a small vicinity around the fixed point leave the vicinity the fixed point is unstable.

For time-continuous systems stability can be determined in the following way: First, the system can be rewritten as a taylor expansion around the point $\mathbf{x}^{*}$ :

$$
\dot{\mathbf{x}}=\mathbf{f}\left(\mathbf{x}^{*}\right)+J \delta \mathbf{x}+\mathcal{O}\left(\delta \mathbf{x}^{2}\right) .
$$

If all eigenvalues of the Jacobian matrix $J$ at point $x^{*}$ are negative, the fixed point is stable. If all eigenvalues of $J$ are positive, the fixed point is unstable. If all eigenvalues of $J$ are equal to zero, the fixed point is marginally stable.

Now let us consider time-discrete systems: Consider a small perturbation $\delta \mathbf{x}$ to the fixed point $\mathbf{x}^{*}$. Does this perturbation grow or shrink over iterations of the map? The perturbation after one iteration becomes

$$
g\left(\mathbf{x}^{*}+\delta \mathbf{x}\right)-g\left(\mathbf{x}^{*}\right)=g\left(\mathbf{x}^{*}\right)+J \delta \mathbf{x}+\mathcal{O}\left(\delta \mathbf{x}^{2}\right)-g\left(\mathbf{x}^{*}\right) \approx J \delta \mathbf{x} .
$$

If all eigenvalues $|\lambda|<1$ of the jacobian matrix $J$ the fixed point is stable, if all eigenvalues $|\lambda|>1$, the fixed point is unstable. If all eigenvalues $|\lambda|=1$ the fixed point is marginally stable.

Periodic Orbits. If a trajectory always passes through the same points repeatedly, then it is called a periodic orbit. More formally, if there exists a time interval $T$ such that $\tilde{\mathbf{x}}(t+T)=\tilde{\mathbf{x}}(t)$ for all $t$ then the set $\tilde{\mathbf{x}}$ is a periodic orbit or periodic cycle. If all neighboring trajectories approach the periodic orbit it is called a stable limit cycle. If all neighboring trajectories move away, it is called unstable limit cycle. If the neighboring trajectories are periodic orbits as well, the periodic orbit is not a limit cycle and is marginally stable.

Chaos. While there is no overall accepted definition of chaos, there are three characteristics that every chaotic system shares (according to reference [82], p. 331):

1) trajectories do not settle into fixed points, periodic orbits or quasi-periodic orbits. Instead the long term behavior is irregular and does not follow a fixed pattern. Note that irregular long term behavior excludes the possibility of trajectories approaching infinity as $t \rightarrow \pm \infty$. The state space of a chaotic system is bounded.

2 ) the system is deterministic, there is no noisy input or randomness involved.

3 ) the system depends sensitively on initial conditions. Trajectories starting arbitrarily closed to each other will exponentially diverge, this translates into the system having at least one positive lyapunov exponent. 


\subsection{Bifurcations}

If the topological structure of the phase space changes qualitatively due to the variation of a system's parameter, this is called bifurcation. For example fixed points may appear or a fixed point may become a limit cycle at a specific parameter value, the bifurcation point.

\subsection{Dynamics on Networks as a Special Case of Dynamical Systems}

A network consists of nodes and connecting edges between these nodes. Assuming that the topology, that is the connectivity pattern, of the network stays constant, the dynamics of a network are described by the intrinsic dynamics of each node and the dynamics of the coupling or interaction between nodes. Here we assume that the network is autonomous, it does not receive inputs from outside the network.

Generally the evolution of a time-continuous network is described by a set of equations of the following form:

$$
\dot{x}_{i}=f_{i}\left(x_{i}\right)+\sum_{j} I_{i, j}\left(x_{i}, x_{j}, t\right)
$$

with $i, j \in\{1, \ldots, N\}$. The function $f_{i}$ describes the intrinsic dynamics of node $i$. $I_{i, j}\left(x_{i}, x_{j}, t\right)$ captures the coupling or input from node $j$ to node $i$ at time $t$.

Intrinsic node dynamics. The intrinsic dynamics of each node can be very complex in itself. In this thesis we mainly consider phase oscillators, they are extremely simple while not settling into a steady state:

$$
\begin{aligned}
& \dot{x}=1 \\
& x\left(t^{-}\right)=1 \rightarrow x\left(t^{+}\right)=0 .
\end{aligned}
$$

The phase $x \in[0,1]$ proceeds with speed 1 , the phase $x=1$ is mapped onto $x=0$.

State dependent coupling. If the network is fully described by ODEs the dynamical equations become

$$
\dot{x}_{i}=f_{i}\left(x_{i}\right)+\sum_{j} g_{i, j}\left(x_{i}, x_{j}\right)
$$

$g_{i, j}$ is the coupling function from node $j$ and node $i$. The coupling depends on the current value of the dynamical variables $x_{j}$ and $x_{i}$. Another way of putting it is to say that the coupling is a function of the current state of the system. Therefore, we call this kind of coupling state dependent coupling.

Pulse-coupling. Other forms of coupling exist, in this thesis we focus on pulse coupling: In a pulse coupled system each time a specific event occurs in one node, it will 
send a pulse to the nodes it is connected to.

$$
\begin{aligned}
& \dot{x}_{i}=f_{i}\left(x_{i}\right)+Z_{i}\left(x_{i}\right) \sum_{j} \sum_{m} \varepsilon_{i, j} K_{i, j}\left(t-t_{j}^{m}\right) \\
& x_{j}\left(t_{j}^{m}\right)=x_{j}^{*} .
\end{aligned}
$$

The input to node $i$ is the sum of all incoming pulses send by all nodes $j$ of the network. The function $Z_{i}$ weighs the effect of the sum of all current inputs with the current state of node $i$. If the network consists of coupled phase oscillators $\left(f_{i}\left(x_{i}\right)=1\right)$, $Z_{i}$ is called phase response curve. $\varepsilon_{i, j}$ is the coupling strength from node $j$ to node i. $K_{i, j}(\tilde{t})$ is the coupling kernel with $K_{i, j}=0$ for $\tilde{t}<0$. The coupling kernel $K$ can have many shapes, including continuous and differentiable functions as well as $\delta$-functions. The set $\left\{t_{m} \mid m=1,2, \ldots\right\}$, is defined as all times when the value of $x_{j}$ was equal to the constant $x_{j}^{*}$, resulting in node $j$ sending a pulse. In this case the coupling does not (only) depend on the current state of the system, but on events that occurred in the past. Note that there exist different usages in the literature of the term 'pulse-coupling', that do not correspond to our definition, e.g. [44, 68].

\subsection{Important Theorems}

Existence and Uniqueness Theorem. The properties of the governing equations describing the dynamics of the systems provide restrictions on possible trajectories in phase space. In this thesis we will often consider a system of ODEs as reference system, to which we compare the dynamics of other systems. In a system described by smooth ODEs, the existence and uniqueness theorem holds (taken from 'Nonlinear Dynamics and Chaos' by Steven Strogatz, p.150 [82]):

Consider the initial value problem $\dot{\mathbf{x}}=\mathbf{f}(\mathbf{x}), \mathbf{x}(0)=\mathbf{x}_{\circ}$. Suppose that $\mathbf{f}$ is continuous and that all its partial derivatives $\partial f_{i} / \partial x_{j}, i, j=1, \ldots, n$, are continuous for $\mathbf{x}$ in some open connected set $D \subset \mathbb{R}^{n}$. Then for $\mathbf{x}_{\circ} \in D$, the initial value problem has a solution $\mathbf{x}(t)$ on some time interval $(-\tau, \tau)$ about $t=0$, and the solution is unique.

From this theorem it follows that as long as the function $\mathbf{f}$ is smooth enough locally, trajectories locally cannot intersect, which includes trajectories not joining or splitting in two. If the function $\mathbf{f}$ is globally smooth enough this holds everywhere.

Poincaré-Bendixson-Theorem. An important consequence of the existence and uniqueness theorem is the Poincaré-Bendixson-Theorem. It states that any bounded trajectory of a two dimensional smooth system eventually approaches a fixed point or limit cycle. A consequence of this theorem is that chaos is only possible in a smooth ODE system of at least three dimensions. 



\section{Chapter 3}

\section{Nested Limit Cycles in a Minimal Adaptive System}

\subsection{Introduction}

Network models in theoretical neuroscience often consist of adaptive units. What kind of dynamics can be expected of a minimal adaptive system? In this chapter we study the dynamics of a minimal adaptive system in greater detail. The phase space portrait is characterized by nested limit cycles. We provide a proof for the existence of limit cycles in a simplified system and finally consider the system from different perspectives.

Adaptation. What is adaptation? Adaptation is not a well defined term. However, there are systems that are generally considered to be adaptive. The evolution of species can be seen as a game of adaptation, different species competing over resources and fighting for survival. While species well adapted to their environment are likely to survive and flourish, other species that are less adapted are more likely to perish. A famous example is the evolution of the peppered moth, which has been documented for over two hundred years. This species of moth lives on light colored trees and lichens. While the moths were of light color originally, due to the increased pollution during the industrial revolution the trees darkened and consequently the typical moth found became darker, because a darker color better camouflaged against predators which increased likelihood for survival. Eventually the air became less polluted, the trees turned lighter again and the coloring of the typical moth returned to the original lighter color [93].

Another example of adaptation, this time from neuroscience, is the adaptation of neuronal firing rates [20, 23, 56. While a new stimulus elicits an increase in firing of neurons, the firing rate returns to baseline upon prolonged presentation of that stimulus. Adaptation is a general principle in the nervous system and occurs across processing levels and at a broad range of different time scales. An optical illusion stemming from adaptation is the motion after effect. If continuous motion in one direction is presented for a long time and the motion stimulus is then removed, observers report to perceive illusional motion in the opposing direction. While the motion stimulus was presented the neurons encoding this specific motion direction adapted to the stimulus by reducing their firing rate. Therefore, when the stimulus is removed, the neurons encoding the opposing direction of motion show higher firing rates than the adapted neurons. This disbalance in firing rates produces the percept of motion in the opposing direction [23, 56]. 
In both cases the adaptation can be understood as an optimization process. In evolution the phenotype of a species changes to optimize survival in a changing environment. In neuroscience the reduction of firing rates in response to a change in baseline inputs minimizes the production of energetically expensive action potentials.

Therefore, we choose the following working definition for adaptation:

Adaptation is an ongoing minimization of an energy functional, which itself may change on a slower time scale. Energy in this context does not refer to a physical energy but rather to a Lyapunov function, that is minimized along trajectories (except unstable points).

In theoretical neuroscience several networks and mechanisms were proposed to produce such dynamics, which Treves [87] termed latching dynamics. These networks are Hopfield-like networks [34], which are characterized by strongly connected cells assemblies competing with each other, resulting in several fixed points attractors. Hopfield-like networks can perform computational tasks, such as pattern completion, categorization and reproduce some perceptual effects such as priming or multistable perception [16, 66. If equipped with an additional mechanism, destabilizing attractors on a slower time scale latching dynamics can be observed: The system starts within the basin on an attractor and moves towards it. The additional mechanism destabilizes the current attractor basin and the system moves towards the next attractor. Overall the system produces a sequence of latching from one attractor to another. Mechanisms that were discussed for destabilizing attractors include synaptic or intraneural mechanisms, noise and inhibition [3, 39, 47, 48, 58, 66, 77, 87].

The adaptation process can be conceptualized as a particle - the adapting entitymoving inside a potential - the environment. With the above working definition of adaptation the simplest adaptive dynamical system consists of a one dimensional potential fully determining the movement of a particle, and a dynamical rule how the potential changes over time. We chose a sinusoidal potential which slowly changes according to the particle's position. The resulting system can be fully described by two ODEs, its phase space portrait is characterized by discrete limit cycles nested within each other.

Structure of this chapter. This chapter is structured in the following way: In the second section we introduce the system studied here. In the third section we demonstrate the system's behavior through numerical simulations. We explore different parameters and aim for an intuitive understanding of the system's behavior. While the original system studied is nonlinear and it is not possible to study it analytically we introduce a simplified system, that still qualitatively shows the same behavior but allows for some analytic treatment of global dynamics in a nonlinear dynamical system. In the fourth section we introduce this simplified system, show numerically that its qualitative behavior is the same and provide an analytic proof for the existence of the systems limit cycle behavior (at least for a certain parameter regime). In the fifth section we discuss the results by presenting alternative view points on the system. In the sixth and last section we provide a short summary. 


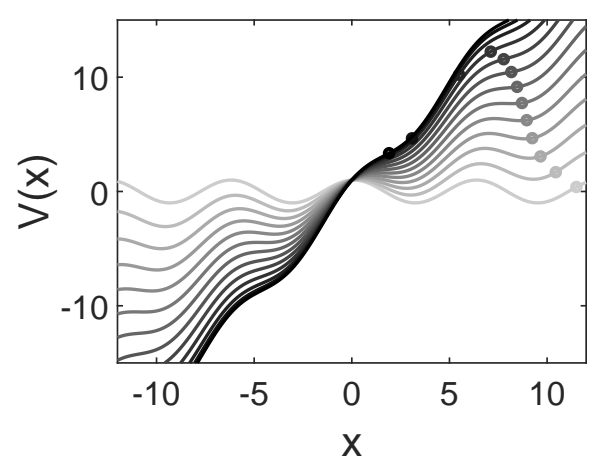

Figure 3.1: Particle interacting with its potential. The potential determines how the particle moves. At the same time the position of the particle leads to a change of the potential on a slower time scale. Lines correspond to $V(x(t))$, the dot $\circ$ corresponds to $(x(t), v(x(t))$ as time $t$ evolves. The lighter the color, the further in the past. Parameters: $A=1, \varphi=-\frac{\pi}{2}+0.1$, $\varepsilon=0.02, x_{\text {ini }}=11.5, c_{\text {ini }}=0$.

\subsection{The Original System}

\subsubsection{System Definition}

The adaptive system studied in this chapter is described by the following equations:

$$
\begin{aligned}
V(x, c) & =A \sin (x-\varphi)+c x \\
\dot{x} & =-\partial_{x} V \\
\dot{c} & =\varepsilon x
\end{aligned}
$$

$c, x \in \mathbb{R}$. The system has three parameters: the amplitude $A \geq 0$, the phase shift $\varphi$ and the time constant $\varepsilon . \varepsilon>0$ is rather small, ensuring that the environment changes slower than the adapting entity $x$.

The adaptive system can be conceptualized as a particle living inside a sinusoidal potential to which a straight line with slope $c$ is added. This slope changes depending on the position of the particle $x$. If $x$ is large the slope decreases and becomes more and more negative. If the position of the particle is in the negative region of the real space the opposite happens, the slope increases and becomes more and more positive. This leads the particle to move back and forth from one side to the other so that the overall dynamics resemble a seesaw (figure 3.1.

The above equations can be resolved into a simple system of only two ODEs:

$$
\begin{aligned}
\dot{x} & =-A \cos (x-\varphi)-c \\
\dot{c} & =\varepsilon x .
\end{aligned}
$$

There is another equivalent way of rewriting the system described by these two ODEs as a particle moving inside a potential. However, we will focus on the one presented above and leave the other for the appendix (section A). 


\subsubsection{A Numerical Study of the System's Behavior}

Figure 3.2 shows several simulated trajectories of the system for different parameters and initial conditions. The phase space portrait consists of several, potentially infinite many, equally spaced limit cycles nested within each other (panel A and B). Even though the shape of the cycles changes with $\varepsilon$, the topology of nested cycles stays intact (panel C). The behavior is robust against variations of other parameters (not shown). Only when $\varphi$ is taken to be $\pi$ (panel 4) or a multiple of $\pi$ the picture changes. Simulations do not yield a conclusive picture. Panel D shows three trajectories produced with different numerical integration algorithms while keeping parameters and initial condition identical. One trajectory spirals outwards, one inwards and one stays a cycle. While these numerical results indicate that the limit cycle behavior is rather robust it is not trivial to understand why it occurs. Figure 3.3 provides an intuition how to think about the system's behavior. But at the same time it is not clear why the system does not spiral in- or outwards. In the following part of the paper we will focus on this question and provide a proof for the limit cycle behavior in a simplified system.

\subsection{The Zigzag-Potential - a Simplified System}

\subsubsection{System Definition}

In the previous section we simulated a system displaying an intricate phase space structure of concentric limit cycles. However, we were not able to understand the limit cycle structure analytically. Here we present a simplified system that approximates the original system, shares the limit cycle structure in phase space and is simultaneously analytically tractable. The time evolution is defined via

$$
\begin{aligned}
\dot{x} & =(-1)^{\left\lfloor\frac{x-\varphi-\frac{\pi}{2}}{\pi}\right\rfloor} A-c \\
\dot{c} & =\varepsilon x
\end{aligned}
$$

The representation of a particle interacting with its potential leads to a zigzag potential, that is composed of segments of straight lines:

$$
\begin{aligned}
& V(x, c)=(-1)^{\left\lfloor\frac{x-\varphi-\frac{\pi}{2}}{\pi}\right\rfloor+1} A\left[x-\varphi-\left(\left\lfloor\frac{x-\varphi-\frac{\pi}{2}}{\pi}\right\rfloor+1\right) \pi\right]+c x \\
& \dot{x}=-\frac{\partial}{\partial x} V(x, c)
\end{aligned}
$$

Numerical simulations shows that the qualitative behavior of concentric limit cycles in phase space is conserved across the simplification (see figure 3.4 panel 1 and 2).

\subsubsection{Analytic Segment-Wise Solutions}

$\mathbf{x}$-segments and the sign $\mathbf{s}$. Now we consider $x$-segments:

$$
S_{l}:=\left\{x \mid\left\lfloor\frac{x-\varphi-\frac{\pi}{2}}{\pi}\right\rfloor=l\right\}
$$



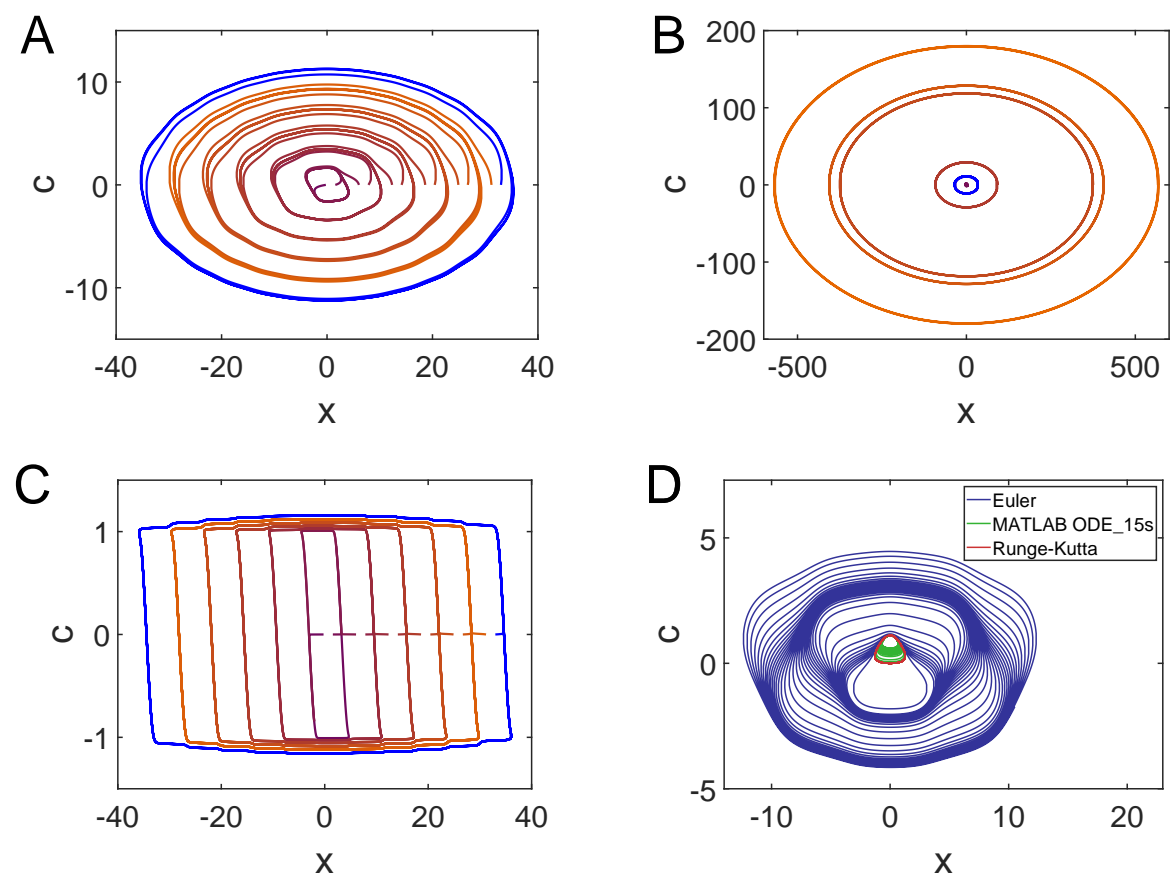

Figure 3.2: Trajectories of the original system in phase space. Panel A shows 12 trajectories starting from different initial particle positions. The phase space seems to consist of stable and unstable limit cycles nested within each other. Panel B shows 6 trajectories in phase space. Identical parameters are used as in panel A. Even for large initial particle positions the limit cycle structure still seems to exist. The blue trajectories in panel $\mathrm{A}$ and $\mathrm{B}$ are identical. Panel $\mathrm{C}$ again shows trajectories in phase space. Here $\varepsilon$ was chosen to be small. The trajectories become more square shaped. The initial conditions are identical to the ones used in panel A. Panel D shows a single trajectory in phase space simulated with three different numerical integration algorithms for a system with $\varphi=\pi$. While all three graphs start from the same initial condition, the trajectory simulated with a simple euler algorithm circles outwards (blue), using a preimplemented algorithm for fast-slow systems of Matlab produces an inward spiraling trajectory (green). Finally using a Runge-Kutta algorithm 4th order produces a cycle (red). Parameters: $A=1, c_{\text {ini }}=0$. Panel A: $\varepsilon=0.1, \varphi=-\frac{\pi}{2}+0.1, t \in[0,80], x_{\text {ini }} \in\left\{a \pi-\frac{\pi}{2}, a \pi-\frac{\pi}{2}-1.8\right\}$ with $a \in\{1,3,5,7,9,11\}$; Panel B: $\varepsilon=0.1, \varphi=-\frac{\pi}{2}+0.1, t \in[0,10000]$, $x_{\text {ini }} \in\left\{a \pi-\frac{\pi}{2}\right\}$ with $a \in\{1,11,30,120,130,181\}$; Panel C: Parameters as in panel A except for $\varepsilon=0.0001$ and $t \in[0,10000]$; Panel D: $\varepsilon=0.11$, $\varphi=\pi, t \in[0,1000], x_{\text {ini }}=0.01$ 

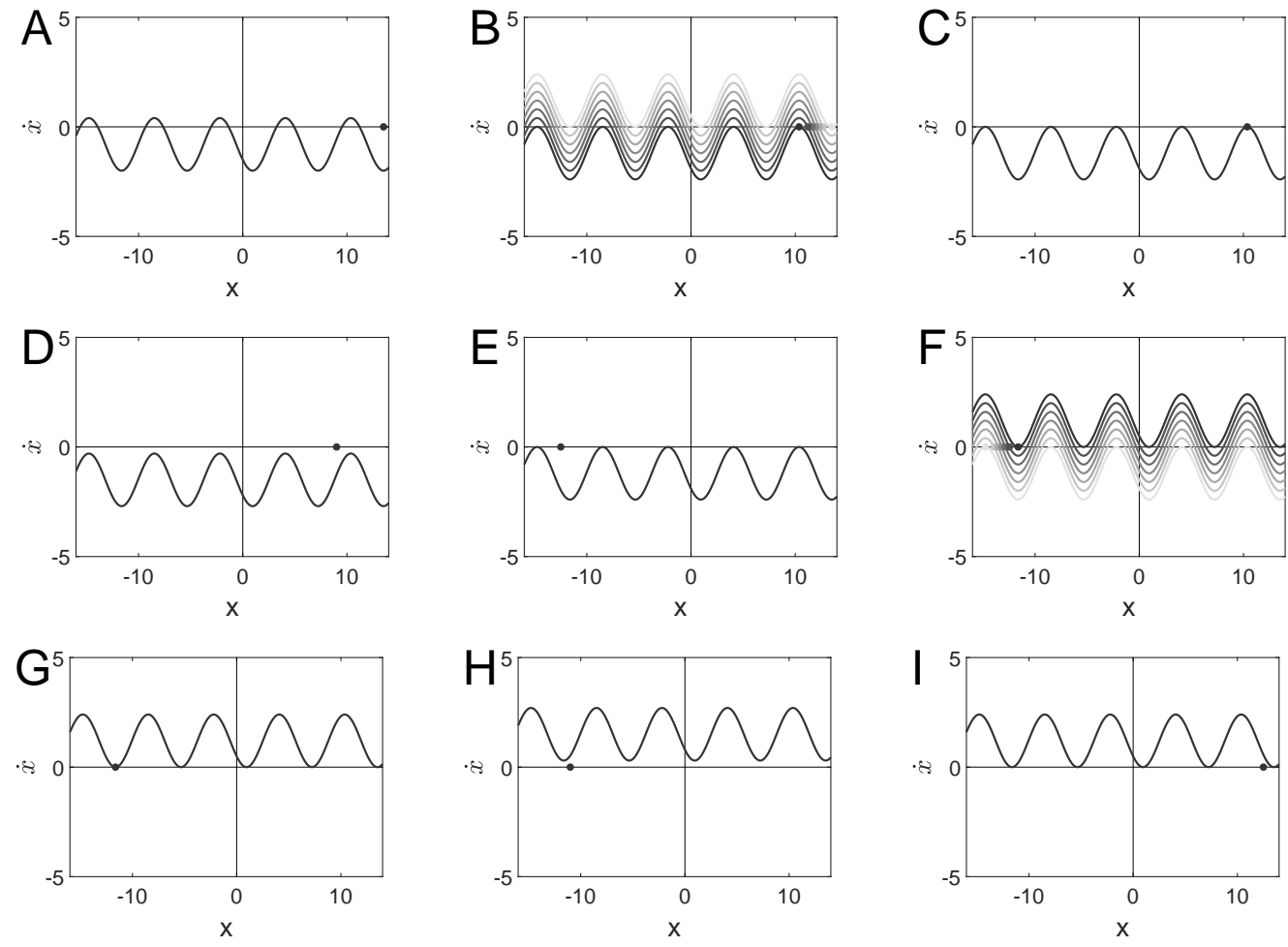

Figure 3.3: Intuition for the limit cycle behavior in the case of $\varepsilon \ll 1$. Depicted is a series of events within a full cycle, each panel shows how the velocity $\dot{x}$ depends on the particle's position $x$. The other dynamic variable $c$ is captured by the shift of the cosine wave with positive $\mathrm{c}$ corresponding to a shift downwards and vice versa. In panel A the particle starts from an arbitrary initial condition, $c$ is taken to be zero. Because $\varepsilon$ is small the movement of the cosine wave will be slow compared to the movement of the particle. Hence the particle moves fast towards the zero crossing of the curve. It then tracks the zero crossing closely as the curve slowly moves down (panel B). At some point the curve looses touch with the zero-line (panel $\mathrm{C}$ ), the particle is free to quickly move over to the other side (panel D). Once the particle passes $x=0$ the curve starts moving up again. Hence at some point the curve is touching the zero line (panel $\mathrm{E})$, the particle moves to the respective zero crossing and closely tracks its position while the curve continues moving upwards (panel F). In panel $\mathrm{G}$ the curve looses touch again, the particle is then free to quickly move over to the other side (panel $\mathrm{H}$ ) until the curve touches the zero line again (panel I) and the cycle starts anew. 

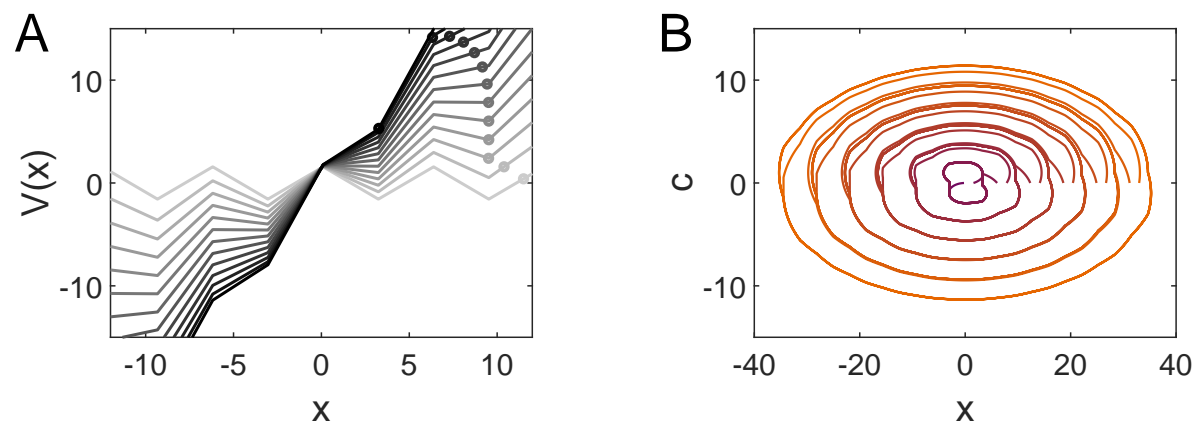

Figure 3.4: Potential and trajectories in phase space of the simplified system. Panel A and B are constructed analog to figure 3.1 and figure 3.2 , panel A. Identical parameters are used. The zigzag behavior arising through the interaction between the particle and its potential is visible in panel A. Panel B shows a phase space structure of nested limit cycles very similar to the original system.

Within each segment the sign $s(x):=(-1)^{\left\lfloor\frac{x-\varphi-\frac{\pi}{2}}{\pi}\right\rfloor}$ is either fixed at $=1$ or $=-1$.

Solving for $\mathbf{c}(\mathbf{x})$. For each segment $S_{l}$ the differential of $c$ with respect to $x$ can be solved:

$$
\frac{\mathrm{d} c}{\mathrm{~d} x}=\frac{\frac{\mathrm{d} c}{\mathrm{~d} t}}{\frac{\mathrm{d} x}{\mathrm{~d} t}}=\frac{\varepsilon x}{s A-c}
$$

By separation of variables we obtain:

$$
\begin{aligned}
c(x) & =s A \pm \sqrt{A^{2}-\left(\varepsilon x^{2}+C\right)} \\
C & =-\varepsilon x_{\circ}^{2}-c_{\circ}^{2}+2 s c_{\circ} A
\end{aligned}
$$

with $x_{\circ}, c_{\circ} \in S_{l}$ as initial conditions such that $x\left(t_{\circ}\right)=x_{\circ}$ and $c\left(t_{\circ}\right)=c_{\circ}$. The choice whether the root term needs to be added or subtracted has to be taken such that $\left(x_{\circ}, c_{\circ}\right)$ is part of the solution $c(x)$. These equations can be rewritten as:

$$
c\left(x_{\circ}+\Delta x\right)=s A \pm \sqrt{\left(A-s c_{\circ}\right)^{2}-\varepsilon \Delta x\left(2 x_{\circ}+\Delta x\right)}
$$

with $\Delta x=x-x_{\circ}$.

Solutions correspond to ellipse segments The solution $c(x)$ is an elliptic equation. It can be rewritten as ellipse normal form $\left(\frac{x^{2}}{a^{2}}+\frac{y^{2}}{b^{2}}=1\right)$ with the following substitution:

$$
\begin{aligned}
y & =c(x)-s A \\
b & =\sqrt{A^{2}+\varepsilon x_{\circ}^{2}+c_{\circ}^{2}-2 s c_{\circ} A} \\
a & =\sqrt{\frac{b^{2}}{\varepsilon}} .
\end{aligned}
$$



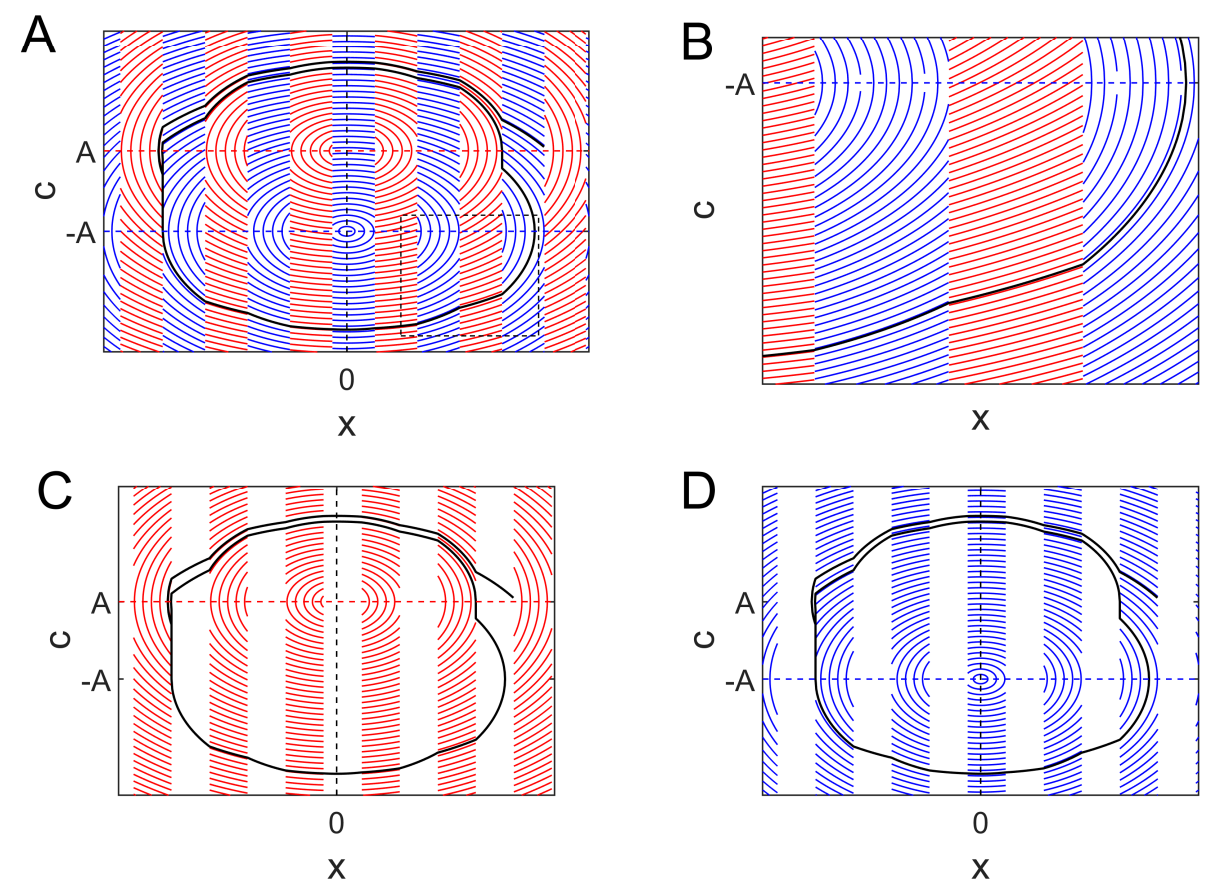

Figure 3.5: A trajectory closely follows elliptic segments. The segments are shifted up by $A$ if $x \in S \uparrow$ depicted in red and down if $x \in S \downarrow$ depicted in blue. The trajectory is analytically calculated segment vise using the border point of one segment as initial condition for the next segment. Panel B as an enlarged part of panel A shows the continuous trajectory bending at segment borders and following the ellipses closely. Panel $\mathrm{C}$ and D show only the elliptic segments of $S^{\uparrow}$ and $S^{\downarrow}$, respectively. The elliptic nature becomes visible. Parameters: $A=1, \varepsilon=0.4, \varphi=0.5$.

Figure 3.5 shows some example trajectories for each segment. Segments alternatingly produce trajectories which belong to ellipses centered either around $(0, A)$ (depicted in red) or $(0,-A)$ (depicted in blue).

Up and down shifted segments. We will call these segments upshifted and downshifted segments, respectively:

$$
\begin{aligned}
& S^{\uparrow}=\{x \in \mathbb{R} \mid s(x)=1\} \\
& S^{\downarrow}=\{x \in \mathbb{R} \mid s(x)=-1\} .
\end{aligned}
$$

A piece-wise smooth system. A trajectory $(c(t), x(t))$ proceeds in counter clockwise direction in phase space. For the proof section we are not interested in the time dependence, but only in how the two dependent variables $c$ and $x$ relate to each other. We have seen above, that within a segment a dependence between both coordinates can be derived: $c(x)$.

A trajectory passes over segment borders. Each time a trajectory crosses such a border, the sign $s \in\{-1,1\}$ switches, depending on whether the next segment is up 
or down shifted. Since trajectories are continuous this leads to a non-smooth kink in the trajectory at segment borders. Also the time independent curve $c(x)$ is continuous and has bents at segment borders. The system falls into the class of piece-wise smooth systems [10]. Further the system is a commutable pendulum (see reference [65] for another example). To analytically calculate the full curve $c(x)$, the solutions for $c(x)$ need to be determined segment-wise, going in counter clockwise direction and switching the sign $s$ when passing a segment border. The sign before the root term switches when passing $c=-A$ in a down shifted segment or $c=+A$ in an up shifted segment. Note that the curve $c(x)$ is not necessarily a function, since there are several $c$-values corresponding to one $x$-value originating from the same trajectory.

Remarks on monotonicity. $\quad c(x)$ in any up shifted segment is strictly monotonically increasing with $x$ if $x<0$ and $c>A$ or $x>0$ and $c>A$. Otherwise $c(x)$ is strictly monotonically decreasing. $c(x)$ in any down shifted segment is strictly monotonically increasing with $x$ if $x<0$ and $c>-A$ or $x>0$ and $c<A$. Otherwise $c(x)$ is strictly monotonically decreasing. It follows that any connected part of $c(x)$ increases strictly monotonically with $x$ while $c>A$ and $x<0$. Also any connected part of $c(x)$ increases strictly monotonically with $x$ while $c<-A$ and $x>0$. Any connected part of $c(x)$ decreases strictly monotonically with $x$ while $c<-A$ and $x<0$ and also while $c>A$ and $x>0$.

\subsubsection{Proof for Finite Time Convergence to Limit Cycles - Core Ideas}

Numerical simulations show concentric limit cycles in phase space. Trajectories within their basin of attraction reach these cycles in finite time. In this and the following section we provide a proof for this behavior. Each of these sections covers the full proof and is complete in itself. This first section aims at an intuitive understanding to provide the reader with a quick way to grasp the core ideas using graphical material. The next second section aims at providing a rigorous proof covering details that are not covered in the first. The structures of both sections run in parallel allowing the reader to easily change between both modes of presentation.

The proof consists of several parts. In the first part a region in phase space is defined that contains a structure we denote funnel structure. All trajectories entering this structure merge in finite time into a discrete set of trajectories leaving the structure through predefined exit points. In the second part we show that the trajectory leaving a specific exit point will eventually reach another corresponding exit point. And the trajectory leaving this exit point eventually reaches the original exit point. Therefore, cycles exist.

\section{Finite Time Convergence within a Funnel Structure}

Region with funnel structure. The horizontal axis of the elliptic curve segments $c(x)$ belonging to the up shifted segments $S^{\uparrow}$ is situated at $c=A$. The horizontal axis of the elliptic curve segments $c(x)$ belonging to the down shifted segments $S^{\downarrow}$ is situated at $c=-A$. Hence, above $c=A$ all curve segments $c(x)$, independent of segment type, are situated above the horizontal elliptic axis and belong to the upper half of an ellipse, below $c=-A$ all curve segments $c(x)$, independent of segment 

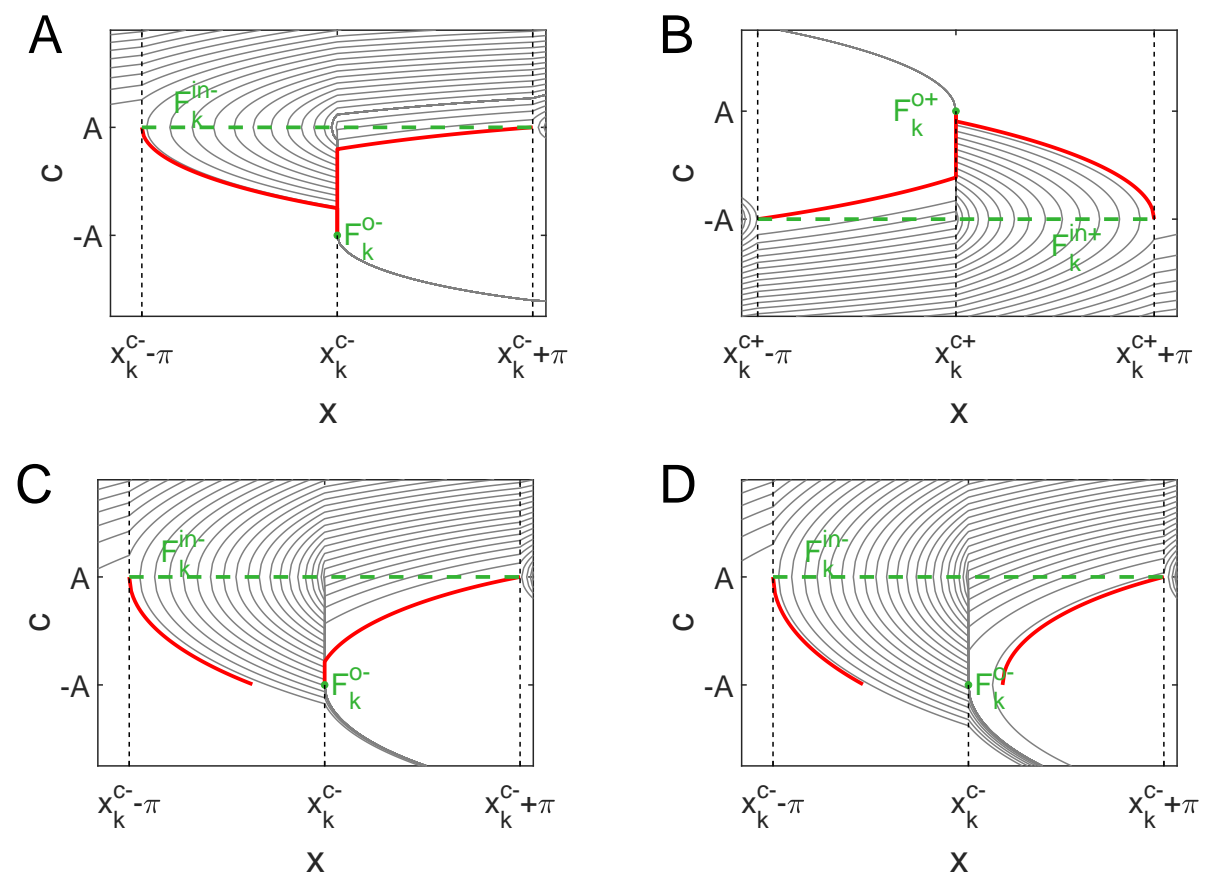

Figure 3.6: Examples for funnels $\mathbf{F}_{\mathbf{k}}^{+}$and $\mathbf{F}_{\mathbf{k}}^{-}$as well as examples for open and closed funnels. Panel $\mathrm{A}$ shows an example of a funnel situated at $x<0$, the funnel exit point $F_{k}^{\mathrm{o}-}$ at $x=x_{k}^{\mathrm{c}-}$ and $c=-A$ and the funnel entrance region $F_{k}^{\mathrm{in}-}$ with $x \in\left[x_{k}^{\mathrm{c}-}-\pi, x_{k}^{\mathrm{c}-}+\pi\right)$ and $c=A$. The funnel is closed because the two trajectories passing through the funnel borders reach the funnel center $x_{k}^{\mathrm{c}-}$ before reaching $c=-A$. Hence, all trajectories entering through the input region $F_{k}^{\text {in- }}$ leave the funnel as a single trajectory passing through the funnel exit point $F_{k}^{o-}$. Panel B shows an example for a closed funnel $F_{k}^{+}$situated at $x>0$. Again funnel input region $F_{k}^{\text {in }+}$ and funnel exit point $F_{k}^{o+}$ and the two bounding trajectories are shown. Panel $\mathrm{C}$ and $\mathrm{D}$ show examples of open funnels. In $\mathrm{C}$ the left bounding trajectory does not reach the funnel center $x_{k}^{\mathrm{c}-}+\pi$ before reaching $c=-A$. Not all trajectories leave the funnel at the funnel exit point $F_{k}^{\mathrm{o}-}$. In panel $\mathrm{D}$ neither bounding trajectory reaches the funnel center before reaching $c=-A$. Many trajectories leave the funnel not at the funnel exit point. Parameters: $A=1, \varphi=0.5, \varepsilon=0.04$ (Panel A,B), $\varepsilon=0.105$ (Panel C), $\varepsilon=0.14$ (Panel D). 
type, are situated below the horizontal elliptic axis and belong to the lower half of an ellipse. But in the region $-A<c<A$ the situation is mixed (see figure 3.5 . panel A). Within down shifted segments $\left(x \in S^{\downarrow}\right)$ the curve segments $c(x)$ lie above the horizontal elliptic axis, within up shifted segments $\left(x \in S^{\uparrow}\right)$ the curve segments $c(x)$ lie below the horizontal elliptic axis. This gives rise to a structure consisting of elements we call funnels. A funnel has the dynamic property of collapsing a set of trajectories onto a single trajectory. This property will be explained further below (see figure 3.6).

Funnel center, funnel entrance and funnel exit point. Each funnel $F_{k}$ has a funnel center $x_{k}^{\mathrm{c} \pm}$, with $k \in\{1,2 \ldots\}$ denoting funnel identity. $x_{k}^{\mathrm{c}+}$ corresponds to the $k$ th funnel center on the right $(x>0)$ and $x_{k}^{\mathrm{c}-}$ to the $k$ th funnel center on the left $(x<0)$, respectively. The point $\left(x=x_{k}^{c} \pm, c= \pm A\right)=F_{k}^{o \pm}$ is the funnel output or exit point. Further, each funnel has an input region $F_{k}^{\text {in } \pm}=\left\{(x, c) \mid x \in\left[x_{k}^{\mathrm{c} \pm}-\pi, x_{k}^{\mathrm{c} \pm}+\pi\right) \wedge c=\right.$ $\mp A\} . x_{k}^{\mathrm{c}}$ are chosen such that each funnel entrance fully lies within $x<0$ or fully lies within $x>0$.

The flow within a funnel. Within a funnel the flow in $x$-direction goes towards the funnel center $x_{k}^{c}$, while for $x<0$ the flow in $c$-direction moves down $(\dot{c}<0)$ and for $x>0$ the flow in $c$-direction moves up $(\dot{c}>0)$. Hence all trajectories passing through the funnel entrance will eventually pass through $c=-A$ for $x<0$ and $c=A$ for $x>0$. They are bounded by the trajectories passing through the funnel entrance borders $(x, c)=\left(x_{k}^{\mathrm{c}}-\pi, \pm A\right)$ and $(x, c)=\left(x_{k}^{\mathrm{c}}+\pi, \pm A\right)$.

Open and closed funnels. If these two trajectories reach the funnel center $x_{k}^{\mathrm{c}}$ before they reach $c= \pm A$ for $x \gtrless 0$ they will leave the funnel at the funnel exit point $F_{k}^{\mathrm{o} \pm}$ and so will all other trajectories entering the funnel at $F_{k}^{\text {in } \pm}$ between the two bounding trajectories. In this case the funnel is closed, all trajectories entering the funnel converge in finite time onto a single trajectory passing through $F_{k}^{o \pm}$ (see figure 3.6. panels $\mathrm{A}$ and $\mathrm{B})$.

If one (figure 3.6, panel C) or both (figure 3.6, panel D) bounding trajectories reach $x= \pm A$ (for $x \gtrless 0$ ) before reaching the funnel center $x_{k}^{\mathrm{c}}$ there is a set of trajectories leaving the funnel not at the funnel exit point $F_{k}^{o \pm}$. The funnel is open. The condition that both bounding trajectories need to reach the funnel center $x_{k}^{c}$ before reaching $c= \pm A$ provides parameter constraints for a specific funnel $F_{k}^{ \pm}$to be closed:

$$
\frac{4 A^{2}}{\pi\left(2\left|x_{k}^{\mathrm{c}}\right|+\pi\right)}>\varepsilon_{k, c}^{\mathrm{o}}
$$

Short recapitulation. We set out to show that there exists a region in phase space with trajectories converging in finite time onto limit cycles. In this section 3.3 .3 we have characterized a structure in phase space within $-A<c<A$ we call funnel. If a funnel is closed all trajectories passing through the funnel entrance region $F_{k}^{\text {in }} \pm$ converge onto a single trajectory passing through $F_{k}^{\mathrm{o \pm}}$ in finite time. However, we still have to show that this single trajectory is a cycle. A trajectory is a cycle if a point of the trajectory is visited again. 


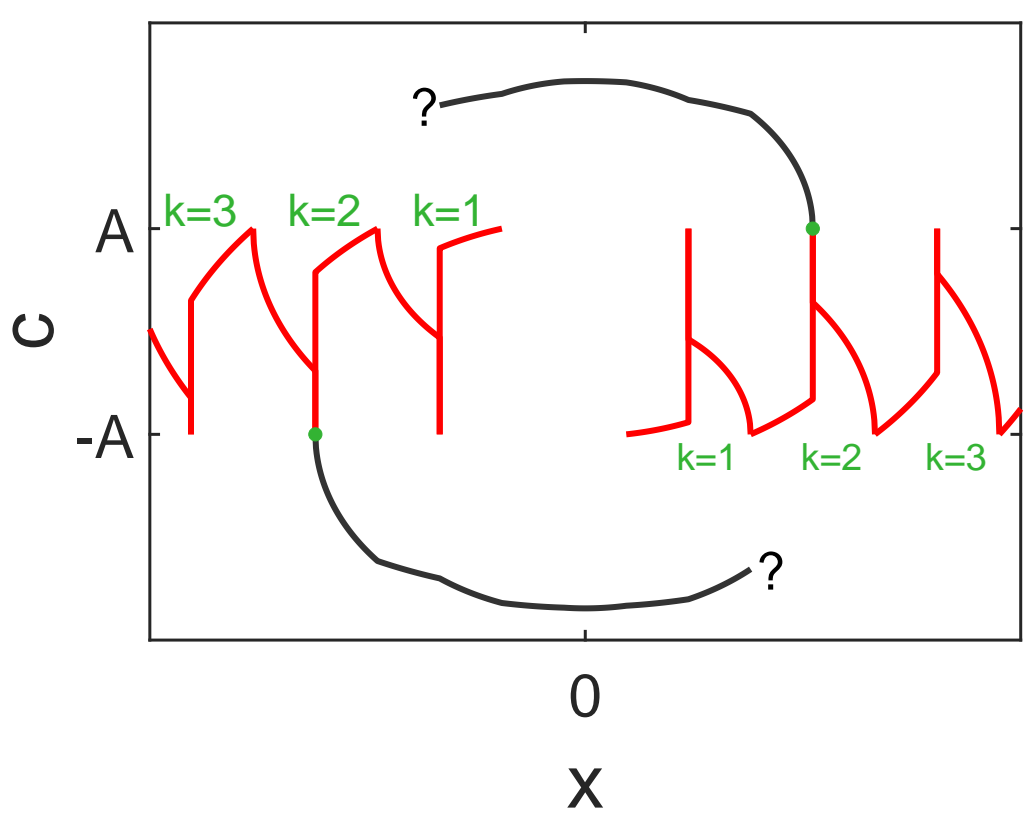

Figure 3.7: How do funnels connect? A trajectory leaving at a funnel exit point will eventually reach the funnel entrance region at the opposing side. However, it is not clear at which $x$-position. Subsection (ii) is concerned with showing that a trajectory leaving a specific funnel $k$ at the funnel exit point $F_{k}^{o \pm}$ will reach the funnel entrance region of the corresponding $k$ th

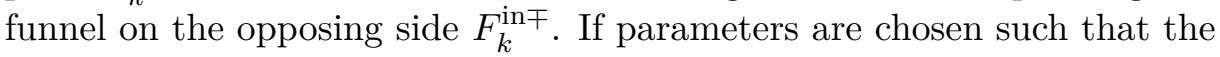
funnels are closed it follows that the trajectory will be a cycle and not a spiral. Parameters: $A=1, \varepsilon=0.02, \varphi=0.5$.

\section{Existence of Limit Cycles}

Proving the existence of cycles only requires to show how funnels connect. Let us assume that the parameters are chosen such that the funnel $F_{k}^{-}$and $F_{k}^{+}$both are closed. We will show that a trajectory passing through $F_{k}^{\text {o+ }}$ will eventually reach this point again. Specifically we will show that this trajectory passes sequentially through the following states:

$$
F_{k}^{\mathrm{o}+} \stackrel{(i i)}{\longrightarrow} F_{k}^{\mathrm{in}-} \stackrel{(i)}{\longrightarrow} F_{k}^{\mathrm{o}-} \stackrel{(*)}{\longrightarrow} F_{k}^{\mathrm{in}+} \stackrel{(i)}{\longrightarrow} F_{k}^{\mathrm{o}+}
$$

The validity of the state transitions $(i)$ directly follows from the assumption that both funnels $F_{k}^{ \pm}$are closed (see section 3.3.3. The transition $(*)$ is fully analog to the transition (ii) and included in the appendix (section C). Hence only the transition (ii) remains to be proven, it remains to show that a trajectory leaving funnel $k$ on the right side reaches the funnel entrance region of funnel $k$ on the left side (see figure 3.7).

$\boldsymbol{x}_{k}^{*-}$ - the position where the trajectory returns to $\boldsymbol{c}=\boldsymbol{A} . x_{k}^{*-}$ is defined as the $x$-value of the trajectory leaving $F_{k}^{\mathrm{o}-}$ when it reaches $c=A$ again on the left 


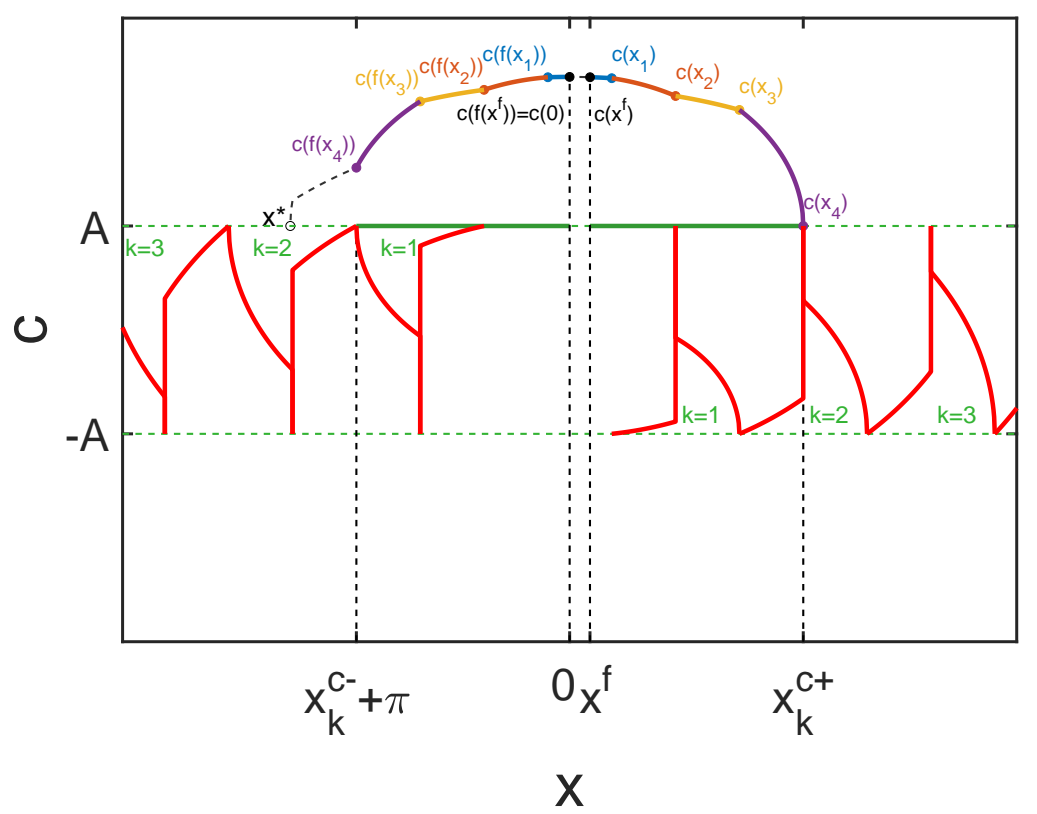

Figure 3.8: $\mathbf{A}$ trajectory leaving $\mathbf{F}_{\mathbf{k}}^{+}$will reach to the left of the right funnel entrance of $\mathbf{F}_{\mathbf{k}}^{-}$. An induction argument shows that the trajectory leaving at the funnel exit $F_{k}^{\mathrm{o}+}$ reaches $c=A$ again to the left of the right funnel entrance border of funnel $F_{k}^{-}$(ii.1). First, $x^{\mathrm{f}}>0$ and a mapping $f(x)$ are defined. $x^{\mathrm{f}}$ is defined such that the distance in $x$-direction of the funnel exit point $F_{k}^{\mathrm{o}+}$ to $x^{\mathrm{f}}$ is identical to the distance between 0 and the right funnel entrance border $x_{k}^{\mathrm{c}-}+\pi$, compare the two solid green lines. Second it is shown that $c\left(x^{\mathrm{f}}\right)<c\left(f\left(x^{\mathrm{f}}\right)\right)$ (depicted as black dots). Third, it is then shown that this inequality continuous to hold when increasing $x$. Dots are plotted at segment borders. The dots positioned at $x$ and corresponding $f(x)$ are depicted with the same color. The dots on the left side lie above their equicolored counterparts on the right. Importantly, this is also true for the dot situated at the funnel entrance border $x_{k}^{\mathrm{c}-}+\pi$ compared to the dot at the funnel exit point $F_{k}^{\mathrm{o}+}$. Because the curve $c(x)$ is monotonically increasing in $x$ for $x<0$ and $c>A$ the point $x^{*}$ where the curve reaches $c=A$ has to be situated to the left of the right funnel entrance border $x_{k}^{\mathrm{c}-}+\pi$. Parameters: $A=1, \varepsilon=0.02, \varphi=0.5$. 


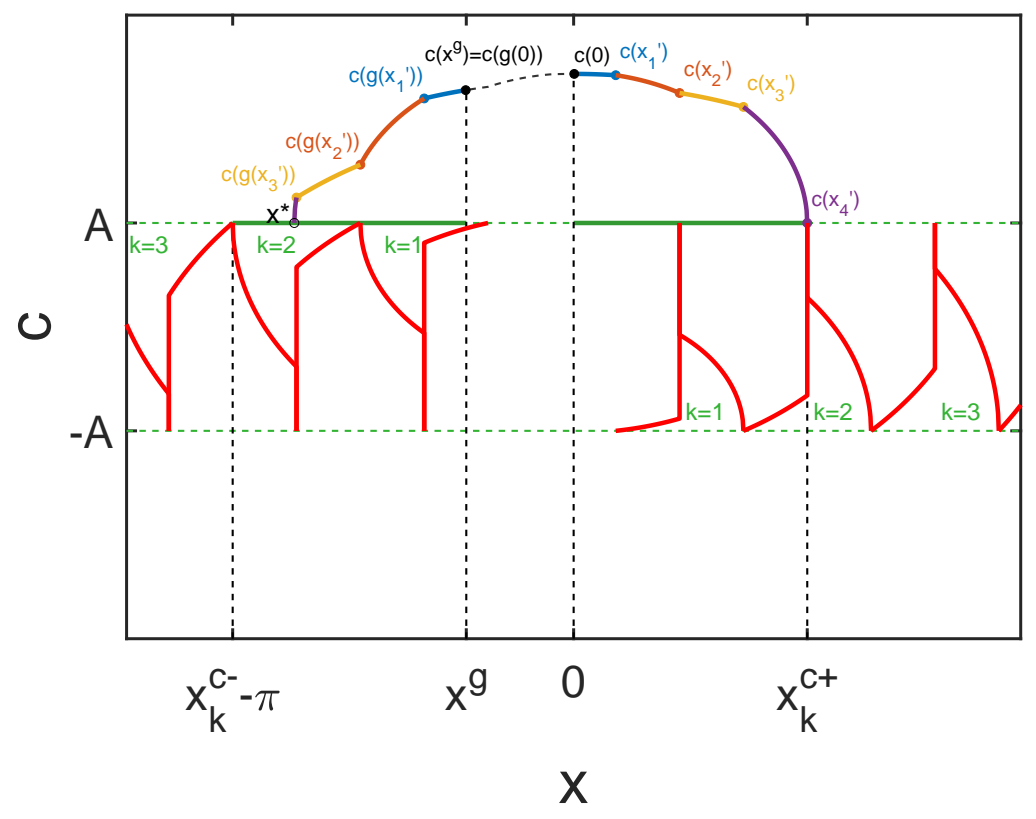

Figure 3.9: A trajectory leaving $\mathbf{F}_{\mathbf{k}}^{+}$will reach to the right of the left funnel entrance of $\mathbf{F}_{\mathrm{k}}^{-}$. A second induction argument shows that the trajectory leaving at the funnel exit $F_{k}^{\mathrm{o}+}$ reaches $c=A$ again to the right of the left funnel entrance border of funnel $F_{k}^{-}$(ii.2). First, $x^{\mathrm{g}}<0$ and a mapping $g(x)$ are defined. $x^{\mathrm{g}}$ is defined such that the distance in $x$-direction of the funnel exit point $F_{k}^{\mathrm{o}+}$ to 0 is identical to the distance between $x^{\mathrm{g}}$ and the left funnel entrance border $x_{k}^{\mathrm{c}-}-\pi$, compare the two solid green lines. Second it is shown that $c\left(x^{\mathrm{g}}\right)=c(g(0))<c(0)$ (depicted as black dots). Third, it is then shown that this inequality continuous to hold when increasing $x$. Dots are plotted at segment borders. The dots positioned at $x$ and corresponding $g(x)$ are depicted with the same color. The dots on the left side lie below their equicolored counterparts on the right. It also follows that the point with $x>0$ corresponding to $x^{*}$ lies above $c=A$. Because $c(x)$ is monotonically decreasing for $c>A$ and $x>0$ the funnel exit point lies further outwards relative to this point. The same is true for the corresponding left funnel border of $F_{k}^{-}$, which lies further outward relative to $x^{*}, x^{*}>x_{k}^{\mathrm{c}-}-\pi$. Parameters: $A=1, \varepsilon=0.02, \varphi=0.5$. 
side $(x<0)$. To show that the trajectory reaches the funnel entrance of funnel $k$ $\left(\left(x_{k}^{*-}, A\right) \in F_{k}^{\text {in- }}\right)$, we will first show that $x_{k}^{*-}$ lies to the left of the right funnel entrance border $\left(x_{k}^{*-}<x_{k}^{\mathrm{c}-}+\pi\right)$ (ii.1, see figure 3.8) and in a second part show that $x_{k}^{*-}$ lies to the right of the left funnel entrance border $\left(x_{k}^{\mathrm{c}-}-\pi \leq x_{k}^{*-}\right)$, (ii.2, see figure 3.9 .

(ii.1) To Show: $x_{k}^{*-}<x_{k}^{\mathrm{c}-}+\pi$; $x_{k}^{*-}$ Lies Left of the Right Entrance of $F_{k}^{\text {in- }}$

Defining $x^{\mathrm{f}}, f(x)$, replacing the trajectory leaving at $F_{k}^{\mathrm{o}+}$ by the function $c(x)$. $x^{\mathrm{f}}>0$ is defined as the $x$-value that has the same distance from the right funnel center $x_{k}^{\mathrm{c}+}$ as the right funnel entrance border from $x=0: 0-\left(x_{k}^{\mathrm{c}-}+\pi\right)=x_{k}^{\mathrm{c}+}-x^{\mathrm{f}}$. Further, $f(x)=-x+x^{\mathrm{f}}$. It can be shown that $x$ and $f(x)$ always lie within the same segment type, either $x$ and $f(x) \in S^{\uparrow}$ or $x$ and $f(x) \in S^{\downarrow} ; s(x)=s(f(x))$. In the paragraph 3.3.2, 'remarks on monotonicity' it was discussed that any connected curve $c(x)$ with $c>A$ is strictly monotonically decreasing with $x$ while $x>0$ and strictly monotonically increasing while $x<0$. Here we study the trajectory $(x(t), c(t))$ from leaving a funnel exit point until reentering another funnel, hence, $c>A$ and the corresponding curve $c(x)$ is a function, there is exactly one $c$-value assigned to any $x$-value.

Induction. to show: $c\left(f\left(x_{k}^{c+}\right)\right)=c\left(x_{k}^{c-} \pi\right)>c\left(x_{k}^{x+}\right)=A$

Induction step. to show: $c(f(x))>c(x) \Longrightarrow c(f(x+\Delta x))>c(x+\Delta x)$ with $\Delta x>0$.

First, consider $\Delta x$ such that segment borders are not crossed and define $x_{i+1}=$ $x_{i}+\Delta x$.

$$
\begin{aligned}
c\left(f\left(x_{i+1}\right)\right)=c\left(-x_{i+1}+x^{f}\right)=c\left(-x_{i}-\Delta x+x^{f}\right)=c\left(f\left(x_{i}\right)+(-\Delta x)\right) & c\left(x_{i+1}\right)=c\left(x_{i}+\Delta x\right) \\
& \Longleftrightarrow s\left(f\left(x_{i}\right)\right) A+\sqrt{\left(A-s\left(f\left(x_{i}\right)\right) c\left(f\left(x_{i}\right)\right)\right)^{2}-\varepsilon(-\Delta x)\left(2 f\left(x_{i}\right)+(-\Delta x)\right)} \\
& >s\left(x_{i}\right) A+\sqrt{\left(A-s\left(x_{i}\right) c\left(x_{i}\right)\right)^{2}-\varepsilon(\Delta x)\left(2 x_{i}+\Delta x\right)} \\
\Longleftrightarrow & \left(A-s\left(x_{i}\right) c\left(f\left(x_{i}\right)\right)\right)^{2}-\varepsilon \Delta x\left(-2\left(-x_{i}+x^{f}\right)+\Delta x\right) \\
& >\left(A-s\left(x_{i}\right) c\left(x_{i}\right)\right)^{2}-\varepsilon \Delta x\left(2 x_{i}+\Delta x\right) \\
\Longleftrightarrow & \left(A-s\left(x_{i}\right) c\left(f\left(x_{i}\right)\right)\right)^{2}+2 \varepsilon \Delta x x^{f}>\left(A-s\left(x_{i}\right) c\left(x_{i}\right)\right)^{2}
\end{aligned}
$$

The last inequality holds because we assumed $c\left(f\left(x_{i}\right)\right)>c\left(x_{i}\right)$ and $c \geq A>0$ and $s(x)=s(f(x))$ holds. So far only $\Delta x$ were considered, such that $x$ and $x+\Delta x$ are situated within the same segment. However the argument also holds for $\Delta x$ that induce a shift across segment borders: In that case $\Delta x$ can be rewritten as $\Delta x=\sum_{i=1}^{n} \Delta x_{i}$ and the above argument can be applied sequentially passing from segment border to segment border: We choose $\Delta x_{i}$, such that for each $l$ the $x$-value $x+\sum_{i=1}^{l} \Delta x_{i}$ 
corresponds to a segment border. $c\left(f\left(x+\sum_{i=1}^{l} \Delta x_{i}\right)\right)>c\left(x+\sum_{i=1}^{l} \Delta x_{i}\right) \Longrightarrow$ $c\left(f\left(x+\sum_{i=1}^{l+1} \Delta x_{i}\right)\right)>c\left(x+\sum_{i=1}^{l+1} \Delta x_{i}\right)$.

Induction start. to show: $c\left(f\left(x^{f}\right)\right)=c(0)>c\left(x^{f}\right)$

This follows from the observation that $c(x)$ decreases monotonically with $x$ for $c>A$ and $x>0$.

Conclusion. It follows that $c\left(x_{k}^{\mathrm{c}-}+\pi\right)=c\left(f\left(x_{k}^{\mathrm{c}+}\right)\right)>c\left(x_{k}^{\mathrm{c}+}\right)=A$. Therefore, when the trajectory passes the right funnel entrance border of funnel $F_{k}^{-}$, the trajectory does not reach the funnel entrance but lies above it. Because $c(x)$ is monotonically increasing with $x$ for $c>A$ and $x<0$, the point $x^{*}$ when the trajectory reaches the funnel entrance $\left(c\left(x_{k}^{*-}\right)=A\right)$ has to lie to the left of the right funnel entrance: $x_{k}^{*-}<x_{k}^{\mathrm{c}-}+\pi$.

\section{(ii.2) To Show: $\mathrm{x}_{\mathrm{k}}^{\mathrm{c}-}+\pi \leq \mathrm{x}^{*} ; x_{k}^{*-}$ Lies Right of the Left Entrance of $F_{k}^{\mathrm{in}-}$}

Defining $\boldsymbol{x}^{\mathrm{g}}, \boldsymbol{g}(\boldsymbol{x}) . \quad x^{\mathrm{g}}<0$ is defined as the $x$-value that has same distance to the left border of the funnel entrance $F_{k}^{\text {in- }}$ as the funnel exit point $F_{k}^{\mathrm{o}+}$ from $x=0$ : $x_{\mathrm{g}}-\left(x_{k}^{\mathrm{c}-}-\pi\right)=x_{k}^{\mathrm{c}+}-0$. Further, $g(x)=-x+x^{\mathrm{g}}$. It can be shown that $x$ and $g(x)$ always lie within the same segment type, either $x$ and $g(x) \in S^{\uparrow}$ or $x$ and $g(x) \in S^{\downarrow}$; $s(x)=s(g(x))$.

Induction. to show: When increasing $x, c\left(g\left(x^{\prime}\right)\right)=c\left(x_{k}^{*-}\right)=A$ occurs before $c\left(x_{k}^{c+}\right)=$ $A$ and hence $g\left(x_{k}^{c+}\right)=x_{k}^{c-}-\pi<g\left(x^{\prime}\right)=x_{k}^{*-}$.

Induction step. to show: $c(g(x))<c(x) \Longrightarrow c(g(x+\Delta x))<c(x+\Delta x)$

First, consider $\Delta x$ such that segment borders are not crossed and define $x_{i+1}=$ $x_{i}+\Delta x$.

$$
\begin{aligned}
& c(g(x+\Delta x))<c(x+\Delta x) \\
\Longleftarrow & (A-s(x) c(g(x)))^{2}+2 \varepsilon \Delta x x^{g}<(A-s(x) c(x))^{2} .
\end{aligned}
$$

Since $A \leq c(g(x))<c(x)$ was assumed the above inequality holds, given that $\Delta x>0$ and $x^{g}<0$. So far only $\Delta x$ were considered, such that $x$ and $x+\Delta x$ are situated within the same segment. However analog to (ii.1) the argument also holds for $\Delta x$ that induce a shift across segment borders: In that case $\Delta x$ can be rewritten as $\Delta x=\sum_{i=1}^{n} \Delta x_{i}$ and the above argument can be applied sequentially passing from segment border to segment border: We choose $\Delta x_{i}$, such that for each $l$ the $x$-value $x+\sum_{i=1}^{l} \Delta x_{i}$ corresponds to a segment border.

$c\left(g\left(x+\sum_{i=1}^{l} \Delta x_{i}\right)\right)<c\left(x+\sum_{i=1}^{l} \Delta x_{i}\right) \Longrightarrow c\left(g\left(x+\sum_{i=1}^{l+1} \Delta x_{i}\right)\right)<c\left(x+\sum_{i=1}^{l+1} \Delta x_{i}\right)$.

Induction start to show: $c\left(x^{g}\right)=c(g(0))<c(0)$

This follows from the observation that $c(x)$ increases monotonically with $x$ for $c>A$ and $x<0$. 
Conclusion. While starting at $x=0$ and increasing $\Delta x$, the induction above showed that $c(g(x))<c(x)$ has to hold. Hence, before $x_{k}^{\mathrm{c}+}$ with $c\left(x_{k}^{\mathrm{c}+}\right)=A$ is reached, the point $c(g(x))=A=c\left(x_{k}^{*-}\right)$, when the trajectory reaches $c=A$ again, has to be passed. Therefore, $x_{k}^{*-}>x_{k}^{\mathrm{c}-}=g\left(x_{l}^{\mathrm{c}+}\right.$. The trajectory reaches $c=A$ to the right of the left border of $F_{k}^{\text {in- }}$.

Short recapitulation. In (ii) it was shown that a trajectory leaving the funnel exit point of the $k$ th funnel on the right $\left(F_{k}^{\mathrm{o}+}\right)$ reaches $c=A$ again on the left within the funnel entrance region of funnel $k, F_{k}^{\text {in- }}$.

We set out to show that the trajectory passing through the funnel exit point $F_{k}^{\text {o+ }}$ is a cycle. We claimed that the trajectory passes sequentially through the following states (equation 3.6):

$$
F_{k}^{\mathrm{o}+} \stackrel{(i i)}{\longrightarrow} F_{k}^{\mathrm{in}-} \stackrel{(i)}{\longrightarrow} F_{k}^{\mathrm{o}-} \stackrel{(*)}{\longrightarrow} F_{k}^{\mathrm{in}+} \stackrel{(i)}{\longrightarrow} F_{k}^{\mathrm{o}+}
$$

While validity of the state transitions $(i)$ directly follows from the assumption that both funnels $F_{k}^{ \pm}$are closed we showed in (ii) that the transition (ii) also holds. The argument showing that the transition $(*)$ occurs is fully analog to the argument in (ii) and left to the appendix (section C). Therefore we have shown that indeed the trajectory passing through $F_{k}^{\text {o+ }}$ is a cycle. In an earlier section we characterized a structure called funnel and showed that as long as a funnel is closed all trajectories passing the funnel entrance leave the funnel as a single trajectory passing through the funnel exit point $F_{k}^{\circ}$. Taken together this shows that as long as the parameters are chosen such that both funnels $k$ are closed there exists a region in phase space where trajectories converge in finite time onto limit cycles.

\subsubsection{Proof for Finite Time Convergence to Limit Cycles - Detailed Proof}

In this proof we want to show the following:

Parameters can be chosen such that $\varepsilon<\varepsilon_{k, c}, \varepsilon_{k, c}(A, \varphi)$ is a critical value, that depends on the system parameter $A$ and $\varphi$ and on the index $k$. If the inequality holds, then there exists a region in phase space $\left(\exists F_{k}^{i n} \subset \mathbb{R}^{2}, F_{k}^{i n} \neq\{\}\right)$, such that all trajectories passing through this space (all trajectories with $(x(t), c(t)) \in F_{k}^{\text {in }}$ ) will converge in finite time onto a single trajectory. This trajectory is a cycle.

Section (A) demonstrates the existence of finite time convergence: Trajectories passing through a non-empty set of points $F_{k}^{\text {in }}$ all reach the same point after a finite time $t$ if $\varepsilon<\varepsilon_{k, c}$.

Section (B) demonstrates the existence of cycles. Trajectories passing through the joined point of the previous section will revisit this point.

In Section $(C)$ we show that all trajectories within the region of phase space where (A) and (B) occur converge in finite time to limit cycles. 


\section{A - Finite Time Convergence within a Funnel Structure}

In this section we want to show the existence of finite time convergence of trajectories passing through a specific region onto a single trajectory, if $\varepsilon$ is smaller than a critical value $\left(\varepsilon<\varepsilon_{c}\right)$. To do so we define a structure called funnel $F$ (see figure 3.6), all funnels $F$ are situated within the region $-A<c<A$.

Funnel center $\boldsymbol{x}_{\boldsymbol{k}}^{\mathrm{c} \pm}$ and funnel identity $\boldsymbol{k}$. Each instance $F_{k}^{ \pm}$of this structure has a funnel center at position $x_{k}^{\mathrm{c} \pm}$ :

For $0<\varphi<\pi$ :

$$
\begin{aligned}
& x_{k}^{\mathrm{c}+}:=\varphi+\frac{3}{2} \pi+2(k-1) \pi \text { for } x>0, \\
& x_{k}^{\mathrm{c}-}:=\varphi-\frac{3}{2} \pi-(2 k-1) \pi \text { for } x<0,
\end{aligned}
$$

and for $-\pi<\varphi<0$ :

$$
\begin{array}{ll}
x_{k}^{\mathrm{c}+}:=\varphi+\frac{3}{2} \pi+2 k \pi & \text { for } x>0, \\
x_{k}^{\mathrm{c}-}:=\varphi-\frac{3}{2} \pi-(2 k-1) \pi & \text { for } x<0,
\end{array}
$$

$k \in 1, \ldots, K$ with $K \in \mathbb{N}$ is the funnel identity, funnels are counted outwards from $x=0$ on both sides, ' + ' and ' - ' indicate positive $(x>0)$ and negative $(x<0)$ side respectively, while c stands for funnel center.

Because $\frac{x_{k}^{\mathrm{c}}-\varphi-\frac{\pi}{2}}{\pi}=\left\lfloor\frac{x_{k}^{\mathrm{c}}-\varphi-\frac{\pi}{2}}{\pi}\right\rfloor$ and $s\left(x_{k}^{\mathrm{c} \pm}\right)=(-1)^{\left(\frac{x_{k}^{\mathrm{c}}-\varphi-\frac{\pi}{2}}{\pi}\right)}=-1$, all $x_{k}^{\mathrm{c}}$ lie at a border between $S^{\uparrow}$ on the left and $S^{\downarrow}$ on the right.

Funnel entrance region $\boldsymbol{F}_{\boldsymbol{k}}^{\text {in } \pm}$ : Further each funnel $F_{k}^{ \pm}$has a funnel entrance or input region:

$$
\begin{aligned}
F_{k}^{\mathrm{in}+} & :=\left\{(x, c=-A) \mid x \in\left[x_{k}^{\mathrm{c}+}-\pi, x_{k}^{\mathrm{c}+}+\pi\right)\right\} \\
F_{k}^{\mathrm{in}-} & :=\left\{(x, c=A) \mid x \in\left[x_{k}^{\mathrm{c}-}-\pi, x_{k}^{\mathrm{c}-}+\pi\right)\right\} .
\end{aligned}
$$

Because $x_{k}^{\mathrm{c}}$ lies at the border between $S^{\uparrow}$ and $S^{\downarrow}$ the left half of a funnel entrance belongs to $S^{\uparrow}$ and the right half to $S^{\downarrow}$. Note that all funnel entrances either lie fully within the negative or fully within the positive region: Because $x_{1}^{\mathrm{c}-}+\pi<0$ all $x \in F_{k}^{\mathrm{c}-}<0$ and because $x_{1}^{\mathrm{c}+}-\pi>0$ all $x \in F_{k}^{\mathrm{c}+}>0$.

Funnel exit point $\boldsymbol{F}_{\boldsymbol{k}}^{\mathbf{o}}$. Each funnel has an output or exit point:

$$
\begin{aligned}
& F_{k}^{\mathrm{o}+}:=\left(x=x_{k}^{\mathrm{c}+}, c=A\right) \\
& F_{k}^{\mathrm{o}-}:=\left(x=x_{k}^{\mathrm{c}-}, c=-A\right)
\end{aligned}
$$

The flow within a funnel. The incentive of this section, which is to show finite time convergence, can be restated in the following way: 
We want to show that all trajectories $(x(t), c(t))$ passing the funnel input region $F_{k}^{\text {in士 }}$ will eventually reach the funnel exit point $F_{k}^{o \pm}$ if $\varepsilon<\varepsilon_{k, c}$ :

$$
F_{k}^{\mathrm{in}+} \rightarrow F_{k}^{\mathrm{o}+} ; F_{k}^{\mathrm{in}-} \rightarrow F_{k}^{\mathrm{o}-}
$$

Here the arrow ' $F_{1} \rightarrow F_{2}$ ' is defined as

$$
\begin{aligned}
& \exists t_{1} \mid\left(x\left(t_{1}\right), c\left(t_{1}\right)\right) \in F_{1} \\
\wedge & \exists \Delta t>0 \mid t_{2}=t_{1}+\Delta t \wedge\left(x\left(t_{2}\right), c\left(t_{2}\right)\right) \in F_{2} .
\end{aligned}
$$

Flow in $\boldsymbol{x}$-direction. As shown above the left half of a funnel belongs to $S^{\uparrow}(s(x)=$ 1 ). From the system definition (equation 3.3 it follows that the flow in $x$-direction in an up shifted segment points to the right $(\dot{x}>0)$ while $c<A$. The right half of any funnel belongs to a down shifted segment $S^{\downarrow}(s(x)=-1)$. From the system definition (equation 3.3 it follows that the flow in $x$-direction in a down shifted segment points to the left $\dot{x}<0$ while $c>-A$. Therefore the flow in $x$-direction within a funnel region $\left(-A<c<A\right.$ and $x \in\left[x_{k}^{\mathrm{c} \pm}-\pi, x_{k}^{\mathrm{c} \pm}+\pi\right)$ points everywhere to the funnel center $x_{k}^{\mathrm{c} \pm}$. While $-A<c<A$ any trajectory that passed through the funnel entrance $F_{k}^{\mathrm{in} \pm}$ will move towards the respective funnel center $x_{k}^{\mathrm{c} \pm}$.

Flow in $c$-direction. From the system definition (equation 3.3 it follows that $\dot{c}<0$ if $x<0$ and vice versa. Therefore within a funnel region on the left $(-A<c<A$ and $x \in\left[x_{k}^{\mathrm{c}-}-\pi, x_{k}^{\mathrm{c}-}+\pi\right)$ the flow in $c$-direction points downwards everywhere $(\dot{c}<0$. Within a funnel region on the right $\left(-A<c<A\right.$ and $x \in\left[x_{k}^{\mathrm{c}+}-\pi, x_{k}^{\mathrm{c}+}+\pi\right)$ the flow in $c$-direction points upwards everywhere $(\dot{c}>0$. Trajectories passing through a left funnel entrance $F_{k}^{\text {in- }}$ will continue moving downwards until $c=-A$ : $\dot{c}<$ 0 . Trajectories passing through a right funnel entrance $F_{k}^{\text {in }+}$ will continue moving upwards until $c=A: \dot{c}>0$.

Bounding trajectories passing through the funnel entrance borders. Trajectories cannot cross each other. Further, as just demonstrated, all trajectories passing through a left funnel entrance $F_{k}^{\text {in- }}$ will move down in $c$-direction and towards the funnel center in $x$-direction. Hence, all these trajectories are bounded from below by the two trajectories passing through the funnel entrance borders (specifically through the points $\left(x_{l}, c_{l}=A\right) \mid x_{l}=\min \left(\left[x_{k}^{\mathrm{c}-}-\pi, x_{k}^{\mathrm{c}-}+\pi\right)\right)$ and $\left(x_{r}, c_{r}=\right.$ A) $\left.\mid x_{r}=\max \left(\left[x_{k}^{\mathrm{c}-}-\pi, x_{k}^{\mathrm{c}-}+\pi\right)\right)\right)$. All trajectories passing through a right funnel entrance $F_{k}^{\text {in }}$ will move up in $c$-direction and towards the funnel center in $x$-direction. Hence, all these trajectories are bounded from above by the two trajectories passing through the funnel entrance borders (specifically through the points $\left(x_{l}, c_{l}=-A\right) \mid x_{l}=$ $\min \left(\left[x_{k}^{\mathrm{c}+}-\pi, x_{k}^{\mathrm{c}+}+\pi\right)\right)$ and $\left.\left(x_{r}, c_{r}=A\right) \mid x_{r}=\max \left(\left[x_{k}^{\mathrm{c}+}-\pi, x_{k}^{\mathrm{c}+}+\pi\right)\right)\right)$.

Open and closed funnels. Because all trajectories passing through a funnel entrance are bounded by the two trajectories passing through the funnel entrance borders, if these two trajectories reach the respective funnel center $x_{k}^{\mathrm{c} \pm}$ before reaching the funnel exit point $F_{k}^{\mathrm{o}}$, this is true for all trajectories passing through the funnel entrance. 
Therefore, in that case all trajectories leave the funnel at the funnel exit point $F_{k}^{\circ \pm}$. In that case we call a funnel closed otherwise open (see figure 3.6).

Conditions for a funnel to be closed. The condition for a funnel to be closed follows from equation 3.5. For the trajectory passing through the outer left border, set $x_{\circ}=$ $x_{k}^{\mathrm{c} \pm}-\pi$ and $\Delta x=\pi$, for the trajectory passing through the right funnel border, set $x_{\circ}=x_{k}^{\mathrm{c} \pm}+\pi$ and $\Delta x=\pi$. For funnels on the left, set $c_{\circ}=A$, else $c_{\circ}=-A$.

For a left funnel:

$$
-A<s A \pm \sqrt{\left(A-s c_{\circ}\right)^{2}-\varepsilon \Delta x\left(2 x_{\circ}+\Delta x\right)}
$$

For a right funnel:

$$
A>s A \pm \sqrt{\left(A-s c_{\circ}\right)^{2}-\varepsilon \Delta x\left(2 x_{\circ}+\Delta x\right)}
$$

By rewriting these inequalities one obtains a critical parameter each for the trajectory passing through the inner and outer funnel border. For the trajectory passing through the outer funnel border the critical parameter is:

$$
\frac{4 A^{2}}{\pi\left(2\left|x_{k}^{\mathrm{c}}\right|+\pi\right)}=\varepsilon_{k, c}^{\mathrm{o}} .
$$

For the trajectory passing through the inner funnel border the critical parameter is:

$$
\frac{4 A^{2}}{\pi\left(2\left|x_{k}^{\mathrm{c} \pm}\right|-\pi\right)}=\varepsilon_{k, c}^{\mathrm{i} \pm}
$$

If the time constant $\varepsilon$ is small enough such that it is smaller than both critical parameters the funnel is closed $\left(\varepsilon<\varepsilon_{k, c}^{\mathrm{i} \pm}\right.$ and $\left.\varepsilon<\varepsilon_{k, c}^{\mathrm{o} \pm}\right)$. The outer condition is harder to be met, hence, a funnel is closed if $\varepsilon$ is smaller than the first critical parameter (equation $3.7 . \varepsilon<\varepsilon_{k, c}^{\mathrm{o}}$ ).

Defining $\varepsilon_{k, c}$. So far we have considered a critical parameter for each funnel. Depending on $\varphi$ either the critical parameter for funnel $k$ on the left or the critical parameter on the right side is larger $\left(\varepsilon_{k, c}^{\mathrm{o}+}>\varepsilon_{k, c}^{\mathrm{o}-}\right.$ or $\left.\varepsilon_{k, c}^{\mathrm{o}+}<\varepsilon_{k, c}^{\mathrm{o}-}\right)$. We will call the smaller of the two $\varepsilon_{k, c}$. If $\varepsilon<\varepsilon_{k, c}$, both funnels, on the left and on the right, are necessarily closed.

\section{B - Existence of Limit Cycles}

In the previous section we showed that any trajectory entering into a funnel entrance will leave that respective funnel at its funnel exit point if the funnel is closed. In the following section we assume that the parameters are chosen such that the considered funnels are closed.

Proving the existence of cycles only requires to show how funnels connect. Here we show that a trajectory passing through the funnel exit point $F_{k}^{\mathrm{o}+}$ will eventually reach this point again: $F_{k}^{\mathrm{o}+} \rightarrow F_{k}^{\mathrm{o}+}$, hence completing a full cycle. Specifically the 
trajectory starting at the funnel exit point of the $k$ th funnel on the right side $\left(F_{k}^{\mathrm{o}+}\right)$ passes through a sequence of states which are reaching the funnel entrance region of the $k$ th left funnel $\left(F_{k}^{\text {in- }}\right)$, then continuous to the respective funnel exit point $\left(F_{k}^{\mathrm{o}-}\right)$. This exit point in turn connects to the funnel entrance region of the $k$ th funnel on the right $\left(F_{k}^{\text {in }+}\right)$ and hence the trajectory will continue to the respective funnel exit point completing the full cycle. We want to show that

$$
F_{k}^{\mathrm{o}+} \stackrel{(i i)}{\longrightarrow} F_{k}^{\mathrm{in}-} \stackrel{(i)}{\longrightarrow} F_{k}^{\mathrm{o}-} \stackrel{(*)}{\longrightarrow} F_{k}^{\mathrm{in}+} \stackrel{(i)}{\longrightarrow} F_{k}^{\mathrm{o}+} .
$$

(This equation is identical to formula 3.6 in section 3.3.3.)

The steps $(i)$ were proven in the section A. Step (*) is fully analog to step $(i i)$ and included in the appendix (section $\mathrm{C}$ ). Hence only step (ii) remains to be proven (see figure 3.7).

$x_{k}^{*-}$ - the position where the trajectory returns to $c=A$. Here we study the trajectory $(x(t), c(t))$ leaving at the funnel exit point $F_{k}^{o+}$ and its corresponding curve $c(x)$. From the system definition (equation 3.3 it follows that while $c>A$ and $x>0$ the trajectory is monotonically increasing in $c(\dot{c}>0)$ and monotonically decreasing in $x(\dot{x}<0)$. Eventually the trajectory passes $x=0$. While $c>A$ and $x<0$ the trajectory is monotonically decreasing in $x$ and in $c(\dot{x}<0$ and $\dot{c}<0$ until the trajectory reaches $c=A$ ). We denote the $x$-position of this return as $x^{*-}$.

$$
x_{k}^{*-} \in F_{k}^{\mathrm{in}-} \Longleftrightarrow x_{k}^{\mathrm{c}-}-\pi \stackrel{(i i .2)}{\leq} x_{k}^{*-} \stackrel{(i i .1)}{<} x_{k}^{\mathrm{c}-}+\pi
$$

Therefore showing the transition $F_{k}^{\mathrm{o}+} \stackrel{(i i)}{\longrightarrow} F_{k}^{\text {in- }}$ is equivalent to showing that the two inequalities (ii.1) and (ii.2) hold.

Replacing the trajectory leaving at $\boldsymbol{F}_{k}^{\mathbf{o}+}$ by the function $\boldsymbol{c}(\boldsymbol{x})$. We are only interested in the part of the trajectory from leaving the funnel exit point $F_{k}^{\mathrm{o}+}$ until reaching $c=A$ again. This part of the trajectory can be expressed through the corresponding curve $c(x)$ In the paragraph 3.3 .2 (remarks on monotonicity) it was discussed that analog to the behavior of the trajectory any connected curve $c(x)$ while $c>A$ is strictly monotonically decreasing with $x$ while $x>0$ and strictly monotonically increasing while $x<0$. Because of the monotonicity the curve $c(x)$ with $c \geq A$ is a function, there is exactly one $c$-value assigned to any $x$-value. The following part of the proof concerns the curve $c(x)$ within this region.

(ii.1) To Show: $x_{k}^{*-}<x_{k}^{\mathrm{c}-}+\pi$; $x_{k}^{*-}$ Lies Left of the Right Entrance of $F_{k}^{\text {in- }}$

Defining $\boldsymbol{x}^{\mathbf{f}}$ and $\boldsymbol{f}(\boldsymbol{x})$. The function $f(x)$ is defined in the following way (see figure 3.8):

$$
f(x):=-x+x^{\mathrm{f}}
$$

$x^{\mathrm{f}}$ is defined such that it has the same distance to the funnel exit point $F_{k}^{\mathrm{o}+}$ as the right border of the funnel entrance $F_{k}^{\mathrm{in}-}$ from $x=0$ :

$$
0-\left(x_{k}^{\mathrm{c}-}+\pi\right)=x_{k}^{\mathrm{c}+}-x^{\mathrm{f}} .
$$


Now we show that with the above definition it follows that $x^{\mathrm{f}}>0$ :

For $0<\varphi<\pi$ :

$$
x^{\mathrm{f}}=\varphi+\frac{3}{2} \pi+2(k-1) \pi+\varphi-\frac{3}{2} \pi-(2 k-1) \pi+\pi=2 \varphi>0 .
$$

For $-\pi<\varphi<0$ :

$$
x^{\mathrm{f}}=\varphi+\frac{3}{2} \pi+2 k \pi+\varphi-\frac{3}{2} \pi-(2 k-1) \pi+\pi=2 \varphi+2 \pi>0 .
$$

In the following we show that $x$ and $f(x)$ are situated within the same segment type. Both either lie within an up shifted or a down shifted segment: $s(x)=s(f(x))$.

For $0<\varphi<\pi$ as well as $-\pi<\varphi<0$ :

$$
\begin{aligned}
& (-1)^{\left\lfloor\frac{f(x)-\varphi-\frac{\pi}{2}}{\pi}\right\rfloor}=(-1)^{\left\lfloor\frac{-x+x^{\mathrm{f}}-\varphi-\frac{\pi}{2}}{\pi}\right\rfloor} \\
= & (-1)^{\left\lfloor\frac{-\left(x-\varphi-\frac{\pi}{2}\right)}{\pi}+1\right\rfloor}=(-1)^{\left\lfloor\frac{x-\varphi-\frac{\pi}{2}}{\pi}\right\rfloor}
\end{aligned}
$$

The equality holds for geometrical reasons.

Induction. to show: $c\left(x_{k}^{c-}+\pi\right)=c\left(f\left(x_{k}^{c+}\right)\right)>c\left(x_{k}^{c+}\right)=A$.

Induction step. to show: $c(f(x))>c(x) \Longrightarrow c(f(x+\Delta x))>c(x+\Delta x)$ with $\Delta x>0$.

$\Delta x$ is chosen such that segment borders are not crossed and we define $x_{i+1}=$ $x_{i}+\Delta x$. The right side of the equation is considered:

$$
\begin{aligned}
c\left(f\left(x_{i+1}\right)\right)=c\left(-x_{i+1}+x^{f}\right)=c\left(-x_{i}-\Delta x+x^{f}\right)=c\left(f\left(x_{i}\right)+(-\Delta x)\right) & \\
& >c\left(x_{i+1}\right)=c\left(x_{i}+\Delta x\right) \\
\Longleftrightarrow & s\left(f\left(x_{i}\right)\right) A+\sqrt{\left(A-s\left(f\left(x_{i}\right)\right) c\left(f\left(x_{i}\right)\right)\right)^{2}-\varepsilon(-\Delta x)\left(2 f\left(x_{i}\right)+(-\Delta x)\right)} \\
& >s\left(x_{i}\right) A+\sqrt{\left(A-s\left(x_{i}\right) c\left(x_{i}\right)\right)^{2}-\varepsilon(\Delta x)\left(2 x_{i}+\Delta x\right)} \\
\Longleftarrow & \left(A-s\left(x_{i}\right) c\left(f\left(x_{i}\right)\right)\right)^{2}-\varepsilon \Delta x\left(-2\left(-x_{i}+x^{f}\right)+\Delta x\right) \\
& >\left(A-s\left(x_{i}\right) c\left(x_{i}\right)\right)^{2}-\varepsilon \Delta x\left(2 x_{i}+\Delta x\right) \\
\Longleftrightarrow & \left(A-s\left(x_{i}\right) c\left(f\left(x_{i}\right)\right)\right)^{2}+2 \varepsilon \Delta x x^{f}>\left(A-s\left(x_{i}\right) c\left(x_{i}\right)\right)^{2}
\end{aligned}
$$

The inequality holds because we assumed $c\left(f\left(x_{i}\right)\right)>c\left(x_{i}\right)$ and $c \geq A>0$ and $s(x)=$ $s(f(x))$ holds. So far we assumed staying within the same segment, however, because $s(x)=s(f(x))$, incrementally adding $\Delta x$ going from segment border to segment border keeps the above reasoning intact, $c(f(x))>c(x)$ holds true.

Induction start. to show: $c\left(f\left(x^{f}\right)\right)=c(0)>c\left(x^{f}\right)$

This directly follows from section 3.3.2(Remarks on monotonicity). 
Conclusion. It follows that when passing the right funnel entrance border of funnel $F_{k}^{-}$the trajectory does not enter the funnel yet: $c\left(x_{k}^{\mathrm{c}-}+\pi\right)=c\left(f\left(x_{k}^{\mathrm{c}+}\right)\right)>c\left(x_{k}^{\mathrm{c}+}\right)=A$.

Section 3.3.2 (Remarks on monotonicity) stated that $c(x)$ is monotonically increasing with $x$ for $x<0$ and $c>A$. It follows that the trajectory will reach $c=A$ to the left of the right funnel entrance border of $F_{k}^{-}: x_{k}^{*-}<x_{k}^{\mathrm{c}-}+\pi$.

\section{(ii.2) To Show: $\mathrm{x}_{\mathrm{k}}^{\mathrm{c}-}+\pi \leq \mathrm{x}^{*} ; x_{k}^{*-}$ Lies Right of the Left Entrance of $F_{k}^{\mathrm{in}-}$}

To show that the trajectory reaches the funnel entrance to the right of the left funnel border of funnel $k$, we employ a strategy that is analog to(ii.1) in most parts.

Defining $\boldsymbol{x}^{\mathbf{g}}$ and $\boldsymbol{g}(\boldsymbol{x})$. The function $g(x)$ is defined in the following way (see figure 3.9 :

$$
g(x)=-x+x^{\mathrm{g}}
$$

$x^{\mathrm{g}}$ is defined such that it has the same distance to the left border of the funnel entrance $F_{k}^{\text {in- }}$ as the funnel exit point $F_{k}^{\mathrm{o}+}$ from $x=0$.

$$
x_{\mathrm{g}}-\left(x_{k}^{\mathrm{c}-}-\pi\right)=x_{k}^{\mathrm{c}+}-0 .
$$

From the above definition of $x^{\mathrm{g}}$ it follows that $x^{\mathrm{g}}<0$ :

For $0<\varphi<\pi$ :

$$
x^{g}=\varphi+\frac{3}{2} \pi+2(k-1) \pi+\varphi-\frac{3}{2} \pi-(2 k-1) \pi-\pi=2 \varphi-2 \pi>0 .
$$

For $-\pi<\varphi<0$ :

$$
x^{g}=\varphi+\frac{3}{2} \pi+2 k \pi+\varphi-\frac{3}{2} \pi-(2 k-1) \pi-\pi=2 \varphi>0 .
$$

Now we will show that $x$ and $g(x)$ are situated within the same segment type: $s(x)=$ $s(g(x))$. For $0<\varphi<\pi$ as well as $-\pi<\varphi<0$ :

$$
\begin{aligned}
& (-1)^{\left\lfloor\frac{g(x)-\varphi-\frac{\pi}{2}}{\pi}\right\rfloor}=(-1)^{\left\lfloor\frac{-x+x^{g}-\varphi-\frac{\pi}{2}}{\pi}\right\rfloor} \\
= & (-1)^{\left\lfloor\frac{-\left(x-\varphi-\frac{\pi}{2}\right)}{\pi}+1\right\rfloor}=(-1)^{\left\lfloor\frac{x-\varphi-\frac{\pi}{2}}{\pi}\right\rfloor}
\end{aligned}
$$

The equality holds for geometrical reasons.

Induction. to show: When increasing $x, c\left(g\left(x^{\prime}\right)\right)=c\left(x_{k}^{*-}\right)=A$ occurs before $c\left(x_{k}^{c+}\right)=$ $A$ and hence $g\left(x_{k}^{c+}\right)=x_{k}^{c-}-\pi<g\left(x^{\prime}\right)=x_{k}^{*-}$.

Induction step. to show: $c(g(x))<c(x) \Longrightarrow c(g(x+\Delta x))<c(x+\Delta x)$

$\Delta x$ is chosen such that segment borders are not crossed and we define $x_{i+1}=$ 
$x_{i}+\Delta x$. The right hand side is considered:

$$
\begin{aligned}
& c\left(g\left(x_{i+1}\right)\right)<c\left(x_{i+1}\right)=c\left(x_{i}+\Delta x\right) \\
\Longleftarrow & \left(A-s(x) c\left(g\left(x_{i}\right)\right)\right)^{2}+2 \varepsilon \Delta x x^{g}<\left(A-s(x) c\left(x_{i}\right)\right)^{2} .
\end{aligned}
$$

Assuming $A \leq c(g(x))<c(x)$ the above inequality follows, given that $\Delta x>0$ and $x^{\mathrm{g}}<0$.

So far it was assumed that $\Delta x$ is chosen such that $x+\Delta x$ remains within the same segment as $x$, however, because $s(x)=s(g(x))$, incrementally adding $\Delta x$ going from segment border to segment border keeps the above reasoning intact, $c(g(x))<c(x)$ holds true.

Induction start. to show: $c\left(x^{\mathrm{g}}\right)=c(g(0))<c(0)$

This directly follows from 3.3 .2 (Remarks on monotonicity).

Conclusion. While starting at $x=0$ and increasing $\Delta x$, the induction above showed that $c(g(x))<c(x)$ has to hold. Hence, before $x_{k}^{\mathrm{c}+}$ with $c\left(x_{k}^{\mathrm{c}+}\right)=A$ is reached, the point $c(g(x))=A=c\left(x^{*-}\right)$, when the trajectory reaches $c=A$ again, has to be passed. Therefore, $x^{*-}>x_{k}^{\mathrm{c}-}-\pi=g\left(x_{k}^{\mathrm{c}+}\right)$. The trajectory reaches $c=A$ to the right of the left border of $F_{k}^{\mathrm{in}-}$.

Short recapitulation. Overall in (ii) we have shown that the trajectory leaving the funnel exit point of the funnel $F_{k}^{+}$reaches between the left and right funnel entrance borders of the funnel $F_{k}^{-}$. In (i) we showed that if $\varepsilon_{c}>\varepsilon$ this trajectory will leave this funnel at the funnel exit point $F_{k}^{(o)-}$.

To complete the cycle it remains to show that a trajectory leaving at the funnel exit point of the $k$ th funnel on the left $F_{k}^{o-}$ will enter the funnel entrance of the $k$ th funnel on the right $F_{k}^{\mathrm{in}+}$. The argument is fully analog to the one presented above in (ii) and is presented in the appendix (section C).

\section{C - Funnels are Dense}

This section demonstrates that within the region of phase space with closed funnels all trajectories converge in finite time to limit cycles. Specifically we want to show that the funnel structure is dense. There are no gaps between funnel entrances:

$$
\left\{x \mid x \in\left[x_{m}^{\mathrm{c}+}-\pi, x_{n}^{\mathrm{c}+}+\pi\right) \wedge x \notin F_{m}^{\mathrm{in}+}, \ldots, F_{n}^{\mathrm{in}+}\right\}=\{\}
$$

This follows directly from the definition of $F_{k}^{\text {in } \pm}$.

\subsection{Discussion}

In this chapter we constructed a minimal model of adaptation, considering an adapting entity in a slowly changing environment. The system was realized as an interactive system between a potential and a particle moving inside it. The potential, a superposition of a sinusoidal function and a straight line, slowly changes by adjusting the 
straight line's slope depending on the particle's position. Since the particle follows the local gradient to minimize the energy, an overall seesaw behavior emerges. The phase space is characterized by seemingly infinite nested limit cycles. A proof for the existence of limit cycles was provided for a simplified system sharing the qualitative phase space portrait of nested limit cycles. Even this minimal model of an adaptive system, described by only two smooth ODEs, shows rich dynamics. Due to the system's simplicity other research fields converge onto similar equations. In the following discussion we consider the system from those different perspectives.

\section{The System as a Second Order Differential Equation.}

The dynamics of this system are fully described by two ODEs. Indeed, they can be rewritten as a single second order ODE:

$$
\frac{1}{\varepsilon} \ddot{x}=-A \cos \left(\frac{1}{\varepsilon} \dot{x}-\varphi\right)-x
$$

Interpretation as a mechanical oscillator. This reformulation allows for an interpretation as a mechanical oscillator such as a mass hanging from a spring.

In this framework $\frac{1}{\varepsilon}$ can be understood as the mass. $x$ is the extension of the spring. The last term $-x$ is the restoring force of the spring with a spring constant of 1 . The first term on the right resembles a damping force, because it depends on the velocity $\dot{x}$. However, opposed to the damping force in a passive spring oscillator which is proportional to the current velocity of the mass, the dependence of the acceleration on the velocity is mediated by a cosine function. For some velocities the particle is accelerated while for others it is slowed down.

There is an alternative way to rewrite the system as a second order ODE which is presented in the appendix in section B.

Without the unusual damping term the equation above describes a harmonic oscillator $\left(\frac{1}{\varepsilon} \ddot{x}=-k x\right.$ with $k \geq 0$, here $\left.k=1\right)$. Through decreasing the parameter $A$ the system transitions into a harmonic oscillator. It remains open to study how this transition looks like. Harmonic oscillators are broadly studied in physics, since they are very simple and many oscillatory systems can be reduced to a harmonic oscillator by leading order approximation. A negative damping term is often added to such an oscillator to account for energy loss of the system due to friction. An oscillator with friction will eventually reach a stable resting position. However, here the damping term sometimes has a positive sign, depending on the current velocity. Therefore, for some velocities energy is added to the system, a property not found in passive matter.

Active matter. In recent years biophysics has focused more and more on studying self-propelled particles or active matter. These particles consume energy from their environment to convert it into motion. Examples of self-propelled particles include animals such as birds moving in flocks and fish moving in schools. Furthermore, there are self-propelled bacteria and other microorganisms. Also artificial systems, such as (nano-)robots moving in a swarm exist. Many such self-propelled particles interacting make up so called active matter. They may produce intricate collective dynamics emerging from very simple behavior rules guiding each participating particle. 
The dependence between acceleration and velocity. In the system presented here acceleration depends on velocity via a sinusoidal function; across the velocity dimension regions with acceleration and deceleration alternate. There are examples in the literature of particles, which show an acceleration-velocity-dependence with a simpler structure: One idea is based on empirical evidence that the motion dynamics of some chemotactic bacteria, such as granulocytes, can be separated into two independent processes for direction and speed control. The later can be described by a steering mechanism, which leads to acceleration for slow speeds and deceleration for large speeds [27, 73]. Another idea relates to the finding that some marine bacteria temporarily store the energy gained from the environment through nutrition in an internal energy depot. The internal energy dynamics depend on the conversion of stored energy into motion as well as internal dissipation of energy used for metabolic processes. Assuming fast internal energy dynamics compared to motion control leads to a situation with bacterial acceleration for slow speeds and deceleration due to friction for large speeds within a certain parameter regime [15, 74]. For a theoretical overview, see reference [71].

The oscillation amplitude as a property of the initial conditions and the system. The system presented here is an example of an active oscillator. Another active oscillator is the van der Pol oscillator:

$$
\ddot{x}=-\mu\left(x^{2}-1\right) \dot{x}-x
$$

with the parameter $\mu \geq 0$. The damping term $-\mu\left(x^{2}-1\right) \dot{x}$ is negative for large $|x|$ and positive for small $|x|$. In the phase plane spanned by $x$ and $\dot{x}$ the whole flow approaches a single limit cycle. The information regarding the initial conditions gets lost over time. The average amplitude of the oscillator is a property of the system.

In contrast the above mentioned harmonic oscillator is neutrally stable, the phase plane consists of infinitely many closed orbits. A slight perturbation of the system will change the long term behavior by shifting towards a neighboring orbit. The information regarding the initial conditions is conserved, the average amplitude of the oscillation depends only on the initial conditions.

The oscillator presented in this chapter shares properties of both system types. While the initial condition determines which limit cycle is approached, each limit cycle has a basin of attraction, such that many initial conditions eventually lead to the same long term behavior. The resulting average oscillation amplitude depends on the initial condition as well as on the system itself.

A computational perspective. Some dynamical systems may be very efficient in performing a tasks, potentially allowing for building new efficient computational tools 62 64. From a computational perspective the system studied in this chapter performs the operation of discretizing the analog signal provided by the initial conditions into discrete average oscillation amplitudes, a digital signal. Whether this operation could be exploited to perform interesting computational tasks should be explored in the future. However, the oscillator presented here is not the simplest system to achieve 
such a digitalization of oscillator amplitudes. Consider the following system:

$$
\begin{aligned}
& \dot{\phi}=\omega \\
& \dot{r}=\sin (r) .
\end{aligned}
$$

the angle $\phi$ rotates with a constant speed $\omega$. The amplitude $r$ gets discretized, since the sine function has infinitely many zero crossings. It remains an open question whether a computational application can be found in which the adaptive oscillator presented in this chapter is superior to the simpler oscillator presented above.

\section{Conceptualizing the System as Two Coupled Phase Oscillators}

The Adler equation was developed to understand the synchronization of a limit cycle oscillator with a weakly coupled periodic input of similar frequency or the synchronization between two weakly coupled limit cycle oscillators with similar intrinsic frequencies. The weak coupling assumption allows for approximating the evolution of each oscillator by a phase representation, the input is weighted by an infinitesimal phase response curve, which accounts for the varying susceptibility to inputs within the cycle. Further, it is assumed that both oscillators have similar frequencies, so that the phase difference between the two oscillators changes very slowly compared to the change in absolute phase. This slow change in relative phase allows for approximating the coupling function between the oscillators depending on the absolute phases of both oscillators by a function that only depends on the phase differences. This is achieved by averaging out effects of absolute phase across the completion of a cycle for any fixed phase difference. Subtracting the equations for the phase evolution of each oscillator from one another yields a differential equation, that defines the evolution of the phase difference between the oscillators. For sinusoidal coupling this equation is the Adler equation:

$$
\frac{\mathrm{d} \Delta \phi}{\mathrm{d} t}=\Delta \omega+\epsilon \sin (\Delta \phi)
$$

$\Delta \phi$ is the phase difference between the oscillators, $\Delta \omega=\omega_{2}-\omega_{1}$ is the difference of the intrinsic frequencies, $\epsilon$ is the coupling strength. The region in parameter space spanned by the coupling strength $\epsilon$ and the frequency difference $\Delta \omega$, for which this equation yields fixed points, is called Arnold tongue and indicates synchronization behavior [2, 35, 67].

The equation coincides with the first of the two ODEs describing the original oscillator studied in this chapter (see equation 3.2 . Therefore it may be possible to reinterpret this system as an interaction of two oscillators in the spirit of the Adler equation. Accordingly, the variable $x$ corresponds to $\Delta \phi \pm \varphi$, the phase difference between the two oscillators plus or minus a constant phase shift, and the variable $c$ corresponds to $\Delta \omega$, the difference in intrinsic frequency between the two oscillators. With this substitution equation 3.2 can be rewritten as such:

$$
\begin{aligned}
\frac{\mathrm{d} \Delta \phi}{\mathrm{d} t} & =-\Delta \omega-A \cos (\Delta \phi-\varphi) \\
\frac{\mathrm{d} \Delta \omega}{\mathrm{d} t} & =\varepsilon \Delta \phi .
\end{aligned}
$$


This system can be understood as the interplay of two opposing mechanisms: As long as the system operates within the Arnold tongue regime of the first equation the first mechanism dominates, which strives towards a phase locking between the two oscillators.

However, a second mechanism strives to restore the initial distance between the two oscillators, by dynamically adjusting the frequency difference $\Delta \omega=\omega_{2}-\omega_{1}$ in favor of the oscillator lacking behind. Note that $\Delta \phi$ was substituted for $x$ and is not a periodic variable. It measures the absolute distance between the two oscillators relative to a fixed starting position not the phase difference.

The dynamics discussed in this chapter arise from a competition: The first mechanism aims towards a relative phase difference $\Delta \phi$ measured in a periodic distance and the second aims at restoring distance in $\Delta \phi$ measured in a non-periodic absolute distance. The two oscillators stay in a phase locking Arnold tongue regime dominated by the first mechanism, until the influence of the second mechanism becomes strong enough to break free and the oscillators overtake each other due to differences in intrinsic frequencies. Eventually, the relative contribution of the first mechanism again becomes strong enough to dominate and so forth (for a different system dynamically moving in and out of the Arnold tongue regime, see reference [50]).

\subsection{Summary}

In this chapter we aimed at furthering the understanding of adaptation from a dynamical systems' perspective. We defined a minimal adaptive system. Numerical simulations revealed an intricate phase space topology of apparently infinitely many equally spaced nested limit cycles. This finding is robust across a wide range of parameters. Only if the phase shift is a multiple of $\pi$ numerical studies are inconclusive.

While the nonlinearity of the system prevented analytic treatment such analysis was possible for a simplified system. Numerical studies confirmed that the qualitative behavior of nested limit cycles in phase space was unharmed by the simplification. However, analytic treatment was possible. For a certain parameter range a funnel structure could be identified which is characterized by splitting the full flow, so that all trajectories entering into a specific closed funnel merge into a single one. This trajectory eventually reaches the funnel entrance of the corresponding funnel on the opposite side. The same logic applies here, all trajectories entering this funnel merge into one, which returns to the original funnel. This way the full cycle is closed and the existence of the numerically observed limit cycles is analytically proven. $\mathrm{Nu}-$ merical simulations indicate that the limit cycle structure exists beyond the region characterized by closed funnels.

In the discussion section alternative views on the system were proposed. These include understanding the system as an active oscillator or as a computational unit that discretizes analog input into oscillations with predefined average amplitudes. In the end we discussed the relation of the system to the Adler equation and reinterpreted the system as an interaction between two phase oscillators.

Context of the thesis. In this thesis we want to explore the fundamental dynamical properties of features often used in theoretical neuroscience. We ask whether emer- 
gent dynamical phenomena observed in neural networks can already be produced by extremely simple systems based on dynamical features typical to neural models. Neural networks often are made up of adaptive neural units. Even though the adaptive system studied in this chapter is very simple and is fully described by two ODEs, it already exhibits an intricate phase space topology of nested limit cycles. The system shows multistable oscillations. Multistability is considered to be an interesting feature of neural networks (e.g. see reference [55]). Here we show that even a two dimensional adaptive system can produce multistability with apparently infinitely many stable states. 



\section{Chapter 4}

\section{Reordering and Order Conservation in Symmetric All-to-All Pulse-Coupled Phase Oscillator Networks Depending on Self-Loop Strength}

\subsection{Introduction}

The goal of this thesis is to understand the implications of dynamical features often used to model biological, in particular neurological phenomena from a dynamical systems perspective. Units of neural networks commonly interact by exchanging pulses. In the next two chapters we study the effect of pulse-coupling in extremely simple networks. A special focus lies on coupling by exchanging delayed $\delta$-pulses. To understand the implications of pulse-coupling from a dynamical systems perspective we consider ODE systems as reference systems. In this chapter we choose to study an all-to-all pulse-coupled network of phase oscillators, that has full permutation symmetry.

Pulse-coupling. A pulse has a temporally extended characteristic shape of intensity. In a system with pulse-coupling from entity $a$ to entity $b$ a pulse is sent each time entity $a$ is in a specific state, e.g. reaches a threshold. The pulse is received by entity $b$ and interacts with it over the full time of pulse reception with varying intensity according to the shape of the pulse. Many examples of pulse-coupling come to mind from artificial systems, especially from electronics. But nature also provides us with examples: Interactions between tectonic plates can be modeled via pulse-coupling [32] as well as the synchronization behavior of the firefly species Photonius Carolinus via light pulses [57]. Another example is the circadian clock in mammals, that controls physiological processes following a day-night cycle. The clock is made up of a cascade of molecular reactions that needs approximately 24 hours to repeat itself. The control of processes in the body is achieved by secreting the hormone melatonin into the blood stream each night [4. For a precise definition of pulse-coupling see chapter 2.

Pulse-coupling between neurons. Communication between neurons in the brain is achieved by exchanging pulses. Input from other neurons is accumulated across the neural input sides as changes in membrane potential, the electrical potential between inside and outside of the cell. If the total depolarization at the soma, the cell body, crosses a voltage threshold an action potential is generated. An action potential is a large fluctuation in membrane potential, which has a stereotypical shape. This action 
potential then travels along the axon, a cable-like structure, to the output sides of the neuron, where it is connected to other neurons via electrical or chemical synapses. In the central nervous system, including the brain, synapses are predominantly chemical. They consist of a presynaptic part belonging to the sending neuron, the synaptic cleft, which is a small gap between the two neurons, and a postsynaptic part, belonging to the receiving neuron. If an action potential reaches such a synapse, it causes the ejection of small molecules, the neurotransmitters, from the presynaptic part into the synaptic cleft. These neurotransmitters travel to the postsynaptic part, and by binding at specific binding sites cause a change in membrane potential of the receiving neuron. The process from action potential generation at the soma and transmission and synaptic interaction once started occurs, as a first approximation, always in the same way. The combined effect is often modeled as a pulse triggered each time the membrane potential of the neuron reaches a threshold. In a minimal model the whole pulse is summarized into a single $\delta$-pulse that occurs after a fixed time delay after threshold crossing. Neural models with $\delta$-pulse-coupling can show rich dynamics [5, 17, 64]

Phase oscillators. Phase oscillators are very simple, while at the same time not settling into a steady state. Networks of phase oscillators are broadly studied in physics. One reason is that weakly coupled oscillators can be described as phase oscillators [35. Phase oscillator networks can give rise to interesting dynamical phenomena such as synchronization behavior [30, 57, clustering [8, 30] heteroclinic switching [8, 30], chaos [11, repeated firing patterns [17] or chimera states [1, 41]. Dynamics of phase oscillator networks including pulse-coupling have been discussed as well e.g. synchronization behavior [57, repeated firing patterns [17] or clustering and heteroclinic switching [62, 85]. While many phenomena can occur in pulse-coupled oscillator networks as well as networks fully described by ODEs, here we consider a case, where the two system types show qualitatively different behavior. For a definition of phase oscillators see chapter 2 .

Symmetry in networks. A fully symmetric network topology is a simple structure and yields symmetries in the dynamics. A dynamical system has a symmetry if there exists a transformation that keeps the set of trajectories in phase space unchanged. Systems of oscillators with full permutation symmetry often produce interesting phenomena e.g. cluster states [8, 11, 24, 30, 62, 81, 85], which may yield useful computational properties. 62 64.

Let

$$
\dot{x_{i}}=f_{i}\left(x_{1}, x_{2}, \ldots, x_{N}\right)
$$

describe the evolution of node $i$ in a network with $N$ nodes. Let $\sigma$ be a permutation of the numbers $1, \ldots, N$. Then $\sigma$ is a network symmetry, if

$$
\mathbf{f}(\sigma \mathbf{x}, t)=\sigma \mathbf{f}(\mathbf{x}, t) .
$$

In other words there is a network symmetry if it does not make a difference, whether the indices of the nodes are permuted or the functions describing the temporal evolution of each node. 
An all-to-all coupled network (without self-loops) of identical nodes and equal coupling has the full $S_{N}$ symmetry. All possible permutations of N numbers are symmetries of this network. In such a network the dynamic node equations take the following form (see reference [38]):

$$
\begin{aligned}
& \dot{x}_{1}=f\left(x_{1} ; \overline{x_{2}, x_{3}, \ldots, x_{N}}\right) \\
& \dot{x}_{2}=f\left(x_{2} ; \overline{x_{1}, x_{3}, \ldots, x_{N}}\right) \\
& \vdots \\
& \dot{x}_{N}=f\left(x_{N} ; \overline{x_{1}, x_{2}, \ldots, x_{N-1}}\right) .
\end{aligned}
$$

The overline indicates permutation symmetry of the variables (e.g. $f\left(x_{1} ; \overline{x_{2}, x_{3}, \ldots, x_{N}}\right)$ $=f\left(x_{1} ; \overline{x_{\sigma_{2}}, x_{\sigma_{3}} \ldots, x_{\sigma_{N}}}\right)$.

Golubitsky et al. 25] observed the following: If a network described by smooth ODEs has a symmetry with regards to node $i$ and node $j$, then the polydiagonal $x_{i}=x_{j}$ is flow invariant, i.e. any trajectory starting on this polydiagonal is confined to it. The polydiagonal separates the whole phase space into two volumes, each corresponding to a specific ordering of nodes $i$ and $j$. Because in a smooth ODE system the existence and uniqueness theorem holds, trajectories are continuous and cannot cross each other. It follows that they cannot cross the polydiagonal. This implies that node $i$ and node $j$ cannot overtake each other, their ordering is conserved, if they are oscillators, their average frequency will be the same and their winding number can maximally differ by 1 . In a system with full permutation symmetry $\left(\sigma \epsilon S_{N}\right)$ it follows that no node can overtake another, so order conservation is true for the whole network.

Even though it might be tempting to apply the same reasoning to pulse-coupled systems as well, Kielblock et al. [38] have demonstrated that overtaking can indeed take place in symmetrical systems of all-to-all pulse-coupled oscillators (see figure 4.2. panel A and B). They chose a coupling kernel that has no delay and does not induce any non-smoothness into the phase evolution of the receiving oscillator. Hence the breaking of order conservation should indeed be attributed to pulse-coupling. Kielblock et al. argue that this is possible despite the reasoning of Golubitsky et al. because the state space of pulse-coupled systems has more dimensions due to an additional dependence on pulse sending times, compared to ODE systems which show state dependent coupling.

Furthermore, Kielblock et al. provide an analysis for a network of all-to-all $\delta$-pulsecoupled oscillators and determine the parameter regime for which order conservation is guaranteed.

Self-coupling. Introducing self-coupling into the system studied by Kielblock et al. will lead to all oscillators receiving identical input at all times. Order conservation is ensured, as will be discussed in detail in the discussion section.

Often in larger networks the effect of self-loops is assumed to be negligible due to their small contribution relative to the input from the rest of the network or found to not having a substantial effect [45].

However, here we present a case where the presence of self-loops leads to qualitatively different behavior.

Consequently, removing the self-loops gradually has to lead to a transition from a 
system of order conservation to a system with oscillators overtaking each other.

Transitions from one state to another are fundamental phenomena in nature. E.g. transitions from aggregate states in thermodynamics such as transitions from solid to liquid or gaseous state shape our environment. These transitions can occur discontinuous (e.g. the transition from liquid to gaseous state induced by heating produces a sudden increase in density) or continuous (e.g. in the ferromagnetic transition). For many applications it is vital to understand the nature of a certain transition and to determine critical parameters for a transition to occur. Examples include determining whether an epidemic will spread [26, whether a material will be stable or whether queues will grow indefinitely. There are different fields that study transitions analytically, e.g. percolation theory or bifurcation theory. Often transitions occur by changing a macroscopic control parameter in an otherwise unstructured/symmetrical system (e.g. changing the temperature in the Ising model or changing the connection probability in a percolation network).

First we demonstrate numerically that the transition from order conservation to breaking of order conservation exists in an all-to-all pulse-coupled oscillator network, where pulse reception does not lead to any non-smoothness in the trajectories. Then we study the transition analytically in a system with delayed $\delta$-pulses and uncover the mechanisms behind the reordering process, which ranges from simple reordering patterns to quasi-chaotic reordering.

\section{The Structure of This Chapter}

This chapter is structured in the following way: In the second section we consider a smooth pulse-coupled system that shows reordering and demonstrate that introducing self-loops leads to order conservation. In the third section we introduce a $\delta$-pulsecoupled system, that allows for analytic treatment. We derive conditions for order conservation in the parameter space including self-loop strength and describe the reordering process analytically. Within the reordering parameter regime we observe different patterns of reordering including chaos-like mixing. In the fourth and final part we provide a brief summary and discuss the results from a dynamical system's perspective, focusing on the dynamical features of pulse-coupling and self-loops.

\subsection{Breaking of Order Conservation by Removing Self-Loops in a Smooth Pulse-Coupled System}

While Golubitsky et al. 25] showed that a symmetric all-to-all coupled network described by ODEs necessarily shows order conservation, Kielblock et al. [38] presented an example of a symmetric all-to-all pulse-coupled system with breaking of order conservation. Since the pulse-coupling introduced by Kielblock et al. had no delay and did not induce any non-smoothness into the system, the dynamical feature of pulsecoupling itself seems to be responsible for the breaking of order conservation. Here we introduce self-loops into the system studied by Kielblock et al. and find, that the system with self-loops returns to order conservation. 


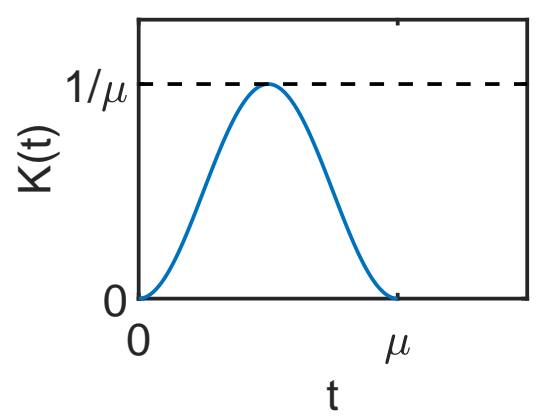

Figure 4.1: Coupling kernel $K$. The coupling kernel is constructed to induce no non-smoothness, when a pulse is received.

\subsubsection{System Definition}

The network consists of $N=4$ all-to-all coupled nodes with the following dynamics:

$$
\begin{aligned}
& \dot{x}_{i}=f\left(x_{i}\right)+\sum_{j=1}^{N} \sum_{s \in \mathbb{Z}} \varepsilon_{i j} K\left(t-t_{j}^{s}\right) \\
& x_{i}\left(t_{i}^{s-}\right)=1 \rightarrow x_{i}\left(t_{i}^{s+}\right)=0
\end{aligned}
$$

$f\left(x_{i}\right)=I-x_{i}$ with $I=1.1$ captures the intrinsic dynamics of each node $i$, which is oscillatory, since the free dynamics eventually reach the threshold $x_{i}\left(t_{i}^{s-}\right)=1$, the oscillator sends a spike and resets to $x_{i}\left(t_{i}^{s+}\right)=0 . t_{i}^{s}$ indicates the sending time of the sth spike of node $i$. $\varepsilon$ is the coupling strength between nodes with $\varepsilon=-1$ if $i \neq j$. If $i=j, \varepsilon=0$ in the system without self-loops and $\varepsilon=1$ in the system with self-loops. The coupling kernel $K$ is given by

$$
K(t)=\frac{1}{\mu} \sin ^{2}\left(\frac{\pi}{\mu} t\right)
$$

for $t \in[0, \mu]$ and $K(t)=0$ otherwise (see figure 4.1). The kernel is constructed such that the dynamics are differentiable (except for the oscillator reset). The initial conditions were chosen close to the synchronous state.

\subsubsection{Transition to Order Conservation with Self-Loops}

In the original system without self-coupling the oscillators pass each other, while order is conserved in the system with self-coupling, see figure 4.2. Therefore if we remove self-loops by gradually decreasing their strength we expect to observe a transition from order conservation to breaking of order conservation. In the next section we analyse this transition and the reordering process in case of breaking of order conservation in greater detail. Since the smooth system presented here is not analytically tractable, we will resort to studying the transition in a simpler system, that is analytically tractable. That system is characterized by delayed $\delta$-pulse-coupling instead of a smooth coupling kernel. The analysis is based on the approach presented by Kielblock et al. 38. 

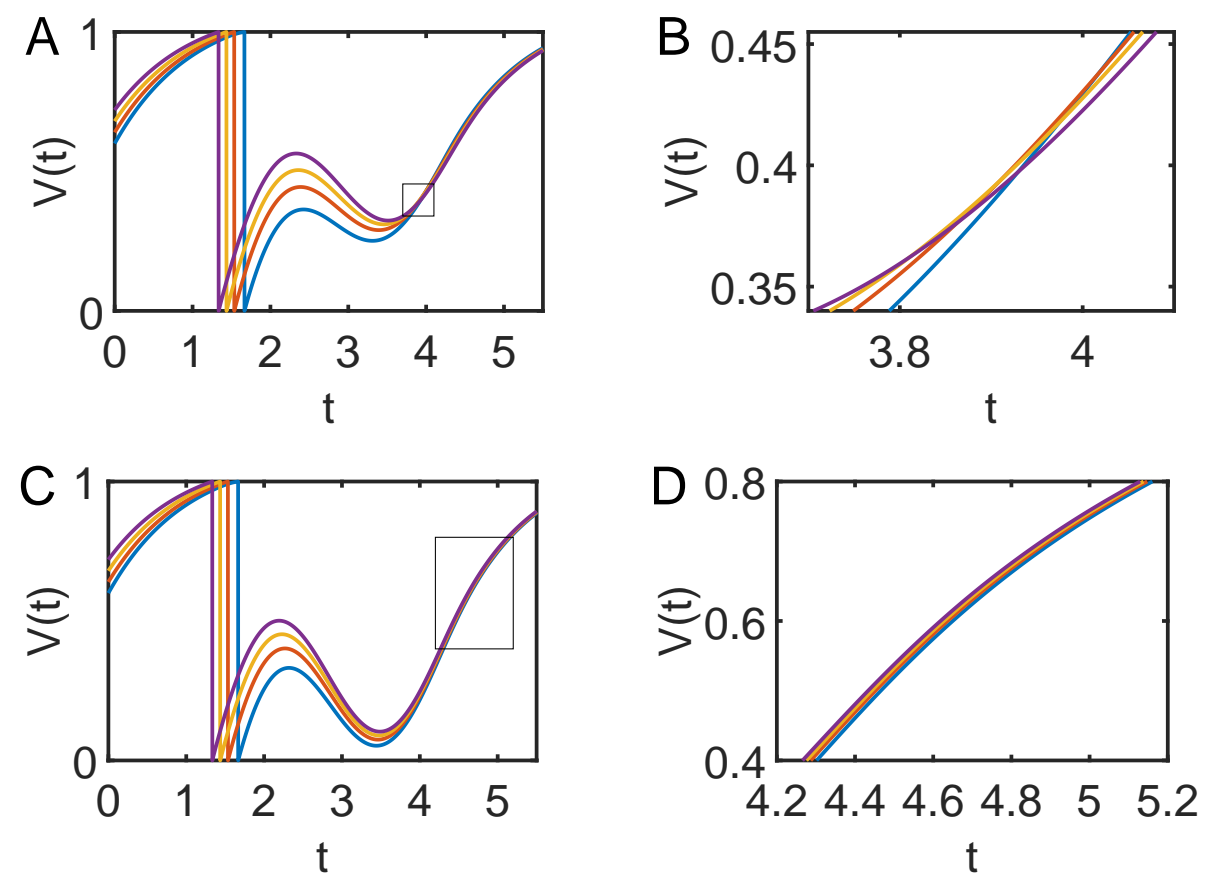

Figure 4.2: Transition from overtaking to order conservation in a symmetric smooth pulse-coupled system by introducing self-loops. Panel $\mathrm{A}$ and $\mathrm{B}$ show trajectories of the 4-oscillator system with no self-loops studied by Kielblock et al. [38. Panel C and D show trajectories of the same system with self-loops. Panel B and D show magnified regions from panel $\mathrm{A}$ and $\mathrm{C}$, respectively. The initial conditions were chosen close to the synchronous state. With no self-coupling the oscillators pass each other, while with self-coupling their order is preserved. 


\subsection{Analysis of Reordering Processes in a Spiking System with Self-Loops}

\subsubsection{System Definition}

System class and phase representation Here we consider $\delta$-pulse-coupled networks with $N$ nodes of the following form:

$$
\begin{aligned}
& \frac{\mathrm{d} x_{i}}{\mathrm{~d} \hat{t}}=f\left(x_{i}\right)+\sum_{j=1}^{N} \sum_{s} \varepsilon_{i j} K\left(\hat{t}-\hat{t}_{j}^{s}\right) \\
& x_{i}\left(\hat{t}_{i}^{s-}\right)=1 \rightarrow x_{i}\left(\hat{t}_{i}^{s+}\right)=0 .
\end{aligned}
$$

$f(x)$ captures the intrinsic dynamics of each node. We assume $f(x)$ to be smooth. The free dynamics without input starting at the reset point $x=0$ are assumed to reach threshold eventually at $x=1$, leading to a spike generation and reset and an overall oscillatory behavior. Reset and threshold are chosen to be at 0 and 1 . Systems with other reset and threshold can be easily rescaled accordingly. $\hat{t}_{i}^{s}$ indicates the sending time of the $s$ th spike of node $i . K\left(\hat{t}-\hat{t}_{j}^{s}\right)=\delta\left(\hat{t}-\hat{t}_{j}^{s}-\hat{\tau}\right)$ is the coupling kernel, receiving a spike leads to an instantaneous change in the variable $x_{i}$. $\hat{\tau}$ denotes a constant delay. $\varepsilon_{i j}$ is the coupling strength from node $j$ to node $i$. We denote the solution to the free evolution of an oscillator starting at $x(0)=0$ with $F(\hat{t})$. The time $\hat{t}$ until threshold crossing is $T: F(T)=1 . F(\hat{t})$ is monotonically increasing, $F^{\prime}>0$. (Due to the existence and uniqueness theorem trajectories need to be continuous and flow is unique. The trajectory in a one dimensional phase space therefore can only proceed in one direction or approach a fixed point but it cannot return).

Equation 4.1 can be translated into a phase representation. We introduce the phase variable $\phi \in[0,1]$ with the following time evolution:

$$
\begin{aligned}
& \dot{\phi}_{i}=1+Z_{\varepsilon_{i j}}\left(\phi_{i}\right) \sum_{j=1}^{N} \sum_{s} \delta\left(t-t_{j}^{s}-\tau\right) \\
& \phi_{i}\left(t_{i}^{s-}\right)=1 \rightarrow \phi_{i}\left(t_{i}^{s+}\right)=0 .
\end{aligned}
$$

Hence, the free solution of the phase variable $\phi$ needs one time unit $t$ from reset $(\phi=0)$ to reach threshold $(\phi=1)$. Therefore we rescale the old time $\hat{t}$ with the factor $1 / T$ to achieve correspondence. The delay is rescaled: $\tau=\hat{\tau} / T$. The phase response curve $Z_{\varepsilon_{i j}}(\phi)$ provides the size of the instantaneous jump in phase of the receiving oscillator induced by the reception of a spike and depends on the current phase $\phi_{i}$ and the coupling strength $\varepsilon_{i j}$.

To achieve correspondence $Z_{\varepsilon_{i j}}(\phi)$ is constructed in the following way. First we determine the phase after spike reception $\phi_{\text {post }}$ depending the phase directly before spike reception $\phi_{\text {pre }}$

$$
\phi_{\text {post }}=U^{-1}\left(U\left(\phi_{\text {pre }}\right)+\varepsilon_{i j}\right)=H_{\varepsilon_{i j}}\left(\phi_{\text {pre }}\right) .
$$

$U(\phi)$ is obtained by rescaling the free solution $F(\hat{t}): U:(-\infty, 1) \rightarrow(-\infty, 1) ; \phi \rightarrow$ $U(\phi):=F(\phi T), U(0)=0$ and $U(1)=1$. Since $F^{\prime}>0$ also $U^{\prime}>0 . U(\phi)$ is called rise function, $U^{-1}(x)$ denotes the inverse rise function. $H_{\varepsilon_{i j}}(\phi)$ is called interaction 

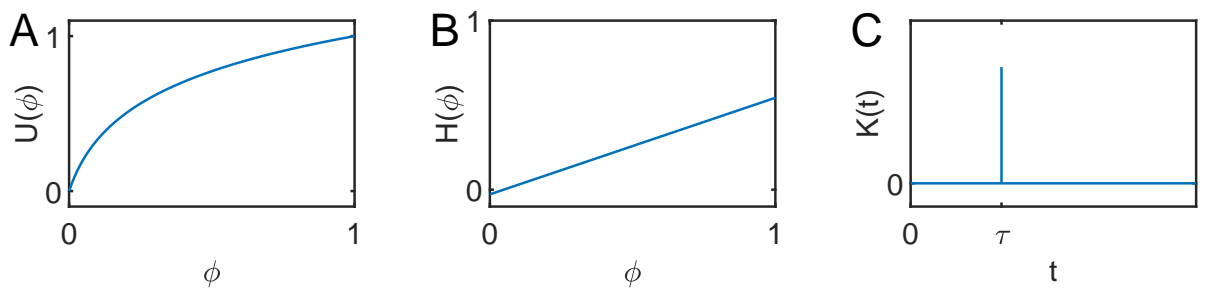

Figure 4.3: Rise function $U_{b}$, interaction function $H$ and coupling kernel $K$. Panel A shows the strictly monotonically increasing rise function. The parameter $b$ controls the bending. The considered class of rise functions $U_{b}$ leads to an affine interaction function $H$ (panel B). Panel $\mathrm{C}$ shows the coupling kernel $K(t)=\delta(t-\tau)$. Parameters: $b=2.8, \varepsilon=-0.2$.

function. The phase response curve $Z_{\varepsilon_{i j}}(\phi)$ and the interaction function $H_{\varepsilon_{i j}}(\phi)$ are related through the following equation:

$$
Z_{\varepsilon_{i j}}\left(\phi_{\text {pre }}\right)=\phi_{\text {post }}-\phi_{\text {pre }}=H_{\varepsilon_{i j}}\left(\phi_{\text {pre }}\right)-\phi_{\text {pre }}
$$

Rise function and interaction function. We consider a system of coupled phase oscillators according to equations 4.2 4.3 and 4.4. We choose a specific class of rise function (see figure 4.3 , panel A):

$$
U_{b}(\phi)=\frac{1}{b} \ln \left[1+\left(e^{b}-1\right) \phi\right] .
$$

The parameter $b>0$ controls the concavity of the curve, larger $b$ correspond to stronger bending. The inverse rise function is given by

$$
U_{b}^{-1}(x)=\phi=\frac{e^{b x}-1}{e^{b}-1}
$$

The interaction function $H_{\varepsilon_{i j}}(\phi)$ is provided by

$$
\begin{aligned}
H_{\varepsilon_{i j}}(\phi) & =U_{b}^{-1}\left(U_{b}(\phi)+\varepsilon_{i j}\right) \\
& =\frac{e^{b \varepsilon_{i j}}-1}{e^{b}-1}+e^{b \varepsilon_{i j}} \phi,
\end{aligned}
$$

see figure 4.3 , panel $\mathrm{B}$. The derivative with respect to $\phi$ is given by

$$
\frac{\mathrm{d} H_{\varepsilon_{i j}}(\phi)}{\mathrm{d} \phi}=H_{\varepsilon_{i j}}^{\prime}(\phi)=e^{b \varepsilon_{i j}}
$$

Due to the choice of a specific class of rise functions $U \varepsilon_{i j}(\phi)$, the interaction function $H_{\varepsilon_{i j}}(\phi)$ is affine and the slope $H_{\varepsilon_{i j}}^{\prime}$ is independent of its argument $\phi$ :

$$
H_{\varepsilon_{i j}}^{\prime}(\phi)=H_{\varepsilon_{i j}}^{\prime}
$$


Coupling strength. We are interested in varying the self-loop strength in a permutationsymmetric system. Therefore, $\varepsilon_{i j}=\varepsilon$ if $i \neq j$ denotes the coupling strength between two different nodes, while $\varepsilon_{i j}=\sigma$ if $i=j$ denotes the self-loops strength, $\alpha \in[0,1]$ with $\alpha \varepsilon=\sigma$ captures the relative strength of self-loops to the coupling strength between nodes in the network.

Assumption of blocked spike sending and reception times. We assume that the system is in a state with spike sending events of all oscillators occurring as a block followed by a block of spike reception events of all oscillators. Such a situation is found in the vicinity of the synchronous state defined by all oscillators having identical phases at all times.

Replacing the phase vector by a phase difference vector. Without loss of generality we can label the oscillators of an N-dimensional system according to their ordering in phases relative to each other directly after all oscillators have sent their spikes:

$$
1>\phi_{1}>\phi_{2}>\ldots>\phi_{N}>0 \text {. }
$$

This system of oscillators can equally well be described by giving the phase of the first oscillator and the differences in phases of the other oscillators: Let $\Delta_{i j}=\phi_{i}-\phi_{j}$ be the difference in phase between oscillator $i$ and $j$. Now the system can be described by the following coordinates $\left\{\phi_{1}, \Delta_{1,2}, \Delta_{2,3}, \ldots, \Delta_{N-1, N}\right\}$.

We assume that the first oscillator sends its spike at $t=0$, the second oscillator at $t=\Delta_{1,2}$ and so on, such that the $i^{\text {th }}$ oscillator will send its spike at $t=\sum_{j=1}^{i-1} \Delta_{k, k+1}$. The pulse of the first oscillator will then be received by all oscillators at $t=\tau$, the next spike of the second oscillator will be received at $t=\tau+\Delta_{1,2}$ and so on, such that the pulse sent by the $i^{\text {th }}$ oscillator will be received at $t=\tau+\sum_{j=1}^{i-1} \Delta_{k, k+1}$.

\subsubsection{Return Map for Phase Differences}

A mapping from phases before to after reception of all blocked spikes. For notational reasons we define the following function of a phase shift:

$$
S_{\Delta}(\phi)=\phi+\Delta .
$$

It is possible to express the phases of oscillator $i$ and $i+1$ after reception of all spikes at time $\tau+\Delta_{1, N}$, given their phases just before the first spike is received at time $\tau$. The effect of the full sequence of alternating spike receptions and phase shifts between spikes is aggregated in the following function composition: 


$$
\begin{aligned}
\phi_{i}\left(\tau+\Delta_{1, N}\right)= & H_{\varepsilon} \circ S_{\Delta_{N-1, N}} \circ \cdots \circ H_{\varepsilon} \circ S_{\Delta_{i+1, i+2}} \circ H_{\varepsilon} \circ S_{\Delta_{i, i+1}} \circ H_{\sigma} \circ S_{\Delta_{i-1, i}} \circ \ldots \\
& \cdots \circ S_{\Delta_{2,3}} \circ H_{\varepsilon} \circ S_{\Delta_{1,2}} \circ H_{\varepsilon}\left(\phi_{i}(\tau)\right)
\end{aligned}
$$

This equation is solved by untangling the functions step by step applying $H_{\varepsilon}(a+b)=$ $H \varepsilon(a)+H_{\varepsilon}^{\prime}(a) \cdot b$ repeatedly. Also note that $H_{\alpha}\left(H_{\beta}(a)\right)=H_{\alpha+\beta}(a)$.

$$
H_{\varepsilon}\left(\phi_{i}(\tau)\right)=H_{\varepsilon}\left(\tau-\sum_{k=1}^{i-1} \Delta_{k, k+1}\right)=H_{\varepsilon}(\tau)+H_{\varepsilon}^{\prime}(\tau) \cdot\left(-\sum_{k=1}^{i-1} \Delta_{k, k+1}\right)
$$

$$
\begin{aligned}
H_{\varepsilon} \circ S_{\Delta_{1,2}} \circ H_{\varepsilon}\left(\phi_{i}(\tau)\right) & \left.=H_{\varepsilon} \circ\left[H_{\varepsilon}(\tau)+H_{\varepsilon}^{\prime}(\tau)\right) \cdot\left(-\sum_{k=1}^{i-1} \Delta_{k, k+1}\right)+\Delta_{1,2}\right] \\
& =H_{2 \varepsilon}(\tau)+H_{\varepsilon}^{\prime}\left(H_{\varepsilon}(\tau)\right) \cdot\left(-\sum_{k=1}^{i-1} \Delta_{k, k+1}\right)+H_{\varepsilon}^{\prime}\left(H_{\varepsilon}(\tau)\right) \cdot \Delta_{1,2}
\end{aligned}
$$

Untangling the complete function composition using equation 4.5 leads to:

$$
\begin{aligned}
\phi_{i}(\tau+ & \left.\Delta_{1, N}\right)=H_{(N-1) \varepsilon+\sigma}(\tau) \\
& +H_{\varepsilon}^{\prime}\left(H_{(N-2) \varepsilon+\sigma}(\tau)\right) \cdot H_{\varepsilon}^{\prime}\left(H_{(N-3) \varepsilon+\sigma}(\tau)\right) \ldots H_{\varepsilon}^{\prime}\left(H_{(i-1) \varepsilon+\sigma}(\tau)\right) \\
& \left.\cdot H_{\sigma}^{\prime}\left(H_{(i-1) \varepsilon}(\tau)\right) \cdot H_{\varepsilon}^{\prime}\left(H_{(i-2) \varepsilon}(\tau)\right) \ldots H_{\varepsilon}^{\prime}\left(H_{\varepsilon}(\tau)\right) \cdot H_{\varepsilon}^{\prime}(\tau)\right) \cdot\left(-\sum_{k=1}^{i-1} \Delta_{k, k+1}\right) \\
& +H_{\varepsilon}^{\prime}\left(H_{(N-2) \varepsilon+\sigma}(\tau)\right) \cdot H_{\varepsilon}^{\prime}\left(H_{(N-3) \varepsilon+\sigma}(\tau)\right) \ldots H_{\varepsilon}^{\prime}\left(H_{(i-1) \varepsilon+\sigma}(\tau)\right) \\
& \cdot H_{\sigma}^{\prime}\left(H_{(i-1) \varepsilon}(\tau)\right) \cdot H_{\varepsilon}^{\prime}\left(H_{(i-2) \varepsilon}(\tau)\right) \ldots H_{\varepsilon}^{\prime}\left(H_{\varepsilon}(\tau)\right) \cdot \Delta_{1,2} \\
& \vdots \\
& +H_{\varepsilon}^{\prime}\left(H_{(N-2) \varepsilon+\sigma}(\tau)\right) \cdot H_{\varepsilon}^{\prime}\left(H_{(N-3) \varepsilon+\sigma}(\tau)\right) \ldots H_{\varepsilon}^{\prime}\left(H_{(i-1) \varepsilon+\sigma}(\tau)\right) \\
& \cdot H_{\sigma}^{\prime}\left(H_{(i-1) \varepsilon}(\tau)\right) \cdot H_{\varepsilon}^{\prime}\left(H_{(i-2) \varepsilon}(\tau)\right) \cdot \Delta_{i-2, i-1} \\
& +H_{\varepsilon}^{\prime}\left(H_{(N-2) \varepsilon+\sigma}(\tau)\right) \cdot H_{\varepsilon}^{\prime}\left(H_{(N-3) \varepsilon+\sigma}(\tau)\right) \ldots H_{\varepsilon}^{\prime}\left(H_{(i-1) \varepsilon+\sigma}(\tau)\right) \\
& \cdot H_{\sigma}^{\prime}\left(H_{(i-1) \varepsilon}(\tau)\right) \cdot \Delta_{i-1, i} \\
& +H_{\varepsilon}^{\prime}\left(H_{(N-2) \varepsilon+\sigma}(\tau)\right) \cdot H_{\varepsilon}^{\prime}\left(H_{(N-3) \varepsilon+\sigma}(\tau)\right) \ldots H_{\varepsilon}^{\prime}\left(H_{(i-1) \varepsilon+\sigma}(\tau)\right) \\
& \cdot \Delta_{i, i+1} \\
& \vdots \\
& +H_{\varepsilon}^{\prime}\left(H_{(N-2) \varepsilon+\sigma}(\tau)\right) \cdot H_{\varepsilon}^{\prime}\left(H_{(N-3) \varepsilon+\sigma}(\tau)\right) \cdot \Delta_{N-2, N-1} \\
& +H_{\varepsilon}^{\prime}\left(H_{(N-2) \varepsilon+\sigma}(\tau)\right) \cdot \Delta_{N-1, N}
\end{aligned}
$$




$$
\begin{aligned}
= & H_{(N-1) \varepsilon+\sigma}(\tau) \\
& +H_{\varepsilon}^{\prime} \cdot H_{\varepsilon}^{\prime} \ldots H_{\varepsilon}^{\prime} \cdot H_{\sigma}^{\prime} \cdot H_{\varepsilon}^{\prime} \ldots H_{\varepsilon}^{\prime} \cdot H_{\varepsilon}^{\prime} \cdot\left(-\sum_{k=1}^{i-1} \Delta_{k, k+1}\right) \\
& +H_{\varepsilon}^{\prime} \cdot H_{\varepsilon}^{\prime} \ldots H_{\varepsilon}^{\prime} \cdot H_{\sigma}^{\prime} \cdot H_{\varepsilon}^{\prime} \ldots H_{\varepsilon}^{\prime} \cdot \Delta_{1,2} \\
& \vdots \\
& +H_{\varepsilon}^{\prime} \cdot H_{\varepsilon}^{\prime} \ldots H_{\varepsilon}^{\prime} \cdot H_{\sigma}^{\prime} \cdot H_{\varepsilon}^{\prime} \cdot \Delta_{i-2, i-1} \\
& +H_{\varepsilon}^{\prime} \cdot H_{\varepsilon}^{\prime} \ldots H_{\varepsilon}^{\prime} \cdot H_{\sigma}^{\prime} \cdot \Delta_{i-1, i} \\
& +H_{\varepsilon}^{\prime} \cdot H_{\varepsilon}^{\prime} \ldots H_{\varepsilon}^{\prime} \cdot \Delta_{i, i+1} \\
& \vdots \\
& +H_{\varepsilon}^{\prime} \cdot H_{\varepsilon}^{\prime} \ldots \Delta_{N-2, N-1} \\
& +H_{\varepsilon}^{\prime} \cdot \Delta_{N-1, N} \\
= & H_{(N-1) \varepsilon+\sigma}(\tau) \\
& +H_{\varepsilon}^{\prime N-1} \cdot H_{\sigma}^{\prime} \cdot\left(-\sum_{k=1}^{i-1} \Delta_{k, k+1}\right)+H_{\varepsilon}^{\prime N-2} \cdot H_{\sigma}^{\prime} \cdot \Delta_{1,2} \ldots \\
& +H_{\varepsilon}^{\prime N-i+1} \cdot H_{\sigma}^{\prime} \cdot \Delta_{i-2, i-1}+H_{\varepsilon}^{\prime N-i} \cdot H_{\sigma}^{\prime} \cdot \Delta_{i-1, i}+H_{\varepsilon}^{\prime N-i} \cdot \Delta_{i, i+1} \ldots \\
& +H_{\varepsilon}^{\prime 2} \cdot \Delta_{N-2, N-1}+H_{\varepsilon}^{\prime} \cdot \Delta_{N-1, N}
\end{aligned}
$$

A return map for the phase difference vector. Now we consider the phase difference vector between neighboring oscillators: $\vec{\Delta}$. We define $F_{\tau, \varepsilon, \sigma}(\Delta)$ as the map from before to after the $k$ th block of spike reception.

$$
\vec{\Delta}\left(\tau+\Delta_{1, N}\right)=F_{\tau, \varepsilon, \sigma}\left(\vec{\Delta}\left(\tau^{-}\right)\right)
$$

Outside the block of spike receptions all phases proceed with speed 1 (equation 4.2). Further, $\vec{\Delta}$ is conserved across the block of phase resets. Therefore, the map $F$ is the return map from before the $k$ th block of spike reception to before the $k+1$ th block of spike reception: $\vec{\Delta}^{r}=F_{\tau, \varepsilon, \sigma}(\vec{\Delta})$ :

$$
\begin{aligned}
\Delta_{i, i+1}^{r} & =\phi_{i}\left(\tau+\Delta_{1, N}\right)-\phi_{i+1}\left(\tau+\Delta_{1, N}\right) \\
& =\left[H_{\varepsilon}^{\prime N-i}-H_{\varepsilon}^{\prime N-i-1} \cdot H_{\sigma}^{\prime}+H_{\varepsilon}^{\prime N-1} \cdot H_{\sigma}^{\prime}\right] \Delta_{i, i+1} \\
& =\left[\left(H_{\varepsilon}^{\prime}-H_{\sigma}^{\prime}\right) H_{\varepsilon}^{\prime N-1-i}+H_{\varepsilon}^{\prime N-1} H_{\sigma}^{\prime}\right] \Delta_{i, i+1} \\
& =\left[\left(e^{\varepsilon b}-e^{\alpha \varepsilon b}\right) e^{\varepsilon b^{N-1-i}}+e^{\varepsilon b^{N-1}} e^{\alpha \varepsilon b}\right] \Delta_{i, i+1} \\
& =\lambda_{i} \Delta_{i, i+1}
\end{aligned}
$$

Interestingly, the return map for each interval $\Delta_{i, i+1}$ of $\vec{\Delta}$ only depends on itself. The return map $F$ is a diagonal matrix with diagonal elements $\lambda_{i}$ as eigenvalues. $F$ is independent of $\tau$. 

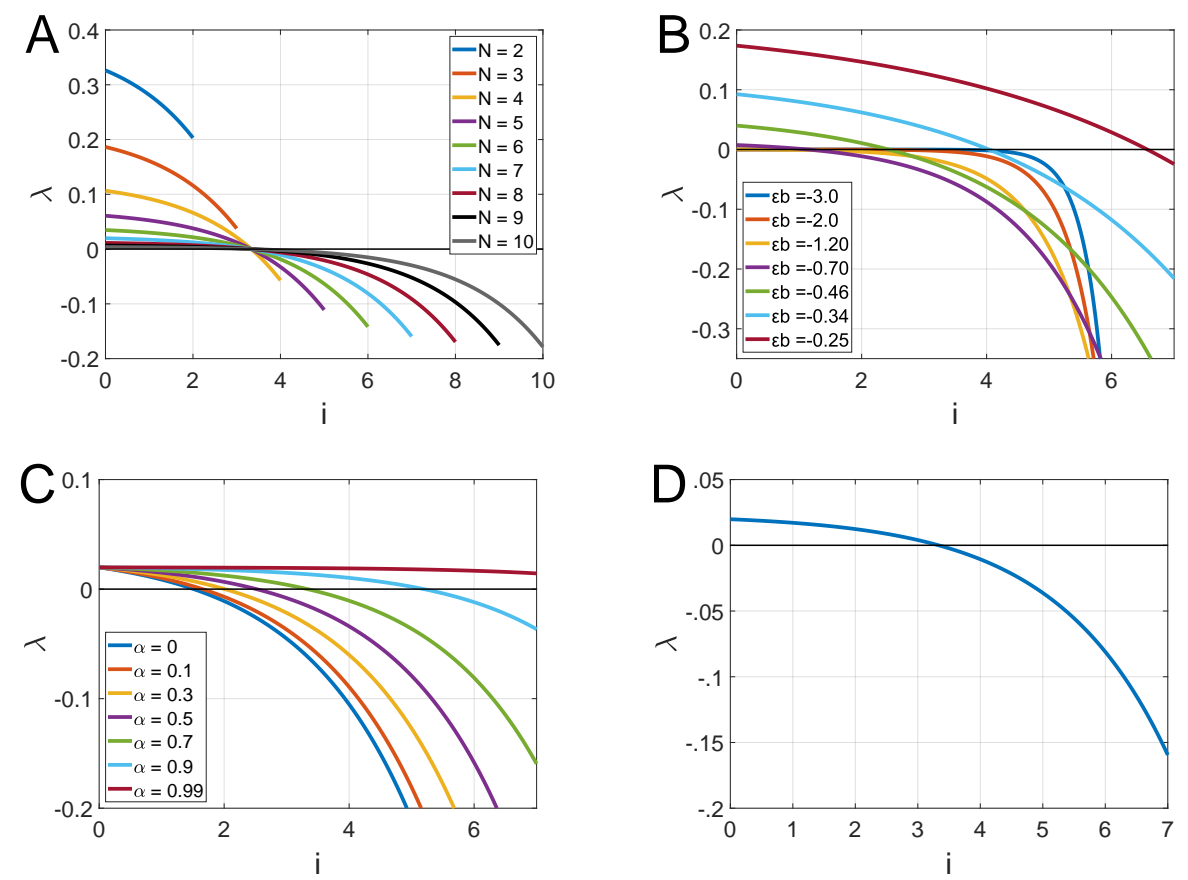

Figure 4.4: Dependence of $\boldsymbol{\lambda}_{\boldsymbol{i}}$ on different parameters. Panel A: Dependence on the system size $N$. If the curves have a zero crossing, it is identical. Panel B: Dependence on $\varepsilon b$, the parameters occur always together and are considered together. Panel C: Dependence on the self-loop strength $\alpha$. Panel D: $\lambda(i)$ depending on default parameters. Default parameters (see main text) are used in all four panels, if not explicitly noted otherwise. 


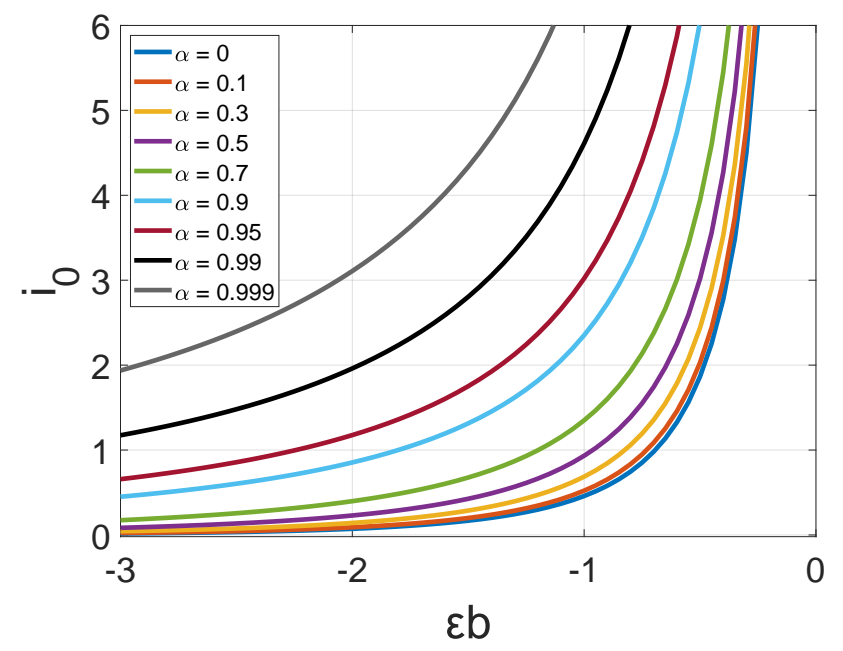

Figure 4.5: The zero crossing $i_{0}$ of $\lambda(i)$ depending on parameters. The curve wit $\alpha=0$ appears to be a limiting curve: $\left.i_{0}\right|_{\alpha=0}(b \varepsilon)=\frac{\ln \left[1-e^{b \varepsilon}\right]}{b \varepsilon}$. The bending point is determined via $i_{\text {bend }}=\left\lceil i_{0}\right\rceil$.

Exploring the eigenvalue $\lambda_{i}$. For the remainder of the chapter we assume negative coupling: $\varepsilon<0$. Timme et al. [83, 84, 86] have shown that the synchronous state $\left(\phi_{i}(t)=\phi_{j}(t)\right.$ for all oscillators and all times $\left.t\right)$ in such a system exists and is stable. We will use $\varepsilon=-0.2, b=2.8, \alpha=0.7$ and $N=7$ as default parameter set (see figure 4.4 panel $\mathrm{D}$ ).

figure 4.4 shows how the eigenvalues $\lambda_{i}$ interpreted as a function $\lambda(i)$ change with different parameters. The interval with index $i$ corresponds to the phase difference interval $\Delta_{i, i+1}$.

The intercept of $\lambda(i)$ is positive:

$$
\lambda(0)=\left(e^{\varepsilon b}-e^{\alpha \varepsilon b}\right) e^{\varepsilon b^{N-1}}+e^{\varepsilon b^{N-1}} e^{\alpha \varepsilon b}=e^{N \varepsilon b}>0
$$

$\lambda(i)$ is monotonically decreasing:

$$
\frac{\mathrm{d} \lambda(i)}{\mathrm{d} i}=\left(e^{\varepsilon b}-e^{\alpha \varepsilon b}\right) e^{\varepsilon b^{N-1-i}}(-\varepsilon b)<0
$$

and bend concave down:

$$
\frac{\mathrm{d} \lambda(i)^{2}}{\mathrm{~d}^{2} i}=\left(e^{\varepsilon b}-e^{\alpha \varepsilon b}\right) e^{\varepsilon b^{N-1-i}}(-\varepsilon b)^{2}<0
$$

$\lambda(i)$ is bounded: $|\lambda(i)| \leq 1$ for $i \in[0, N-1]$ :

$\varepsilon b \leq \sigma b=\alpha \varepsilon b \leq 0$. It follows that $-1<\left(e^{\varepsilon b}-e^{\alpha \varepsilon b}\right) \leq 0$ and $0<e^{\varepsilon b^{N-1-i}} \leq 1$ and $0<e^{\varepsilon b^{N-1}} e^{\alpha \varepsilon b} \leq 1$. Therefore $-1<\lambda(i)=\left(e^{\varepsilon b}-e^{\alpha \varepsilon b}\right) e^{\varepsilon b^{N-1-i}}+e^{\varepsilon b^{N-1}} e^{\alpha \varepsilon b} \leq 1$. 


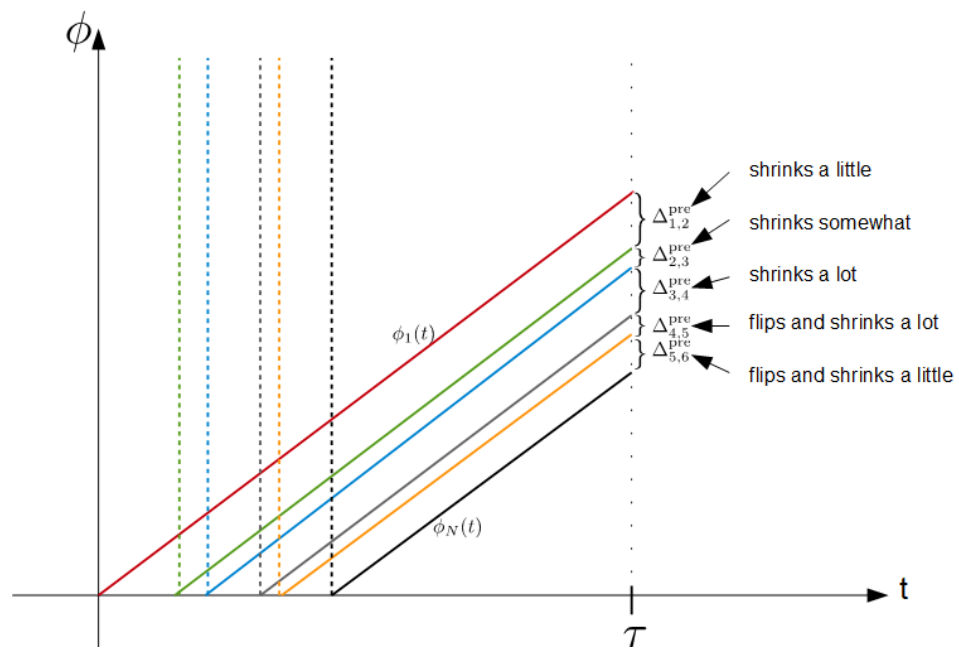

Figure 4.6: A sketch of how intervals change across a block of spike reception. $\lambda_{i}$ determines how each interval between two neighboring oscillators $\boldsymbol{\Delta}_{i, i+1}$ changes size and potentially flips sign.

Zero crossing $\lambda\left(\boldsymbol{i}_{\mathbf{0}}\right)=\mathbf{0}$. Since $\lambda(i)$ is monotonically decreasing (equation 4.7) and starts above zero (equation 4.6) there is exactly one zero crossing $i_{0}$, see figure 4.5 . However, it may lie outside of the range of interest, that is $i_{0} \geq N-1$.

$$
\begin{gathered}
0=\lambda\left(i_{0}\right)=\left(e^{\varepsilon b}-e^{\alpha \varepsilon b}\right) e^{\varepsilon b^{N-1-i_{0}}}+e^{\varepsilon b^{N-1}} e^{\alpha \varepsilon b} \\
\Longleftrightarrow 0=\left(e^{b \varepsilon}-e^{b \alpha \varepsilon}\right)\left[e^{b \varepsilon}\right]^{-i_{0}}+e^{b \alpha \varepsilon} \\
i_{0}=\frac{\ln \left[e^{b \alpha \varepsilon}-e^{b \varepsilon}\right]}{b \varepsilon}-\alpha
\end{gathered}
$$

$i_{0}$ does not depend on the system size $N$ nor does it depend on the phases or phase differences between oscillators. For $i_{0}$ depending on parameters see figure 4.5 .

\subsubsection{A Folding Mechanism}

$\lambda_{i}$ determines how the interval $\Delta_{i, i+1}$ changes through the completion of a cycle. Therefore the reordering process over one cycle is fully determined by the eigenvalues $\lambda_{i}$. For a fixed parameter set the change of each interval only depends on its index $i$ and not on the phases or phase differences between oscillators.

For some parameters $\lambda(i)$ has a zero-crossing $i_{0}\left(\lambda\left(i_{0}\right)=0\right)$ within the interval ]1, $N-1$ [. All intervals with indices $i>i_{0}$ change their sign and the corresponding oscillators change their ordering, while all intervals with $i<i_{0}$ keep their sign and oscillators their ordering. The smaller $\left|i-i_{0}\right|$, the smaller $\left|\lambda_{i}\right|$ and the stronger the shrinking of the interval. Figure 4.6 sketches the qualitative behavior assuming that the zero crossing $i_{0}$ is situated between 3 and $4\left(3<i_{0}<4\right)$.

The reordering mechanism can be illustrated via analogy to a system of beads on a rubber string, see figure 4.7. Each bead corresponds to the phase of an oscillator. The spaces between the beads correspond to the phase differences $\Delta_{i, i+1}$. The block of spike receptions induces a folding of the string and a shrinking of the distances 

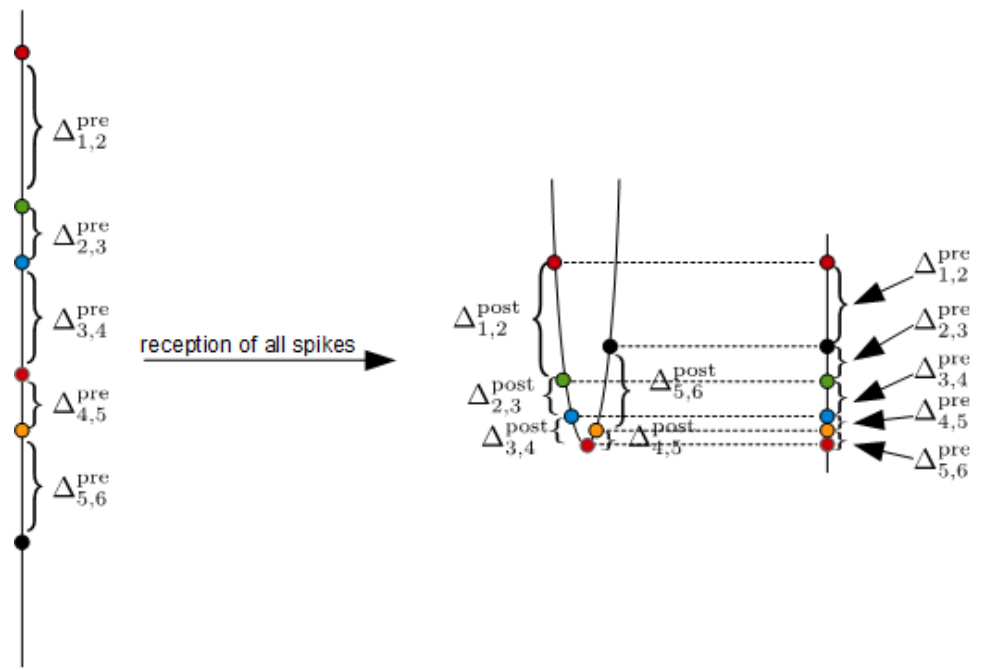

Figure 4.7: The folding mechanism resembles folding a rubber string with beads. In the displayed sketch the bending point is the 4 th bead, the 5 th and 6th bead are folded up and are nested between beads 1 to 4 . Intervals closed to the 4 th bead show stronger shrinking that intervals further away. After the folding is complete oscillators corresponding to the beads and intervals are renamed according to the new ordering.

between the beads. Neighboring beads are connected with rubber bands of different strength, determined by $\lambda_{i}$, resulting in different shrinking factors.

The folding occurs at one specific bead, we will call bending point, which becomes the minimum of the folded string. The bending point is always the closest integer larger than the the zero crossing $\left(i_{\text {bend }}=\left\lceil i_{0}\right\rceil\right)$ or $N$ in case $\left\lceil i_{0}\right\rceil>N$. All beads below the bending point are are flipped up, the distances between all beads shrink according to $\lambda_{i}$. After bending the upfolded beads are nested between the others, resulting in a reordering. This reordering depends on the previous intervals $\vec{\Delta}$ as well as $\vec{\lambda}$, which depends on the system's parameters.

$\Delta_{i, i+1}^{r}=\lambda_{i} \Delta_{i, i+1}$ provides the distance between the initially neighboring oscillators $i$ and $i+1$ after spike reception. However, the two oscillators may not be neighboring any more, due to the reordering induced by the folding procedure. A full analytic description of the reordering mechanism should provide a phase difference vector of the oscillators after spike reception according to the new neighborhood relations depending on the phase difference vector of the previously neighboring oscillators before spike reception. This is achieved in the following way:

Relabeling and the new phase difference vector $\boldsymbol{\Delta}^{\vec{n} \text { ew }}$. Oscillator 1 is taken as reference. Since we only consider relative phases, we arbitrarily set the phase of oscillator 1 after the block of spike receptions to $\phi_{1}^{r}=0$. The phase distance relative 

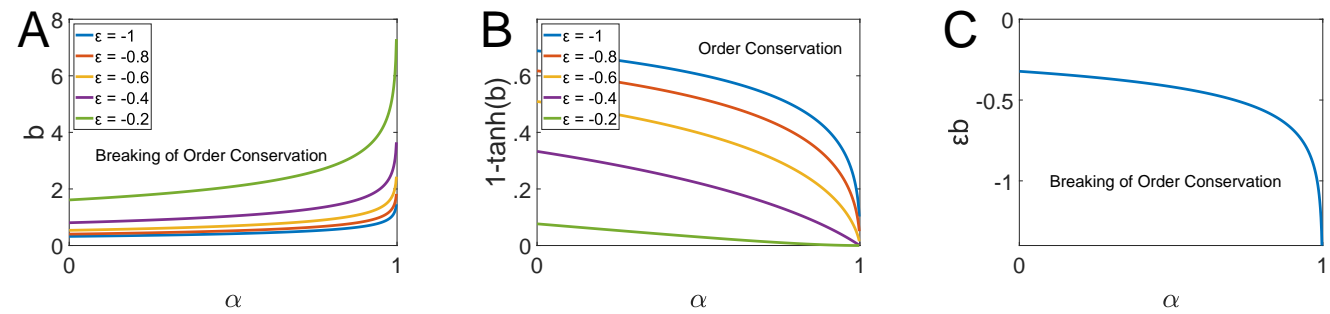

Figure 4.8: Transition from overtaking to order conservation by increasing the relative self-loop strength $\boldsymbol{\alpha}$. The curves in panel A separate the parameter space into areas with and without order conservation. At $\alpha=1$, full self-loop-strength, the curves seem to approach infinity. This is verified in panel $\mathrm{B}$, the dependent variable is transformed to $1-\tanh (b)$. In this depiction all curves approach zero. While panel A displays several curves for different values of $\varepsilon$, the curves are collapsed onto a single curve in panel $\mathrm{C}$, since $b$ and $\varepsilon$ occur together and are therefore considered as one parameter. The area below the curves in panel A corresponds to order conservation, in panel $\mathrm{B}$ and $\mathrm{C}$ above the curves, respectively.

to oscillator 1 for any oscillator is obtained through:

$$
\phi_{k}^{r}=-\sum_{k=1}^{i-1} \Delta_{k, k+1}^{r}=-\sum_{k=1}^{i-1} \lambda_{i} \Delta_{k, k+1}
$$

The new leading oscillator $\phi_{1}^{\text {new }}$ is the most phase advanced: $\phi_{1}^{\text {new }}=\max \left\{\phi_{1}^{r}, \phi_{2}^{r} \ldots\right\}$. Oscillator $\phi_{2}^{\text {new }}$ is obtained as the most phase advanced from the remaining oscillators: $\phi_{2}^{\text {new }}=\max \left\{\phi_{1}^{r}, \phi_{2}^{r} \ldots\right\} \backslash \phi_{1}^{\text {new }}$, and so forth. The new phase difference vector is obtained through $\Delta_{i, i+1}^{\text {new }}=\phi_{i}^{\text {new }}-\phi_{i+1}^{\text {new }}$.

\subsubsection{The Transition to Order Conservation with Self-Loops}

Order conservation exists if no interval $\Delta_{i, i+1}$ changes its sign across the block of spike receptions, therefore if $\lambda_{i}>0$ for all $i$. Since $\lambda(i)$ is monotonically decreasing, order conservation exists, if $\lambda_{N-1}>0$.

$$
\begin{aligned}
& \lambda_{N-1}=\left(e^{\varepsilon b}-e^{\alpha \varepsilon b}\right)+e^{\varepsilon b^{N-1}} e^{\alpha \varepsilon b}>0 \\
& \Longleftrightarrow e^{\varepsilon b(1-\alpha)}+e^{\varepsilon b^{N-1}}>1
\end{aligned}
$$

This condition for order conservation is identical to the condition derived in the paper of Hinrich et al. who only considered the case without self-loops $(\alpha=0)$.

Figure 4.8 shows areas of order conservation and overtaking within the parameter space for a system of $N=5$. With full self-loop strength $(\alpha=1)$ there is order conservation for all parameters. The transition to order conservation is continuous in the coupling strength parameter $\varepsilon$. 

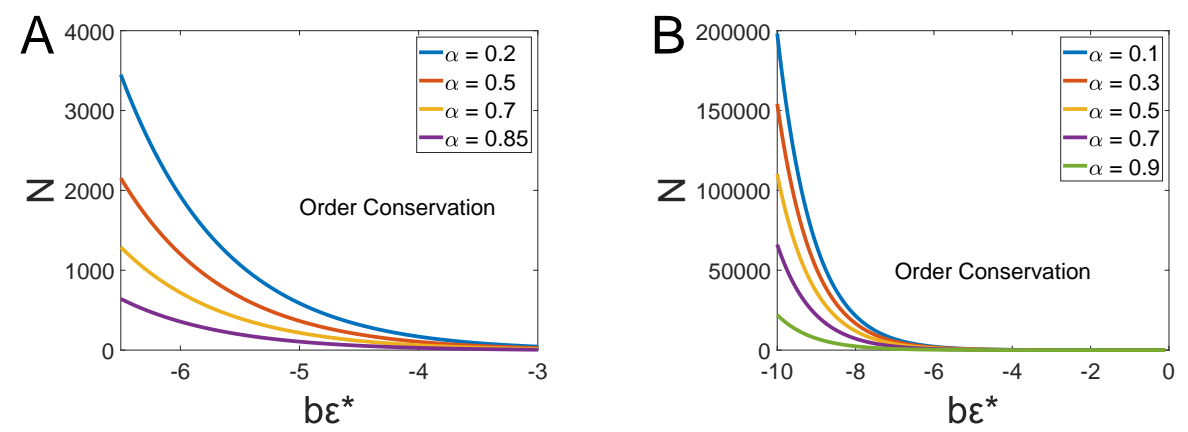

Figure 4.9: Scaled systems with $\boldsymbol{N}>\boldsymbol{N}_{\text {crit }}$ show order conservation. Each curve shows the critical value $N_{\text {crit }}$ in parameter space. Panel A shows $N_{\text {crit }}$ obtained by numerically solving the implicit condition for order conservation. Panel B shows the analytically obtained result for order conservation for large systems.

Order conservation depending on the system size. The zero crossing of $\lambda(i)$ and with it the bending point $i_{\text {bend }}$ are independent of the system size $N$ (see equation 4.8). Order conservation occurs in a system, if all $\lambda_{i}>0$. Therefore, by increasing the system size $N-1$ will surpass the zero crossing $i_{0}$ and since all $\lambda_{i}$ with $i>i_{0}$ are negative $\left(\lambda_{i>i_{0}}<0\right)$ order conservation is lost.

So far we have considered fixed coupling between two oscillators, the coupling strength between nodes $\varepsilon$ is a constant, independent of the network size. Since each node receives input from all other nodes of the network, the total input to each node from the network increases linearly with network size. In this paragraph only we will consider scaled coupling: $\varepsilon=\frac{\varepsilon *}{N}$. The coupling between two nodes $\varepsilon$ decreases with network size, such that the total input to a node from the rest of the network stays constant if the network size $N$ increases. Note that we keep the relative self-loop strength constant independent of network size: $\alpha=\frac{\sigma}{\varepsilon}$.

Analog to equation 4.9 the condition for order conservation is:

$$
e^{\frac{\varepsilon^{*} b}{N}(1-\alpha)}+e^{\frac{\varepsilon^{*} b}{N}(N-1)}>1
$$

Increasing the system size beyond a critical size will ensure order conservation. In figure 4.9 each line shows the critical system size $N_{\text {crit }}$ separating an area of order conservation from an area with breaking of order conservation in parameter space. For large systems the equation 4.10 can be solved analytically:

$$
\begin{gathered}
\lim _{n \rightarrow \infty} \frac{N-1}{N}=1 \\
e^{\frac{\varepsilon^{*} b}{N}(1-\alpha)}+e^{b \varepsilon *}>1 \\
N>N_{\text {crit }}=\frac{b \varepsilon^{*}(1-\alpha)}{\ln \left(1-e^{b \varepsilon^{*}}\right)}
\end{gathered}
$$

Figure 4.9, panel B displays the explicit solution for large systems. 


\subsubsection{Reordering Patterns within One Cycle}

The number of theoretically possible reorderings. The system size $N$ and the index of the bending point $k$ determine the set of possible reorderings given an arbitrary set of initial conditions (initial phase differences $\vec{\Delta}$ ). The oscillators with indices smaller than $k$ keep their ordering, while the oscillators with indices larger than $k$ fully flip their ordering, such that they are ordered in descending order to each other. These oscillators with indices larger than $k$ can end up in any position between the oscillators with indices smaller than $k$. E.g. In the system of size $N=7$ with a bending point $k=4$ any reordering is possible with the indices 1,2 and 3 in ascending order, 5,6 and 7 in descending order and 4 at the end or bottom of the reordering. Examples for possible permutations are: [1762354] or [7126354]. The size of the set of possible reorderings is given by:

$$
\frac{(N-1) !}{(k-1) !([N-1]-[k-1]) !}=\left(\begin{array}{c}
N-1 \\
k-1
\end{array}\right)
$$

Which of these permutations occurs, depends on the initial phase difference vector $\vec{\Delta}$ and on $\vec{\lambda}$, which biases the distribution of permutations, since stretching some intervals more than others makes some permutations more likely than others to occur given arbitrary initial phases. For $k=1$ the oscillators exactly change their order, there is no mixing, and for $k=N-1$ the order is fully conserved. The number of theoretically possible reorderings is maximal if $k$ is approximately half the system size. Since $k$ is independent of $N$, increasing the system size $N$ while keeping all other parameters constant will eventually surpass the point of optimal mixing. In large systems with $k$ small relative to $N$ the amount of theoretically possible reorderings is small compared to the theoretically possible reorderings in a situation with optimal mixing with $k$ approximately half the system size.

All reorderings occur in the system. In the previous section we derived the number of theoretically possible reorderings given a certain parameter set. But not all theoretically possible reordering occur equally often, since the vector $\vec{\lambda}$ renders some reorderings to occur more often than others given uniformly distributed initial phase differences. Here we study how the distribution of realized permutations depends on the relative self-loop strength $\alpha$ for otherwise default parameters, including $N=7$. In Figure 4.10 each panel shows a histogram for a different $\alpha$-value.

The theoretically possible reorderings emerge with increasing $\alpha$ from 0 to 0.99 as predicted by the binomial coefficient: Within panel A-E the bending point is $k=2$ and 6 different permutations occur. The bending point increases to $k=3$ (15 permutations) in panel $\mathrm{F}$ and $\mathrm{G}, k=4$ (20 permutations) in panel $\mathrm{H}$ and $\mathrm{I}$, $k=5$ (15 permutations) in panel $\mathrm{J}, k=6$ (6 permutations) in panel $\mathrm{K}$ and $k=7$ (1 permutaton) in panel L. The observed distributions are far from uniform. E.g. in panel A the permutation [7654312] occurs far more often than any other. Due to the shape of $\lambda(i)$ and $k=2$ (see figure 4.4 the only interval not changing its order, $\Delta_{1,2}$ shrinks strongly, therefore the first oscillator is likely to remain neighboring to the second as observed. For intermediate values of $\alpha$ permutations are more evenly distributed and maximal mixing occurs. For $\alpha=0.95$, the permutation is heavily 

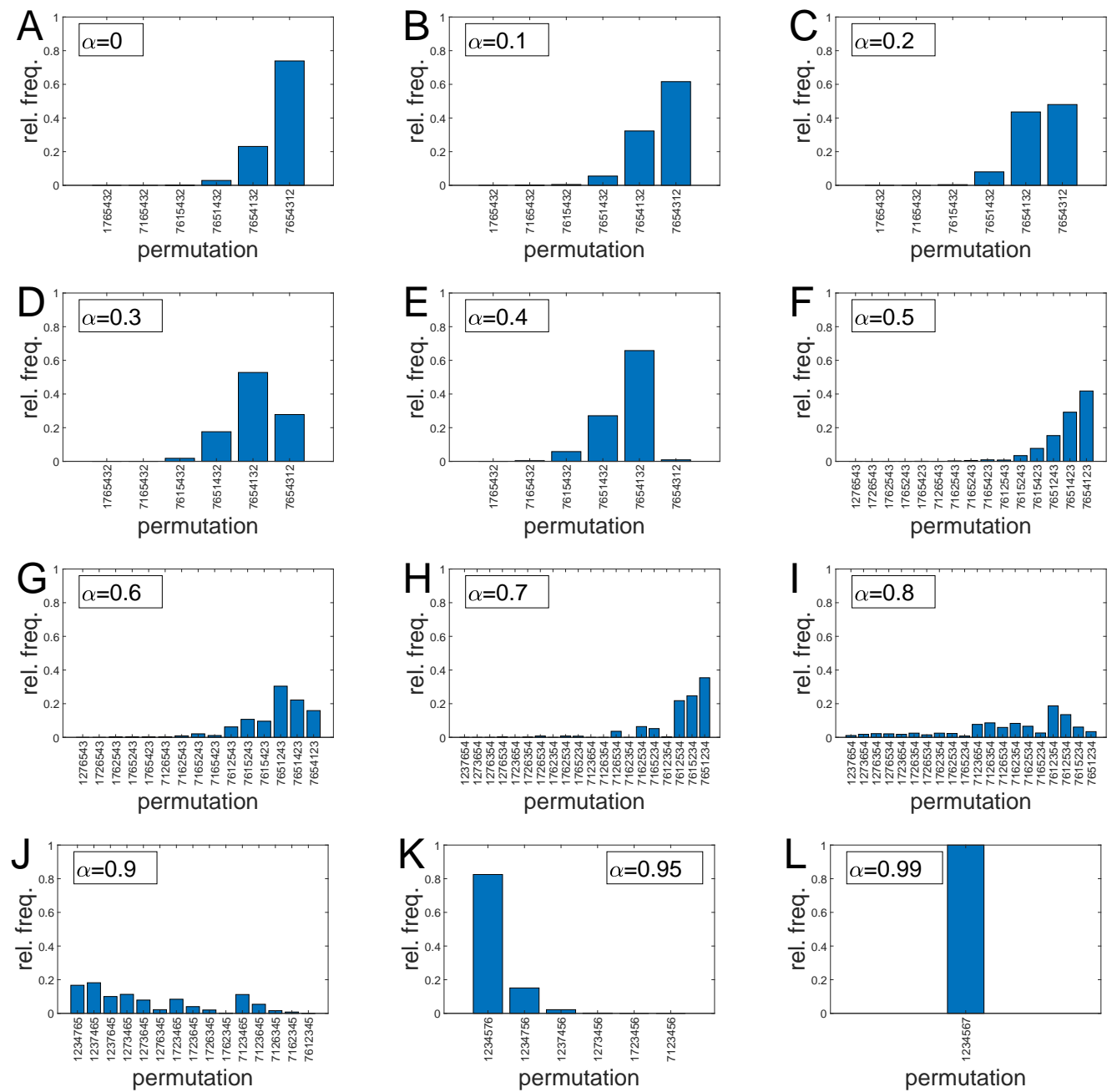

Figure 4.10: Normalized histograms of reorderings given random initial conditions. For each plot 1000 random initial conditions were generated by drawing initial phases uniformly between zero and one for each oscillator. They were then ordered and named according to their order. For each possible reordering the amount of occurence normalized by 1000 is displayed. The parameters used are: $N=7, \varepsilon=-0.2$ and $b=3 . \alpha$ is varied across panels. 

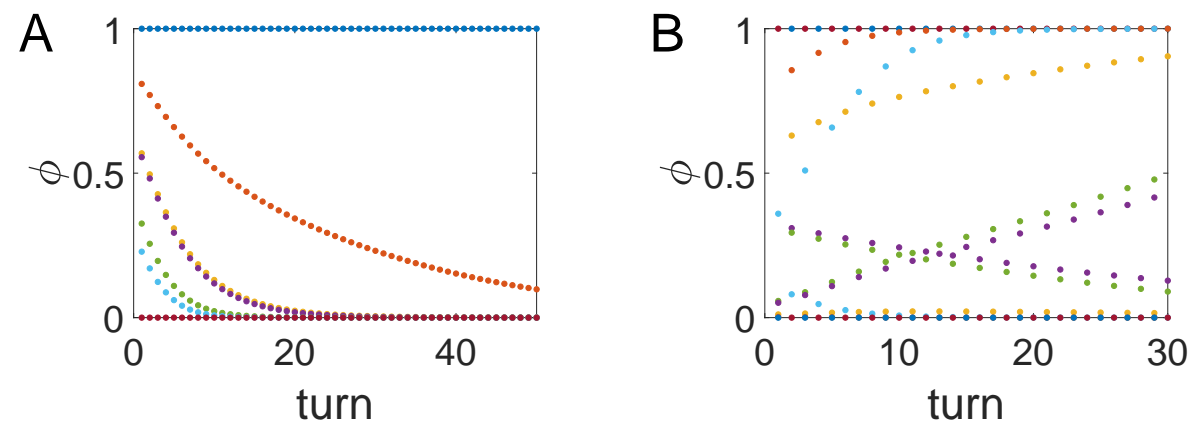

Figure 4.11: Trajectories of reorderings with simple patterns. Oscillator identity is marked by identical color throughout the simulation. Panel A shows 50 steps starting from equally spaced initial conditions, $b=1$, otherwise default parameters were used. Panel B shows 30 steps starting from equally spaced initial conditions, $b=5.6$, otherwise default parameters were used.

skewed towards [1234576], analog to panel A, the only interval changing its order shrinks a lot, therefore oscillator 6 and 7 are likely to remain neighbors. In the last panel we see order conservation. In this section the transition to order conservation by increasing self-loop strength was shown from a different angle.

\subsubsection{Reordering Patterns Across Cycles}

So far we have only considered reorderings across one block of spike receptions. Here we study reorderings across several steps, each step defined as a full spike reception event. The assumption of initially well separated spike sending and spike reception events is automatically ensured over several steps, since distances between oscillators always shrink, since $\left|\lambda_{i}\right| \leq 1$. Spike sending and reception events become increasingly separated.

Figures 4.11, 4.12, 4.13 and 4.14 show trajectories across several steps. Oscillator identity is marked by identical color throughout the simulation. In each step the total distance between the oscillators is renormalized to 1 to counteract the contraction due to $\left|\lambda_{i}\right|<1$. Since this has no effect on the relative interval sizes, the evolution of the orderings of the oscillators is not affected by the normalization.

Simple patterns of reordering. Figure 4.11 shows two trajectories with simple reordering patterns in a system of size $N=7$. In panel A all oscillators keep their ordering, since $k=1$. In panel B the oscillators fully reverse their ordering in each step due to $k=7$.

Chaotic mixing. In figure 4.12 the oscillators mix seemingly in a random fashion, despite the deterministic nature of the system, a property often found in chaotic systems. Furthermore, the mixing results from a folding mechanism, a common mechanism to produce chaos. However, the overall dynamics of the system (without the 

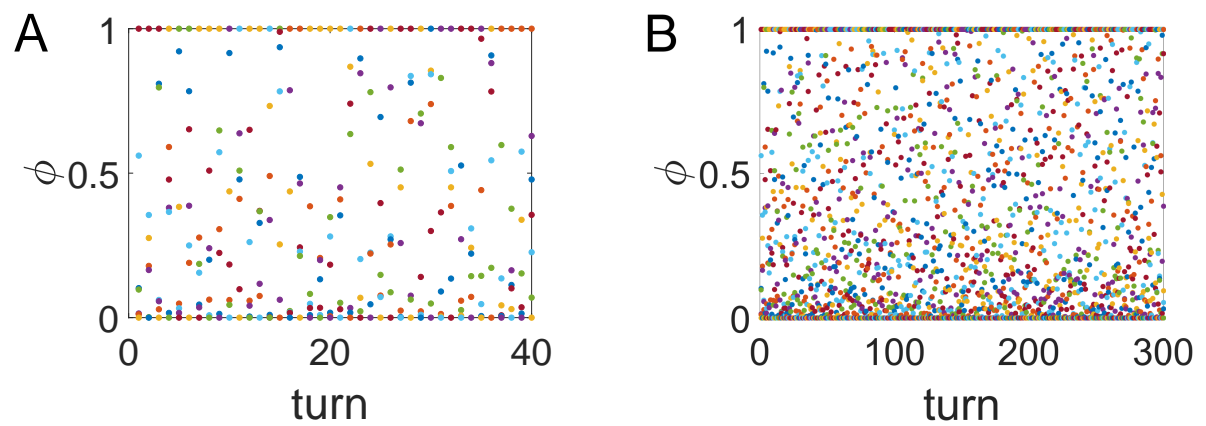

Figure 4.12: Trajectory with chaotic mixing. Panel A shows the first 40 steps, panel $\mathrm{B}$ the first 300 , of the same trajectory, $b=2.2$, otherwise default parameters were used. Initial conditions were chosen randomly.
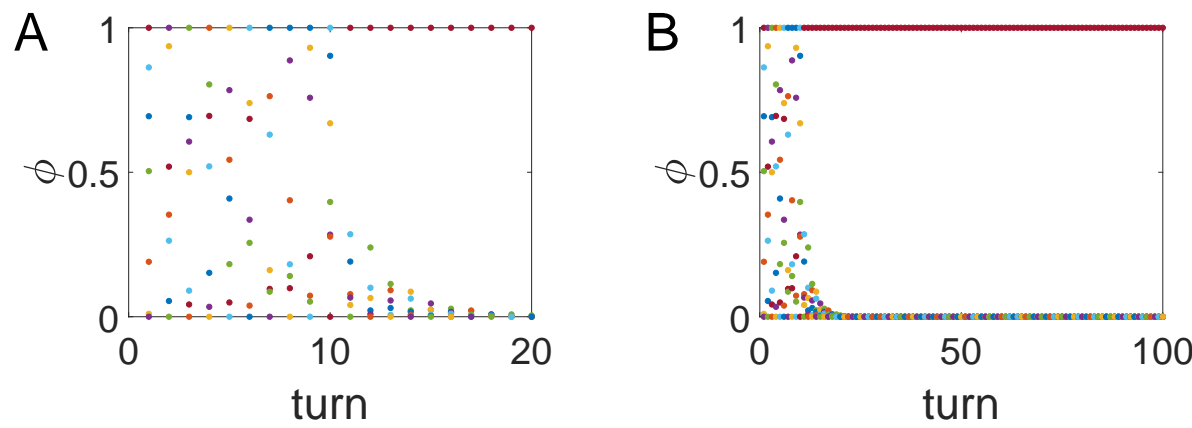

Figure 4.13: Trajectory showing separation of one oscillator. Panel A and B show the first 20 and 100 steps of the same trajectory, respectively. At step 11 the dark red oscillator takes the up most position and remains there to the end of the simulation, while the other oscillators continue to mix. $b=2$, otherwise default parameters were used.

renormalization) approach a fixed point, hence, the system does not have a chaotic attractor.

Separation of oscillators. Figure 4.13 shows a trajectory for $b=2$. The first 10 steps resemble the chaotic mixing observed in figure 4.12. The oscillators seem to randomly scatter over the space. But then at step 11 the dark red oscillator takes on position 1 and from then onward stays in the first position. The distance to the other oscillators, which continue mixing, seems to grow exponentially so that in the end all other oscillators are close to zero, while the dark red oscillator remains at 1. Even after 100 steps of simulation the situation has not changed.

In the next figure 4.14 we return to the same parameter set of figure 4.12 . Here we can see a similar situation as in figure 4.13, from step 7 onward the dark red oscillator occupies the first position, all the other oscillators are rather close to zero. But, in contrast to the trajectory in figure 4.13 discussed above, this separation is not permanent, after several steps the dark red oscillator leaves the first position and the system returns to the state of full mixing. It seems that in the trajectory 

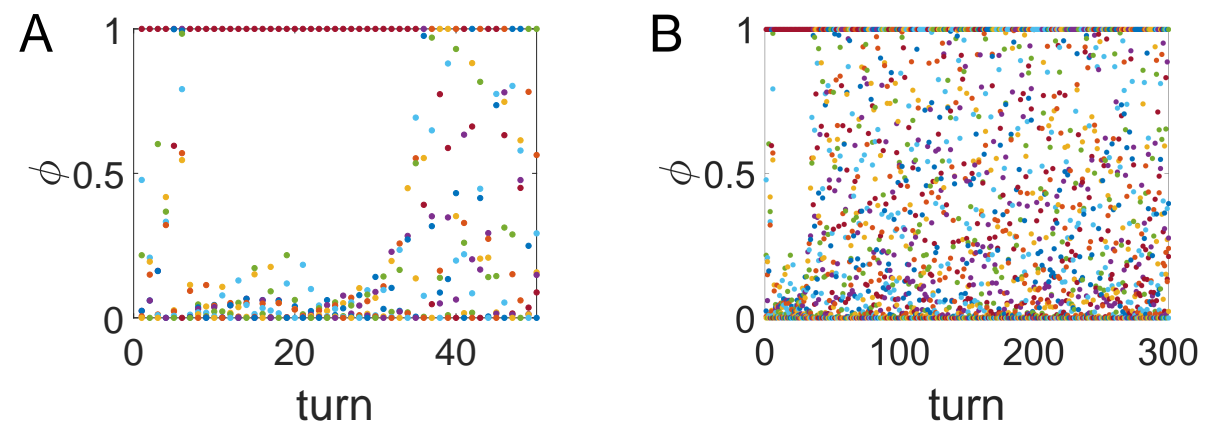

Figure 4.14: Recovery of an oscillator's separation. Panel A and B show the first 50 and 300 steps of the same trajectory. At step 7 the dark red oscillator separates from the others, however, at step 36 it rejoins the mixing procedure. Identical parameters as in figure 4.12 were used.
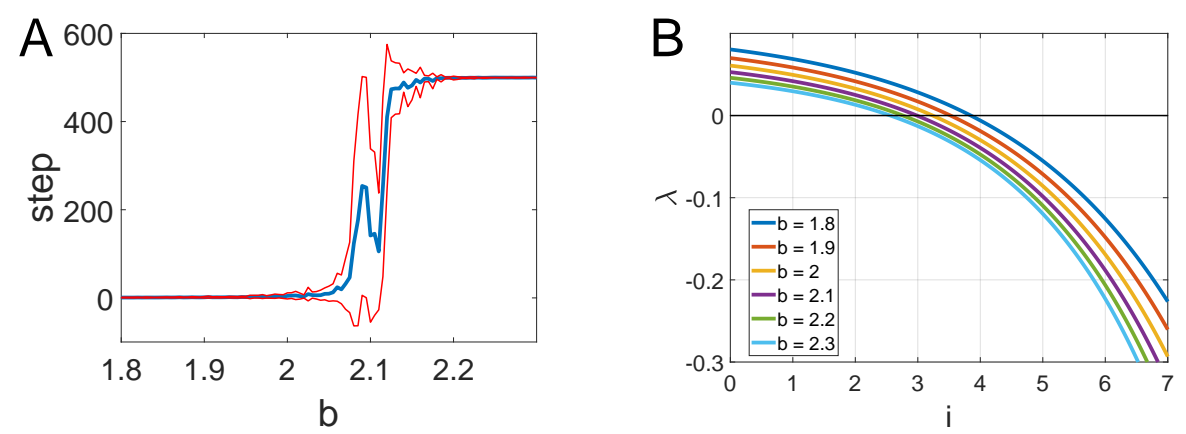

Figure 4.15: Average separation time depending on b. Panel A: For each value of $b 40$ trajectories with random initial conditions were simulated for 500 steps each. The separation step was defined as the step from which onward the upmost oscillator remained identical until the end of simulation. If no oscillator ever separated, time to separation is measured as 500. The blue line depicts the average over all trials. The two red lines depict average plus and minus one standard deviation. A transition around $b_{t}=2.1$ is observed from near immediate separation for $b<b_{t}$ and no separation within simulation time for $b>b_{t}$. Panel B shows corresponding $\lambda(i)$ for selected values of $b$. Otherwise default parameters were used. 
with full separation (figure 4.13 there is a mechanism that lets the one oscillator separate exponentially from the others and ensures that it stays separated, while this mechanism is not present in the trajectory in figure 4.14. However, we have not yet understood such a potential mechanism and can only speculate.

For $b=2.2$ we have seen an example of a trajectory that shows chaotic mixing to the end of simulation time (figure 4.12) as well as an example for full separation of one oscillator (figure 4.13). For $b=2$ we have seen an example of an oscillator at the brink of separation, but rejoining the mixing procedure.

In the figure 4.15 we estimated the average times until separation of one oscillator from the rest depending on the parameter $b$. Each data point was optained from 40 simulations with random initial conditions. Since each trajectory was simulated for 500 time steps the time until separation levels of at 500. However, a transition occurs around $b=2.1$. The ziczac shape in the center of the phase transition curve coincides with the change of the bending point from $k=4$ to $k=3$. The data indicate that increasing $b$ beyond $b=2.1$ leads to more separation events. Why this is remains an open question.

\subsection{Summary and Discussion}

Golubitsky et al. [25] showed that in a symmetrical all-to-all coupled network which can be described by ODEs invariant sets exist, polydiagonals defined by two or more units synchronized. These separate the full phase space into disjoined volumes. Each volume corresponds to a specific ordering of the network units. Due to continuity of trajectories and uniqueness of flow the flow invariant subspaces serve as barriers for the flow. Trajectories are confined to their initial volume and the units show order conservation.

Kielblock et al. [38] demonstrated that the same reasoning does not apply to symmetric all-to-all coupled networks of pulse coupled oscillators. They provide examples that such systems do not show order conservation, even if the pulse-coupling has no delay and does not induce any non-smoothness.

Kielblock et al. as well as Golubitsky et al. consider all-to-all coupled networks without self-coupling. In this chapter we build on the work of Kielblock et al. and introduced self-coupling gradually into pulse-coupled systems. We observed in two example systems, in a smooth pulse-coupled system and in a system coupled via delayed $\delta$-pulses, that self-coupling restores order conservation.

The system with delayed $\delta$-pulse-coupling allowed for analytic treatment. We assumed, that spike receptions occur blocked and derived a return map, that provides the phase differences after reception of all spikes depending on the phase differences before. We fully described the mechanism behind the reordering process including a folding procedure, derived conditions for order conservation and showed that it is indeed enforced by full self-coupling. The reordering patters show rich dynamics, including simple patterns as well as chaos-like reordering. We observed that single oscillators may separate themselves from the reordering process, a phenomenon not yet fully understood. The average time to separation depending on parameters was numerically estimated. 


\section{Theoretical Considerations on Symmetrical Pulse-Coupled Systems}

How is it possible that the reasoning applied by Golubitsky et al. does not apply to pulse-coupled systems, even if pulses are used, that induce no non-smoothness when received?

To fully characterize the current state of a pulse-coupled system it is not sufficient to provide the values of the dynamical variables. Pulses sent in the past may still influence the future of the system. Therefore additional knowledge is required of the sending times of all pulses, that may still influence the system's future. Kielblock at al. hypothesized that trajectories can avoid the invariant manifolds in the space spanned by dynamical variables and can move around them in the additional dimensions, that arise through the pulses. In the lower dimensional space spanned by the dynamical variables it appears as though the trajectories can cross the invariant manifolds.

In the following paragraphs we would like to expand a bit on these thoughts.

\section{Invariant Sets as Barriers in a Symmetrical ODE System}

Our starting point is the system considered by Golubitsky: In a symmetrical all-toall coupled network described by ODEs, two units generally receive identical input from all the other units in the network besides themselves, because any other unit is connected to both of them with equal strenght. Each unit in the network has identical intrinsic dynamics and processes received inputs in the same way. When they are synchronized the input each of the two units receives from the other is identical. Therefore they have identical dynamics and stay synchronized. Hence, the states in phase space where two or more units are synchronized form flow invariant subsets of space. They are polydiagonals, that separate the whole phase space into volumes, each corresponding to a specific ordering of the units. In an ODE system the existence and uniqueness theorem holds. Because trajectories cannot merge or split, they cannot cross the flow invariant subspaces and are confined to their initial volume of space, which results in order conservation.

\section{Invariant Sets and Additional Dimensions in a Symmetrical Pulse-Coupled System}

If pulse-coupling is introduced into such a system, then the input from any of the other units of the network to the two units is still identical. The intrinsic dynamics of each unit in the network are identical and inputs are processed the same way by each unit. If the two units have been synchronized in the past, they receive identical input from each other, because their pulse sending times and pulse intensities are synchronized. Therefore they have identical dynamics and will stay synchronized.

However the following scenario is also possible: Two oscillators can have the same phase, again they receive identical input from the rest of the network and have identical intrinsic dynamics. But if they were not synchronized in the past and sent pulses at different times, these differences from the past translate into different pulse intensities received at the current time point when the two units are momentarily synchronized. So they do not receive the same input from one another at this point in time, despite having the same value, and they split. The qualitative difference in an ODE system with instantaneous state dependent coupling and a pulse coupled system 
arises, because in an ODE system differences in the past do not have any effect onto the current evolution of the system, while in a pulse-coupled system, the information on value differences in the past remains in the system, the value differences are represented through non-synchronized pulses, which still effect the current evolution of the system.

How to make sense of these observations with regards to the phase space of pulsecoupled systems?

A subspace, that is defined by one constraint, has one dimension less than the full space. If this subspace is a hyperplane, it separates the full space into disjoined volumes. The subspace defined by two units synchronized is defined by imposing one constraint on the full space, it is a hyperplane and therefore separates the full space into disjoined volumes (including the dimensions from all pulses sent in the past, that may still influence the system's future). However, since units on this hyperplane do not generally receive identical input, the hyperplane is not flow invariant.

The subspace defined by two units being synchronized and having been synchronized in the past sufficiently long, so that all pulses sent by these two units, that may still influence the system's future, are also synchronized, is flow invariant. However, this subspace is defined by more than one constraint (if the set of pulses sent in the past by these two units, that still influence the future of the system is not empty). Therefore it does not separate the full space into disjoined volumes. The subspace does not act as a barrier between the volumes corresponding to one ordering of the two units or the other, because it is too low dimensional. Therefore the reasoning of Golubitsky et al. cannot be extended to pulse-coupled systems and they can overtake each other.

Also we would like to add a final remark on the question, whether trajectories in a pulse-coupled system can join or split or cross each other: A pulse-coupled system has the peculiar property of dynamically changing the dimensionality of the space that it is evolving in. In such a space, the evolution of two close by trajectories can be such, that one of the two trajectories may add another dimension to the space through pulse generation, while the other does not. Now the two trajectories are not part of the same space anymore. Is it meaningful to ask, whether two trajectories can merge or split, if they are not part of the same space? This surely involves conceptual problems. What we are usually interested in is the subspace of the pulse-coupled system spanned by the dynamic variables themselves (and not by the additional dimensions deriving from the pulses), hence we are interested in a lower dimensional projection of the full space. This space remains intact throughout the full evolution of the system. And therefore comparisons between trajectories in this lower dimensional projection are meaningful and possible. However, this projected space is not the space that many of the central theorems of dynamical systems' theory are concerned with, e.g. the continuity of trajectories and uniqueness of flow theorem does not apply here.

Creating new dimensions and thereby dynamically changing the phase space they live in is a peculiar property of pulse-coupled systems and certainly needs more investigation to be properly conceptualized, understood and treated. 


\section{Theoretical Considerations on Introducing Self-Loops}

We demonstrated numerically that introducing self-loops into the system with smooth coupling studied by Kielblock et al. restores order conservation. Here we will discuss why:

In a system with self-loops all network units always receive identical input, since they receive input from every node of the network including themselves. All units also have identical intrinsic dynamics and process input the same way. Hence the subspace with two units synchronized is flow invariant.

For any network system it is possible to define a corresponding system with replacing the interactions between the units by external input provided to each unit separately. This turns the original network into a virtual network, however the dynamics of each unit stay the same.

This new system representation is described by ODEs, does not entail pulse-coupling and has an explicit time dependence. Because the pulses as chosen by Kielblock et al. were constructed in a way that ensure smoothness, the new system is also smooth. Through substituting time with a dynamical variable, that evolves with speed 1 , it is possible to define a system with identical dynamics, that is autonomous, while having an additional dimension (this substitution is explicitly presented in chapter 6). Finally in this new system the existence and uniqueness theorem holds, because it is a smooth autonomous ODE system.

In this smooth autonomous ODE system the subspace of two units synchronized is still flow invariant, since the dynamics of each unit were not changed through the system transformations. Further, the subspace with two units synchronized imposes one restriction on the full space of all $N+1$ dimensions. Therefore it fully separates the full space into two volumes, each corresponding to a specific ordering of the two units. Because the existence and uniqueness theorem holds a trajectory starting in one volume cannot cross the flow invariant subspace and therefore order is conserved. This is also true for the original system, since none of the system transformations changes the dynamics of the units themselves.

The same reasoning does not apply to introducing $\delta$-pulses. While the subspace of two units synchronized is still flow invariant and separates the full $N+1$ dimensional transformed space, the existence and uniqueness theorem does not hold, the system is not smooth, trajectories can jump and therefore cross the flow invariant subspace. Here we conjecture that it is possible to find a symmetrical $\delta$-pulse coupled network system with self-loops that does not show order conservation.

However, the $\delta$-pulse coupled system presented in this chapter does show order conservation if self-loops are introduced. To ensure, that a system with $\delta$-pulses shows order conservation, we considered a transfer function, that is strictly monotonically increasing $\left(H_{\varepsilon}(\phi)=e^{b \varepsilon}>0\right)$. The transfer function provides the phase after $\delta$ pulse reception given the phase before the pulse is received. Since this function is monotonically increasing, the spike reception is a neutral operation with regards to the ordering of the oscillators.

One caveat: we multiply the $\delta$-pulse with the interaction function $Z(\phi)=H_{\varepsilon}(\phi)-\phi$. We interpret the $\delta$-pulse as a discrete jump with the size provided by the interaction function. Very different dynamics can arise, if the $\delta$-pulse is considered to be the limiting function of a smooth kernel, e.g. a bump function. The resulting system 
can show completely different dynamics (e.g. see reference [14 for a more detailed analysis).

\section{Context of the Thesis}

In this thesis we want to study the richness of dynamics that can arise in very simple systems when introducing dynamical features commonly used in theoretical neuroscience. In this chapter we build our research on previous work by Kielblock et al., who demonstrated that introducing pulse-coupling into a symmetrical oscillator network enables qualitatively different behavior not possible in an otherwise identical ODE system: overtaking of oscillators. We expanded their work by introducing selfloops and studied the mechanisms behind the reordering process. We found rich dynamics including chaos-like mixing and patterned reordering depending on the system's parameters. 



\section{Chapter 5}

\section{Chaos in Two Pulse-Coupled Phase Oscillators}

In this thesis we study the implications that arise if dynamical features commonly used in theoretical neuroscience are introduced into simple systems. In the last chapter we studied the effect of pulse-coupling with a special focus on delayed $\delta$-pulses. In this chapter we will continue studying delayed $\delta$-pulse-coupling.

We consider a network of only two phase oscillators coupled via exchanging delayed $\delta$-pulses. We find that the network can show chaotic behavior. This is not possible in a two phase oscillator ODE network (which has instantaneous state dependent coupling). Hence we provide another example for a system with delayed $\delta$-pulsecoupling that behaves fundamentally different to the corresponding ODE system from a dynamical systems' perspective. Far richer dynamics are possible.

\subsection{Introduction}

\section{Chaos in Dynamical Systems}

Since the discovery of chaos in 1963 by Lorenz [49] chaos research has become a focus of interest in the field of dynamical system theory. With the help of chaos theory insights could be gained in phenomena such as turbulence and weather dynamics. Also applications of chaos have been found such as encrypted communication [96. Others study it for its intrinsic beauty [80. The fascination of chaos stems from the seeming paradox of apparent random behavior in a fully deterministic system. In a system of smooth ODEs chaos can only occur if the system is at least three dimensional. This follows from topological constraints regarding trajectories in a two dimensional phase plane (Poincaré-Bendixson-theorem). Examples for such three dimensional chaotic attractors include the Lorenz attractor [49] and the Rössler attractor [72].

\section{Examples for Chaos in Low-Dimensional Systems}

While chaos is a common to high-dimensional nonlinear systems, it occurs much less in low dimensional systems. The Poincaré-Bendixson-theorem considers possible phase space topologies in the plane. As direct consequence of this theorem it follows that chaos in a system described by smooth ODEs requires at least three dimensions. Hence, continuous-time chaotic dynamical systems with two or one dynamical variables need to entail some special feature to get around this restriction. There are some example systems in the literature: 
Dixon et al. [18 study the magnetization of a neutron star from a theoretical perspective. They arrive at a simple system of two nonlinear ODEs. Simulation shows chaotic behavior and a positive Lyapunov exponent. All trajectories seemingly pass through a single point, which they leave in an apparent random direction. However, this finding is an artifact of numerical instability. The singularity arises due to nondifferentiability of the two equations at this point, where all trajectories merge into a single one.

Another example is provided by Nakano and Saito [61]. They present a hybrid system of two differential equation which produce outwards spiraling trajectories. Each time a threshold is reached trajectories are mapped to another coordinate in phase space. The phase space portrait resembles a two dimensional projection of the Rössler attractor.

Nosov et al. 65] present a commutable pendulum, another example of a chaotic system with only two dynamical variables. Each time the system passes from one region in phase space to another the dynamical equations describing the system switch, realized by changing a parameter value depending on the exact switching point.

$\mathrm{Xu}$ [95] presents a kicked harmonic oscillator, a two dimensional system that receives $\delta$-pulses at regular intervals, weighted with a position-dependent sinusoidal function, which shows chaotic behavior.

\section{The Role of Chaos in Neuroscience}

The question whether or not neural dynamics are chaotic is a long-standing issue in theoretical neuroscience. (e.g. [28]).

Some researchers believe that chaotic neural dynamics may play a functional role in the brain, e.g. Aronov et al. [6] observe a specific brain area, which is responsible for generating random vocalization in young song birds, comparable to human babbling. The produced variability is likely to be necessary for learning new vocalization patterns. The authors hypothesize that the neural dynamics within this brain area are chaotic. Another very different interest in whether neural dynamics are chaotic stems from the long lasting debate in theoretical neuroscience concerning the question on how information is encoded in neural activity. Is the information carried by the firing rates alone or are precise spike timings important? Monteforte and Wolf ([59] argue that if the spiking dynamics are chaotic, small perturbations, e.g. produced by ion channel noise, will exponentially grow. If this growth is fast enough information carried by the spike timings is lost quickly, which makes spike timings unlikely to carry the fundamental code underlying neural computations.

Research on chaotic neural dynamics often involves large neural networks (e.g. [12, 36, [51, [59, 76]).

Many large neural networks produce irregular spiking dynamics which arise from a balanced state network architecture [88, 89, in particular, for $\delta$-pulse-coupling. (e.g. [36, 51, 59]. However, Jahnke et al. [36] as well as Manz et al. [51] demonstrated that the irregular dynamics arising in large balanced state networks may be chaotic or not, depending on the dynamical properties of the network units, i.e. whether the coupling is excitatory or inhibitory [36] or whether the neuron models used are leaky integrators (with positive dissipation) or anti-leaky integrators (with negative dissipation) [51. The question arises, whether large networks are necessary for chaos 
to arise in neural systems. Maybe certain neural features themselves are sufficient to produce chaos already in simple, low dimensional dynamical systems. Accordingly, in this chapter we present a simple system with delayed $\delta$-pulse-coupling, a common neural feature. The system consists of only two interacting phase oscillators and shows chaotic behavior. Delayed $\delta$-pulse-coupling seems sufficient to produce rich dynamics such as chaos in a very simple system.

This chapter is structured as follows: In the second section we define the system. In the third section we demonstrate that this system shows the typical signature found in chaotic system. In the fourth section we show that the observed behavior is related to chaos arising in unimodal maps. Finally, in the last two section we discuss and summarize the results.

\subsection{System Definition}

We consider a symmetrical system of two $\delta$-pulse-coupled phase oscillators. The system is described by the following two differential equations:

$$
\begin{aligned}
& \dot{\phi}_{1}=\omega+Z\left(\phi_{1}\right) \sum_{m} \varepsilon K\left(t-t_{2}^{m}\right), \\
& \dot{\phi}_{2}=\omega+Z\left(\phi_{2}\right) \sum_{n} \varepsilon K\left(t-t_{1}^{n}\right) .
\end{aligned}
$$

The phase speed $\omega=1$ describes the intrinsic dynamics of each oscillator. If the phase reaches the firing threshold $\phi=1$ the oscillator sends a $\delta$-pulse, we will call spike, and resets:

$$
\phi_{i}\left(t_{i}^{m-}\right)=1 \rightarrow \phi_{i}\left(t_{i}^{m+}\right)=0 .
$$

$t_{i}^{m}$ denotes the $m$-th time oscillator $i \in 1,2$ sent a spike, $t_{i}^{m-}$ denotes the time just before reset, $t_{i}^{m+}$ just after reset. The $\delta$-pulse is received by the other oscillator after a fixed time delay $\tau>0$, which is expressed by the coupling kernel $K$ :

$$
K\left(t-t_{i}^{m}\right)=\delta\left(t-t_{i}^{m}-\tau\right) .
$$

Each incoming $\delta$-pulse is weighted by the coupling constant $\varepsilon>0$. Further, it is weighted by the phase response curve $Z(\phi)$. The susceptibility to an incoming $\delta$ pulse varies throughout the cycle of each oscillator:

$$
Z(\phi)=\left(\phi-\phi_{0}\right)(\phi-1)
$$

with $\phi_{0}=0.2$. This quadratic function has two zero crossings: $\phi=0.2$ and $\phi=1$. Figure 5.1 displays the phase response curve $Z(\phi)$ and the coupling kernel $K\left(t-t_{j}^{m}\right)$.

Default parameter set. If not noted otherwise the following parameters are used throughout the chapter as default: $\varepsilon=2.7$ and $\tau=0.66$. The default initial phases of both oscillators are $\phi_{2}=0.58$ and $\phi_{1}=0$ (right after reset). Hence, there is exactly one spike sending time relevant for the system's future, which is $t_{1}^{1}=0$ ). 

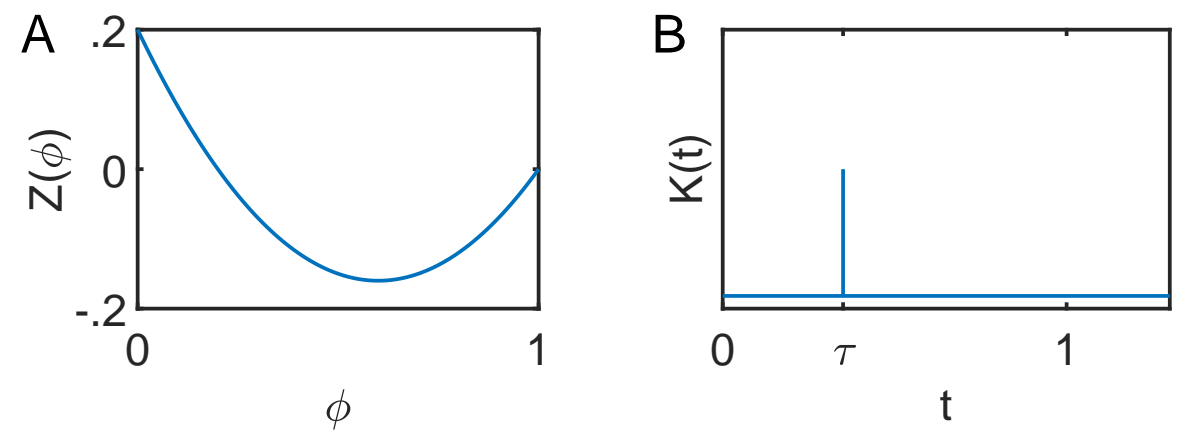

Figure 5.1: Phase response curve $Z(\phi)$ and the coupling kernel $K(t)$ consisting of a delayed $\delta$-pulse.
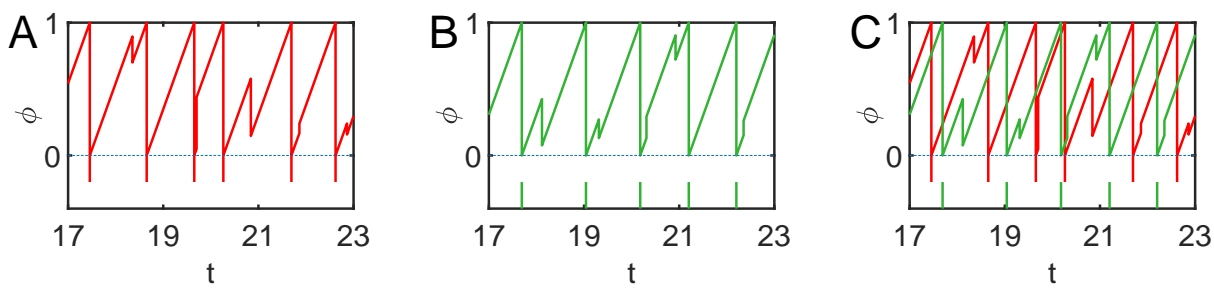

Figure 5.2: Phase trajectories of both oscillators. Depicted are the phases over time. Panel A shows the first oscillator, panel B the second during the same time window. Panel $\mathrm{C}$ shows a superposition of both trajectories. While the underlying dynamics are deterministic the trajectories do not follow a pattern but appear to behave randomly.

\subsection{Signature of Chaos}

In this section we will demonstrate that the typical signature found in chaotic systems is found in this system as well.

\section{Trajectories}

Irregular trajectories. Trajectories of chaotic systems look irregular, they do not fall into a repetitive pattern but seem to continue in a random fashion despite the underlying deterministic dynamics. The phase trajectories of the two oscillators show this kind of irregular behavior. While the phases between spiking events proceed with constant speed $\dot{\phi}=1$, receiving spikes introduces jumps of varying sizes into the trajectories. These varying jumps, mediated through the phase response curve, create the overall irregular trajectories (see figure 5.2).

Nearby trajectories diverge. A chaotic system is sensitive to small perturbations. If a trajectory is perturbed only slightly it will separate exponentially fast from the unperturbed trajectory. The perturbation leads to very different long term behavior of both trajectories. In figure 5.3 we show unperturbed and perturbed phase trajectories of both oscillators. The two trajectories separate further at each spike reception event 

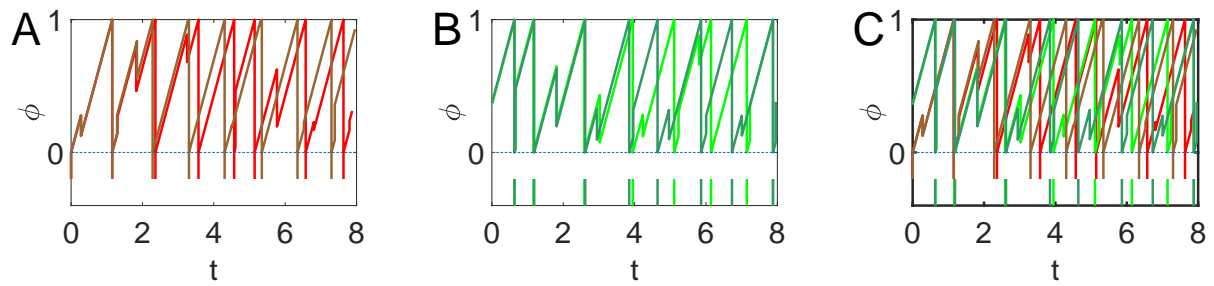

Figure 5.3: Unperturbed and perturbed phase trajectories of both oscillators closed to the chaotic attractor. Default parameters and initial conditions were used. To ensure closeness to the presumed chaotic attractor an initial transient of 1500 resets of the first oscillator was discarded. Then the phases of both oscillators were perturbed by adding 0.01. $t=0$ denotes the time of perturbation. Panel A and B show unperturbed and perturbed trajectories of oscillator 1 and 2, respectively. Panel $\mathrm{C}$ shows all trajectories superposed. The perturbed trajectories are shown in darker colors. At each spike reception event the trajectories separate further until their behavior differs completely.

until they eventually show unrelated behavior.

\section{Orbit Diagram}

An orbit diagram has become an iconic depiction of chaotic behavior. It shows the system's behavior across a range of parameter values. In a one-dimensional timediscrete system, such as the logistic map [54], the diagram is obtained by plotting the values of the dynamic variable of several consecutive iteration steps on the attractor against each parameter value. In the book 'Nonlinear Dynamics and Chaos' by Strogatz, p. 364 [82, the orbit diagram for the logistic map is depicted. If the trajectory for a given parameter value is at a fixed point only this point appears in the diagram, if the trajectory is a cycle of length two, the two points are plotted and so forth. If the trajectory is aperiodic, a cloud of points will appear in the diagram. Therefore, the diagram allows for a quick overview of periodic and aperiodic behavior within the parameter range of interest. In a time continuous system, such as the Rössler system [72], the consecutive points plotted against each parameter value, can be the values of local maxima of the trajectory in one dimension. A depiction can be found on page 386 in the same book. If the maxima appear always at the same height, only a single point is plotted for the given parameter value in the diagram. If the maxima alternate between two heights, then two points are plotted and so forth. Again the diagram allows for a quick overview on periodic and aperiodic windows of behavior within the parameter range of interest. Both orbit diagrams mentioned above, even though obtained from very different systems and through different methods, exhibit remarkably similar structures.

To obtain figure 5.4 we vary the delay $\tau$. For each parameter value we do a Poincaré section of the system each time the first oscillator resets and we plot the phase of the second oscillator $\phi_{2}$. The orbit diagram we obtain shows windows of periodic behavior interspersed with parameter regions with apparently no periodic behavior and exhibits 

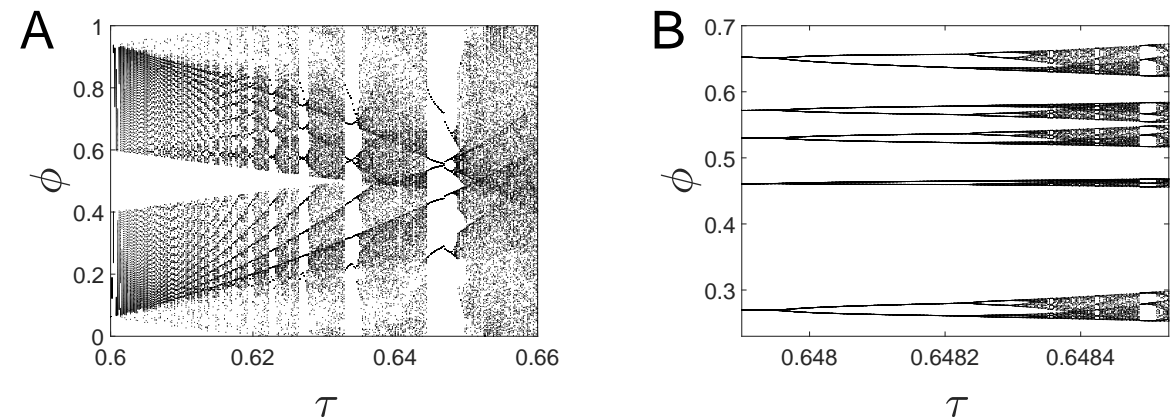

Figure 5.4: Orbit diagram across varying $\tau$. For each parameter value $\tau$ the phase of the second oscillator $\phi_{2}$ is plotted at 300 consecutive reset times of the first oscillator. An initial transient of 200 resets was discarded to ensure closeness to the presumed chaotic attractor. The second panel shows an expanded region from the first. Self-similarity as well as period doubling can be observed. Besides $\tau$, default parameters and initial conditions were used.

some similarity to the above mentioned examples. An enlarged region reveals a period doubling cascade to chaos, starting with a period-5 orbit. Within the finer structure of the enlarged region further windows of periodic behavior interspersed into regions with chaotic behavior appear, pointing towards a possible fractal structure, also common to chaotic systems.

\section{Maximal Lyapunov Exponent}

Chaos is characterized by a positive maximal Lyapunov exponent. We adapted the method to numerically estimate the Lyapunov exponent from Jahnke et al. [36]. The state of the system at any point in time is fully described by the two phases of the oscillators $\phi_{1}$ and $\phi_{2}$ and all spikes that were sent within the system's past of length $\tau$, since these spikes will be received in the system's future. Therefore each generated spike effectively adds an additional dimension to the system. Once the spike is received, the additional dimension corresponding to that spike can be discarded again, since it has no further effect on the system. Similar to Jahnke et al. [36] for each spike time $t_{i}^{m}$ we introduce a further dynamic variable $\sigma_{i}^{m}(t)=t-t_{i}^{m} \in[0, \tau]$ measuring the time passed since this spike. At time $t_{i}^{m}, \sigma_{i}^{m}\left(t_{i}^{m}\right)=0 . \sigma_{i}^{m}$ increases with constant speed $\left(\dot{\sigma}_{i}^{m}=1\right)$ until the spike sending time is reached $\left(\sigma_{i}^{m}\left(t_{i}^{m}+\tau\right)=\tau\right)$ and the additional variable $\sigma_{i}^{m}(t)$ is discarded. We consider Poincare sections of the system at times the first oscillator resets. At that time the system is fully described by the phase of the second oscillator $\phi_{2}$ and all the spike variables that are currently active: $\left\{\left\{\sigma_{1}^{m}(t) \mid m \in\{1,2, \ldots\} \wedge t-t_{1}^{m} \in[0, \tau]\right\} \vee\left\{\sigma_{2}^{n}(t) \mid n \in\{1,2, \ldots\} \wedge t-t_{2}^{n} \in[0, \tau]\right\}\right\}$.

To estimate the maximal Lyapunov exponent for each value of the parameter $\tau$ a trajectory was simulated with otherwise default parameters and initial conditions. The initial part of the trajectory including the first 1000 Poincaré sections was discarded to ensure that the trajectory is sufficiently closed to any presumed chaotic or periodic attractor. The state reached by the 1001th Poincaré section was taken as 


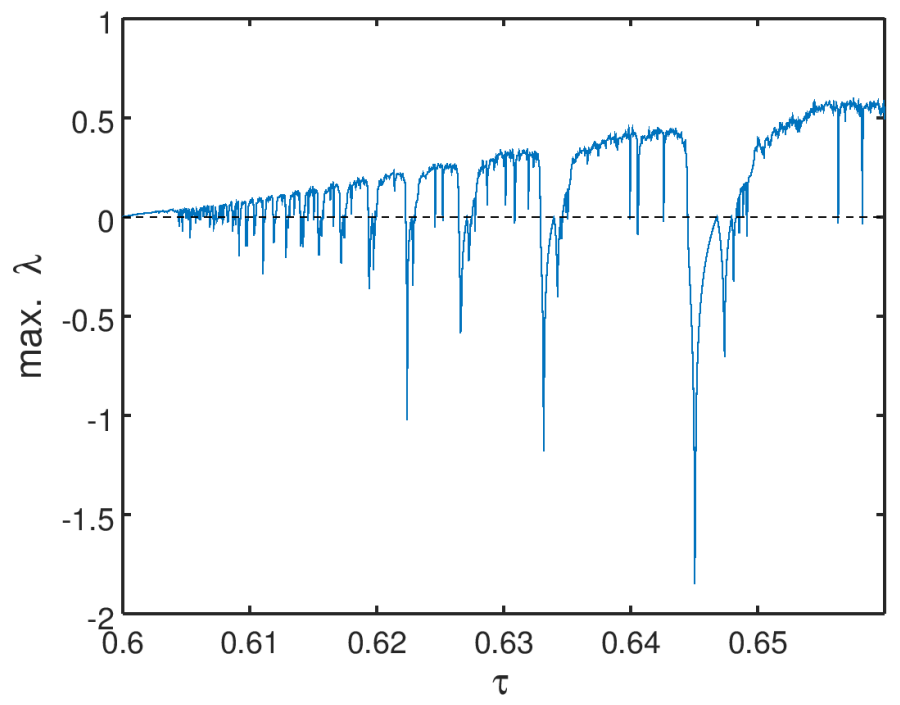

Figure 5.5: Estimated maximal Lyapunov exponent depending on $\tau$. Across $\tau$ windows with negative and positiv maximal Lyapunov exponent can be observed, corresponding to the windows of periodic and aperiodic behavior observed in the orbit diagram (figure 5.4). For details on the estimation method refer to the main text.

initial state, an initial perturbed state was obtained by distributing a perturbation of size $\Delta_{0}=10^{-10}$ equally among all active dimensions ( $\phi_{2}$ and the potentially active additional dimensions $\{\sigma\}$. The following procedure was performed iteratively for 200 steps: The perturbed and unperturbed trajectory were evolved until the first oscillator resets again. The distance between the two trajectories was measured with the 1-Norm: $\Delta_{a}=\sum_{i}\left|\delta_{i}^{a}\right|, a$ is the index of the iteration step, index $i$ goes over all active dimensions and $\delta_{i}^{a}$ denotes the difference within each dimension. While it is theoretically possible that the evolved perturbed and unperturbed trajectory live in spaces of different dimensionality, this case never occurred in our simulations, the sequence of events between both Poincaré sections was always identical. It could be seen as problematic to calculate 1-Norm distances in a space spanned by dimensions of different types. However, since all variables evolve with the same speed in time $(\dot{\phi}=1$ and $\dot{\sigma}=1$ for any $\sigma$ ) they may be regarded as comparable. Further it may be seen as problematic, that the distances in different iteration steps was measured in spaces of different dimensionality. If the dimensionality is higher, the expected distances may be larger. As last part of each iteration step, the distance between the perturbed and unperturbed trajectory was renormalized to the initial perturbation size $\Delta_{0}$ in the following way: for all active dimension $i \delta_{i}^{\prime a}=\delta_{i}^{a} \frac{\Delta_{0}}{\Delta_{a}}$ to obtain initial conditions for the next iteration step. The maximal Lyapunov exponent was estimated with

$$
\lambda_{\max } \approx \frac{1}{200} \sum_{a=1}^{200} \ln \left(\frac{\Delta_{a}}{\Delta_{0}}\right) .
$$

Figure 5.5 shows the estimated maximal Lyapunov exponent for a range of parameter 

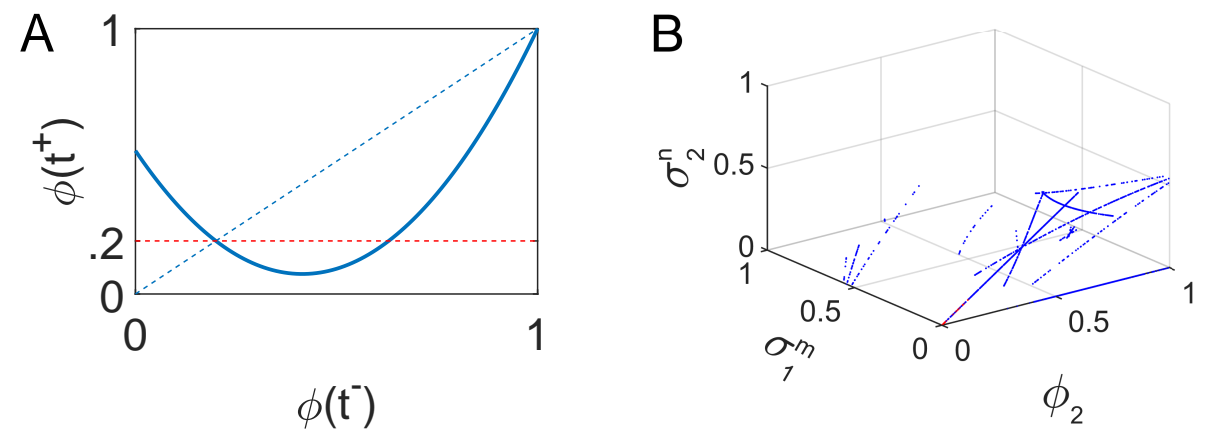

Figure 5.6: The effect of spike reception and the chaotic attractor at Poincaré sections. Panel A: The phase $\phi$ directly after spike reception is depicted depending on the phase directly before spike reception. Panel B: Poincaré sections of the chaotic attractor at resets of the first oscillator projected into the three dimensional space spanned by the dimensions $\phi_{2}$, $\sigma_{2}^{n}, \sigma_{1}^{m}(m, n$ are the spike count of spikes sent by the first and second oscillator until now). If a dimension is not active, the point is depicted at zero. Points, that live in the space with a second spike sent by the second oscillator $\left(\sigma_{2}^{n-1}\right)$ active are depicted in red. Default parameters are used.

values of $\tau$. Windows of positive and negative Lyapunov exponents correspond well to aperiodic and periodic windows observed in the orbit diagram (see figure 5.4).

\section{Maximal Dimensionality and Chaotic Attractor}

How does the attractor of the system within the chaotic regime look like? Before we can answer this question we need to discuss the dimensionality of the system. The current state of the system at any point in time is fully specified by the phases of both oscillators and the times of all spikes sent within the past of length $\tau$. In the previous section we introduced dynamic variables $\sigma_{i}^{m}(t) \in[0, \tau]$ that capture the additional dimensions introduced by spiking events. The dimensionality of the system is restricted, because there exists a minimal time between resetting and reaching threshold again in each oscillator, the minimal interspike-interval. Generally this minimal time is difficult to estimate [7], but because of the specific system set-up it can be estimated quite easily: Figure 5.6 A depicts the phase $\phi$ directly after spike reception depending on the phase directly before spike reception $\left(\phi_{\text {post }}=\phi_{\text {pre }}+\varepsilon Z\left(\phi_{\text {pre }}\right)\right)$. The part of the function above the diagonal (indicating no phase change) corresponds to an excitatory effect and below an inhibitory effect of spike reception, respectively. To find the minimal time between two resets, let us construct the optimal spike train to reach the next reset in the shortest time possible. From the set of all theoretically possible spike trains all those can be discarded as possible candidates that include a spike with inhibitory effect as last spike, since they will be less optimal compared to their exact counterparts except without the last spike. Therefore potential candidates as optimal spike trains are reduced to spike trains ending with a spike with excitatory effect and the empty spike train. The latter is not the optimal spike train, since it 
is slower than e.g. any spike train with a single excitatorily acting spike. Hence, the optimal spike train ends with an excitatorily acting spike.

The time until reaching threshold can be divided into a first part, the time during which spikes are received and a second part, the time after receiving the last spike to threshold crossing. The minimal time that the first part requires is zero and occurs if either no spike is received at all or all spiking events occur at the instant directly after reset. The minimal time that the second part requires is the time the oscillator needs from the phase that can be reached maximally by a spike with excitatory effect to reach the threshold at $\phi=1$.

In the specific system at hand with default parameters both cases occur together, if the reception of a single spike directly after reset is considered. In this case the first part requires no time. A spike reception directly after reset leads to the phase that can be maximally reached through a spike with excitatory effect, which is $\phi=0.54$. Therefore the minimal inter-spike-interval is obtained as $T_{\min }=1-0.54=0.46$.

Since 0.46 is the minimal inter-spike-interval, there can only be two spiking events in the past of length $\tau$ of each oscillator. This gives maximally six dimensions, four stemming from spiking events $\left(\sigma_{1}^{m}, \sigma_{1}^{m-1}, \sigma_{2}^{n}, \sigma_{2}^{n-1}, m\right.$ and $n$ indicating the count of the last spike sent from the first and second oscillator) and two from the two phases of the oscillators $\left(\phi_{1}\right.$ and $\left.\phi_{2}\right)$.

The state of the system at Poincaré sections only requires four dimensions to be fully described, since at reset times of the first oscillator only a single active spike sent by this oscillator can be in the system: $\phi_{2}, \sigma_{1}^{m}, \sigma_{2}^{n}, \sigma_{2}^{n-1}$.

Figure 5.6 panel B shows a three dimensional projection of 2000 consecutive Poincaré sections of the chaotic attractor. Poincaré sections, that require the fourth dimension are depicted in red. The initial part of the trajectory including the first 300 Poincare sections was discarded to ensure that the trajectory is close to the presumed chaotic attractor. The Poincaré section of the attractor seems to live in a space of dimensionality close to 1 , however, no further systematic analysis was performed.

\subsection{Source of Chaotic Behavior}

Figure 5.4 shows windows with periodic and aperiodic behavior across the dimension spanned by the parameter $\tau$. The diagram indicates a period-doubling route to chaos [37, 46, 82].

The aim of this section is to provide a better understanding of the mechanism behind the observed behavior.

Figure 5.6 panel B shows a three dimensional projection of the chaotic attractor at Poincare sections. We observe that several points of the attractor fall onto the axis corresponding to the dimension of phase of the second oscillator $\phi_{2}$. This suggests that the system at Poincaré sections returns again and again to a one dimensional state that can be described by the phase of the second oscillator $\phi_{2}$ only, all other spike induced dimensions are not active, there is no traveling spike in the system. It may be possible to develop a one dimensional map linking each of these states to the next occurrence of such a state. While this is not necessarily possible for all theoretically possible states of this kind for all parameters, because for some parameters and initial conditions the system may not return to such a state, it may still be possible for a broader parameter 

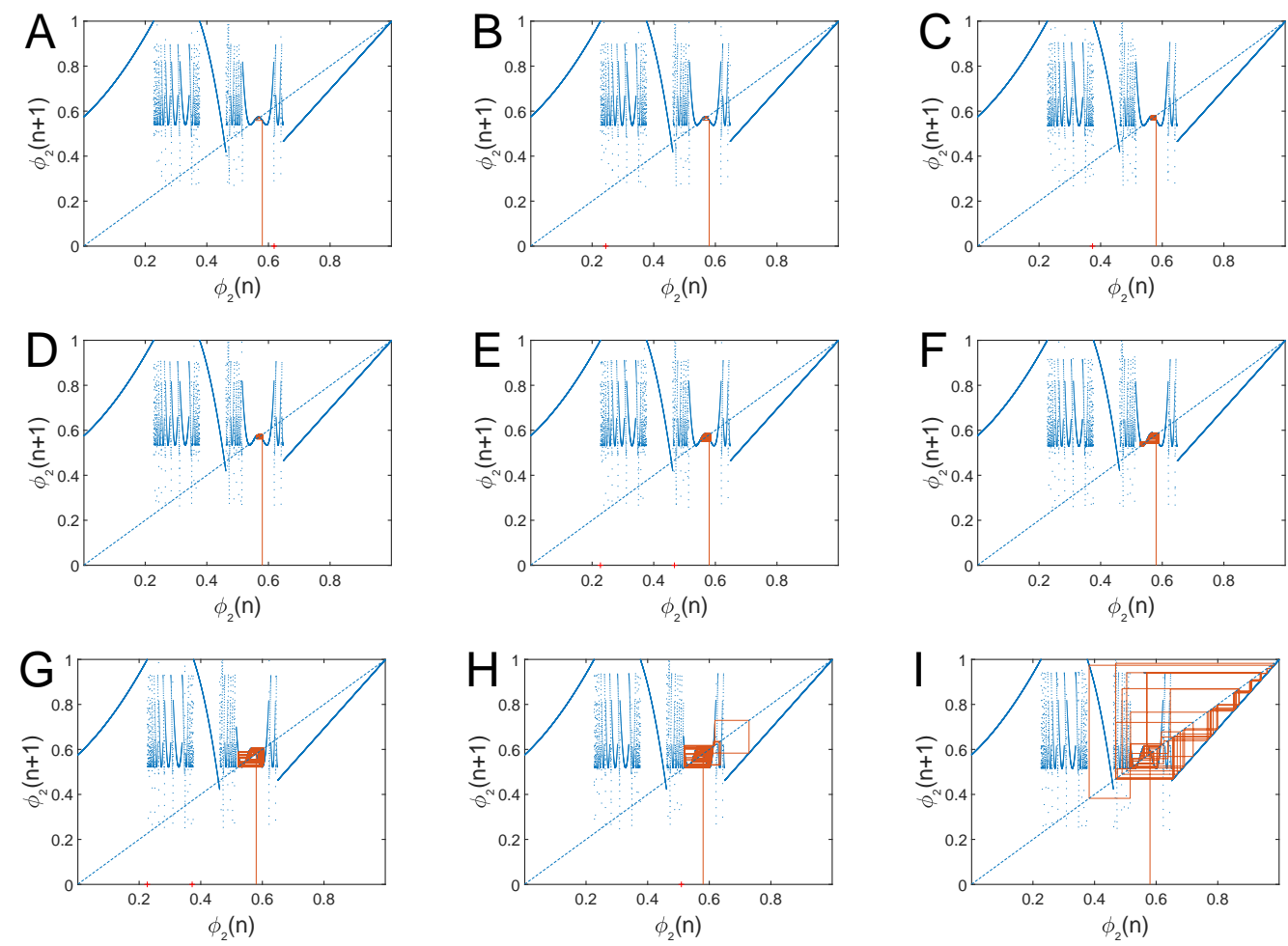

Figure 5.7: One dimensional return map shows transition to chaos similar to the transition observed in the logistic map. The one dimensional return map (blue) connects reoccuring system states characterized by no active spike variables at reset of the first oscillator. These states are fully described by the phase of the second oscillator $\phi_{2}$. The map was obtained by systematically varying $\phi_{2}$ and simulating the system for 30 resets of the first oscillator. If within this time the system never returned to the above defined system state, this is indicated by a red cross at zero. A cobweb plot of a trajectory starting at default initial conditions is shown for 90 returns. While within panels A-E the trajectory is confined within an unimodal curve segment, in panels F-I the trajectory leaves these regional confinements until in panel I the trajectory moves through most of the space. Figure 5.8 displays an enlarged view of the region characterized by the unimodal curve segment. Parameters: Standard parameters and $\tau \in$ $\{0.647900,0.648000,0.648255,0.648304,0.648550,0.648750,0.649450$, $0.649750,0.650000\}$ 

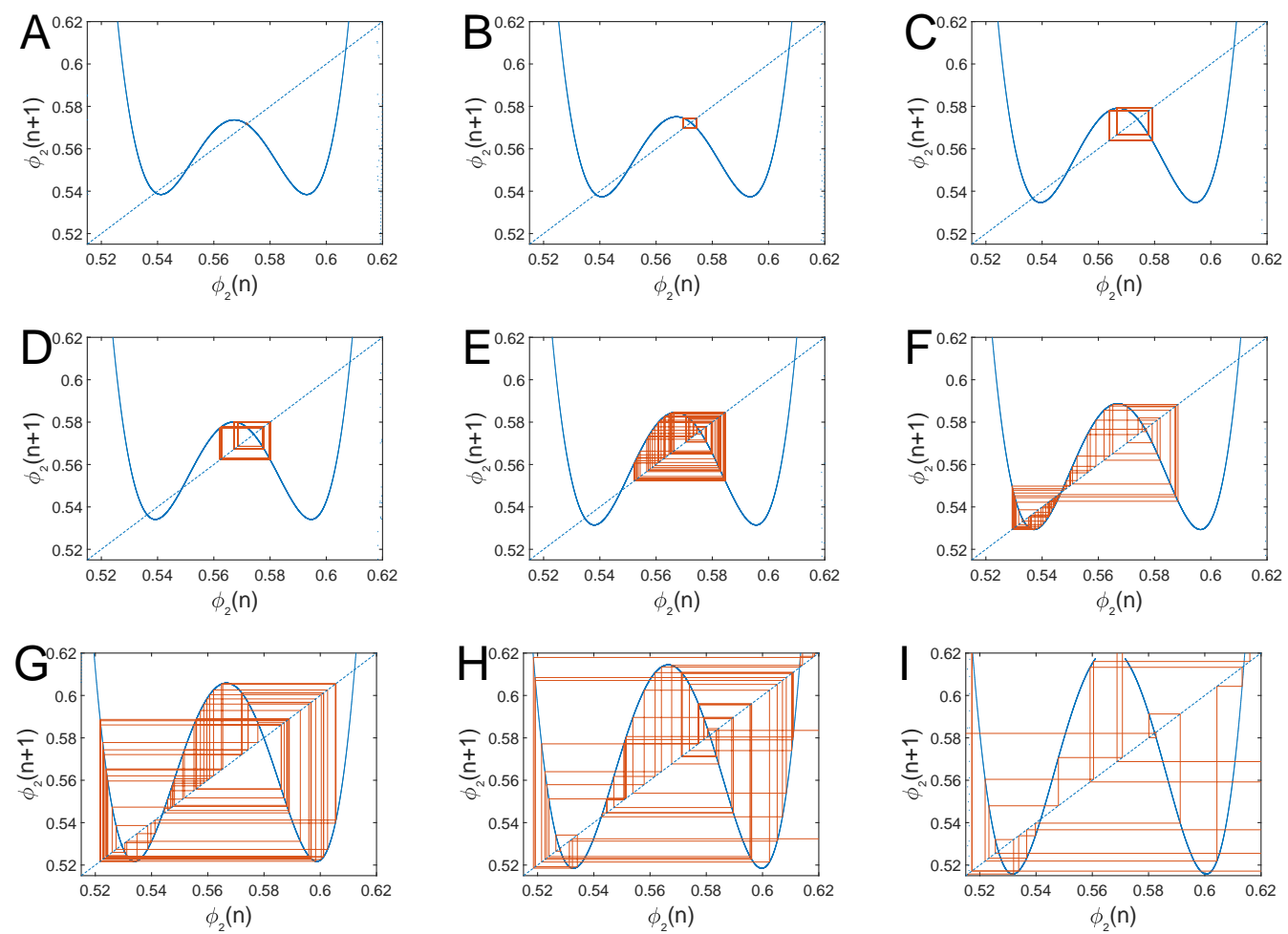

Figure 5.8: Enlarged view on the region of interest of figure 5.7 shows the transition to chaos more clearly. The first 30 returns of the trajectory were discarded, only the remaining 60 are plotted. Analog to the transition to chaos in the logistic map [54] a period doubling mechanism starting with a single fixed point (panel A), a cycle (panel B), a double cycle (panel C) and a cycle of length 4 (panel D) can be observed until in panel $\mathrm{E}$ the trajectory appears to behave fully irregular. In the following panels (F-I) the trajectory leaves the confinements of the unimodal part of the return map and extends into more and more space. 
range and most initial conditions to numerically find the corresponding return state and provide a way of insight into the system without loosing information. If such a map can be obtained the system dynamics can be understood with the theory of one dimensional maps.

Figure 5.7 shows such a map for several values of $\tau$. The values of $\tau$ were chosen to correspond to the period-doubling region displayed in figure 5.4 panel $\mathrm{B}$. The numerically obtained map is depicted in blue, a cobweb diagram showing several steps of a trajectory with default initial conditions is superimposed in orange. Since for most of the panels in figure 5.7 the trajectory is confined to a small region, this region is depicted enlarged in figure 5.8. In most parts of the space spanning from $\phi=0$ to $\phi=1$ the map is rather complicated with many sudden discontinuous jumps. However, there is a region roughly between $\phi=.5$ and $\phi=6.5$ where the map is a unimodal map. Through increasing $\tau$ the curve moves up. The trajectory, initially going to a fixed point, shows period doubling, first approaching a 2-cycle, then a 4 cycle and so fourth, until eventually the trajectory moves around chaotically, this transition resembles closely the transition to chaos in the logistic map [54] and other unimodal maps [82].

When $\tau$ is increased further beyond $\tau=0.648750$ the trajectory leaves the strict confinements of the unimodal region, but remains within adjacent space. Eventually, when increasing $\tau$ even further another transition occurs, the trajectory escapes the subregion and spans most of the space between $\phi=0$ and $\phi=1$, moving the system from a local chaotic system to a chaotic system on a global scale.

In this section we have shown that, similar to many other chaotic systems, the chaotic behavior, at least in parts, can be explained by it stemming from a unimodal return map.

\subsection{Discussion}

Which feature is responsible for the observed chaotic behavior? In this chapter we presented a coupled cell network of two units, which are coupled via exchanging delayed $\delta$-pulses, that shows chaotic behavior. Delayed $\delta$-pulses are a common feature of neural models. Hence, our system contributes to the question whether the chaotic dynamics often observed in larger spiking neural networks may arise due to the $\delta$-pulse coupling.

Several features of the system presented here may be essential to produce the chaotic behavior. So the question arises, which of these features are necessary for the chaotic behavior and which are not. These features include: Symmetry, delay and the shape of the phase response curve.

The system we studied is fully symmetrical, a property that can give rise to interesting dynamical properties in a system (see previous chapter 4). As further discussed in the next chapter, symmetries impose more constraints on possible phase space topologies, so it is unlikely, that symmetry is essential to produce chaotic behavior, which is more likely to occur with less restrictions on trajectories in phase space. Further, there are examples in the literature of low dimensional systems that show chaotic behavior only without network symmetry (e.g. [12]). Therefore the symmetrical set-up in the system presented in this chapter most likely is not a necessary requirement for 
chaos.

We chose to study $\delta$-pulse-coupling with delay. Delayed systems can show very complex dynamics, even when they only have one dynamical variable [79]. These rich dynamics in delayed systems with state dependent coupling (e.g. a system described by a delayed differential equation as such: $\dot{x}=f(x(t), x(t-\tau))$ generally is infinite dimensional, the future of the system depends on all past values within the range of the delay, which are infinitely many. But the situation in a delayed $\delta$-pulse-coupled system is different: the additional dimensions in a pulse-coupled system including systems with delayed $\delta$-pulse-coupling arise from the times when pulses were sent in the past, which in the case of delayed $\delta$-pulse-coupling only includes pulses that were sent within the past of the length defined by the delay. It is true that this increases the dimensionality of the system, but the dimensionality typically remains finite. In the system presented in this chapter, we showed that the dimensionality of the system is maximally six dimensional, while most of the time the dimensionality is even less. However, it appears to be true that the chaotic behavior observed in this chapter arises also because of these additional dimensions.

The shape of the response curve in this chapter is quite peculiar. It does not start at zero for $\phi=0$, but it ends at zero for $\phi=1$. This induces a discontinuity, since the phases $\phi=0$ and $\phi=1$ are neighboring in the periodic phase variable. It is unclear whether this discontinuity contributes to the chaos observed in this chapter. Another peculiar property of the phase response curve is its zero crossing. This results on incoming pulses having a phase advancing or phase delaying effect, depending on the current phase of the receiving oscillator. Whether this effect is necessary to produce chaos is not clear. It surely increases the irregularity of the behavior, if phases can be shifted both ways. Also common neural models often consider only phase advancing or phase delaying interaction between two neurons, while here we have mixed interactions. However, Hansel 31 report a phase response curve with a zero crossing in coupled Hodgkin-Huxley neurons.

Are $\delta$-pulses alone sufficient to produce chaotic behavior? Xu [95] reported chaotic behavior in a periodically $\delta$-pulse driven harmonic oscillator. But Laing [45] reports a case, when replacing the continuous coupling with $\delta$-pulse-coupling removes the chaotic behavior, even though making the coupling function more pointy leaves the chaotic behavior intact. The dynamic implications of instantaneous $\delta$-pulses for chaotic behavior seem unresolved.

\section{Chaos with Only a Few Neurons}

We are interested in the fundamental dynamical properties of common neural features such as delayed $\delta$-pulse coupling from a dynamical systems' perspective. Hence, our prime interest lies in the dynamics that can arise in simple systems including this feature. We did not aim for neuronal realism.

However, since we consider the work to be relevant for theoretical neuroscience, the question of how many neurons are necessary to produce chaotic behavior seems very close and interesting.

Generally researchers are interested in choosing the simplest neural model grasping core aspects of neural behavior while still being able to produce the phenomenon to be explained. Models can vary immensely within the space spanned by the dimension 
abstraction vs. biological realism and the dimension simplicity vs. complexity. Each model in this space may be the ideal model for a specific phenomenon of interest, that requires explanation. Therefore, a more refined question is: Given a certain neural model, how many neurons are necessary for chaos to occur?

In the more complex or detailed models chaos can already be found within the dynamics of a single neuron, since the dynamics of the neuron itself are high dimensional. On the other hand extremely simple neural models, such as discrete time rate models, may allow for chaotic behavior with only a few neurons, since their dynamics are described by maps, which require only one dimension to be chaotic (e.g. as in the logistic map [54]).

Examples for chaos within a single more complex neuron include the four dimensional Hodgekin-Huxley model: Doi et al. [19] report chaotic dynamics, even though within a nonstandard parameter regime.

Another example of chaos within a single rate neuron with unusual periodical driving is found by Kürten and Clark [42], the complete autonomous model including the neuron with an adaptation mechanism and the periodic driving consists of four ODEs.

Ermentrout [21] presents a FitzHugh-Nagumo oscillator consisting of a voltage variable and a recovery variable resembling potassium dynamics. Introducing another variable that can be understood as mimicking another inward conductance, leads to chaotic behavior, the complete system simulating one neuron is fully described by three ODEs. Furthermore, he shows that adding an excitatory interneuron to a Wilson-Cowan system consisting of an excitatory pyramidal cells and an inhibitory interneuron, has very similar dynamical equations and also shows chaos, an example of a chaotic system of three neurons.

Time discrete rate neurons with synchronous update are situated further towards the simple and abstract side of neural models. At each time step the new rate value of each neuron is updated by passing the sum of all presynaptic rate values through a sigmoidal activation function. From a mathematical perspective these models are multidimensional maps. Renals and Rower [70] did a broad parameter search for these kinds of models and never found chaotic behavior for networks with two or three neurons. However, Wang [90] presents a system class of only two such neurons and by showing that its dynamics are topologically conjugate to S-unimodal maps, demonstrates that the dynamical behavior is chaotic for certain parameters.

Examples for chaos in neural networks with only a few neurons, which are modeled neither as very simple nor very complex include the following:

Laing [45] considered symmetrical all-to-all coupled theta neuron networks with and without self-coupling. The dynamics of each neuron is fully characterized by a single ODE. The author finds chaos in networks of three and four neurons. This may at first be counter intuitive, since due to the network symmetry the neurons also show order conservation. However, these two properties are not in conflict with each other (see previous chapter 4 regarding order conservation in symmetrical networks as well as reference [11] for another such example).

Marcus und Westervelt [52] consider leaky integrator neurons. The delayed inputs are summed and passed through a sigmoidal transfer function. Each neuron therefore is described by a single delayed differential equation. They report chaotic behavior in networks of three neurons. 
In this chapter we are concerned with delayed $\delta$-pulse coupling. None of the above examples includes chaotic behavior with $\delta$-pulse coupling (but see Laing [45], who reports that chaos in his systems vanishes if the coupling is replaced by instantaneous $\delta$-pulses). Even though we proposed a system that is not aimed to be a realistic neural model, the system's mathematical structure is identical to neural phase oscillators with delayed $\delta$-pulse coupling, which is multiplied by a phase response curve. E.g. the leaky-integrate-and-fire neuron, a widely applied neuron model, often falls into the same system class. Therefore we think that we have contributed towards answering the question on how many neurons are necessary to produce chaotic behavior within this system class.

\subsection{Summary}

In this thesis we want to understand the dynamical implications of common neural features. In this chapter we considered delayed $\delta$-pulse-coupling and found that chaotic behavior is possible in a system of only two phase oscillators. The system was studied numerically, many of the typical signatures of chaos were found and we were able to show that the chaotic behavior is related to chaos arising in unimodal maps. The system in this chapter provides another example of a dynamical phenomenon occurring in a pulse-coupled system, that does not occur in a corresponding ODE system. Because of the Poincaré-Bendixson-theorem chaos in an ODE network requires at least three network nodes, while the network here only has two nodes. Another example for a qualitative difference between ODE and pulse-coupled systems was presented in the previous chapter. 



\section{Chapter 6}

\section{Discussion}

The goal of this thesis is to understand the fundamental properties of collective dynamics emerging from basic dynamical features rarely considered in classical physics but commonly used in models of systems in biology and, in particular, neuroscience. Specifically, we take a dynamical systems' perspective and ask which phenomena may occur in the presence of adaptation and (possibly delayed) pulse coupling.

Taking a dynamical systems' view, when we ask about effects, we refer to the topology of phase space, its basins of attraction, basin boundaries, special trajectories. We want to know whether phenomena such as chaos, limit cycles or more involved dynamics can be generated with the help of these features and how they can be generated. We are interested in understanding the mapping between the equations defining the temporal evolution of the system and the corresponding phase space topologies.

There may be no systematic way of understanding which effects emerge from certain dynamical features introduced to the equations defining a dynamical system in general. Which general statements can be made about the phase space topology stemming from introducing certain features?

While it is hard to provide statements about the global phase space portrait, dynamical features impose limitations on how trajectories can interact locally. For instance, given a certain system type or feature, can trajectories merge or split, do they need to be continuous or can they jump over one another? Does the system have 'hidden' dimensions, such that while trajectories are continuous and cannot cross each other, they appear to cross each other in the lower dimensional projections onto the space spanned by only the dynamic variables considered 'natural'.

Rather than attempting a general systematic study, we here address these problems from the point of view of minimal models and ask whether and how a given overall system dynamics may arise from a given feature added to idealized model systems.

In the following section we will expand on the considerations regarding freedom and limitations of trajectories in phase space. We do not aim at providing a complete analysis, rather, we provide a first attempt to group dynamical systems coarsely by their restrictions on trajectories and refer to example systems illustrating these points. 


\subsection{Theoretical Considerations on Restrictions and Freedom in Phase Space}

\section{Smooth Ordinary Differential Equations as Reference Systems}

Our starting point is a smooth system of ordinary differential equations. Such a system has the restrictions that all trajectories are necessarily continuous. Further the existence and uniqueness theorem holds (see chapter 2), which means that trajectories cannot join or split. It also means that the past and future of each trajectory is unique: Given a certain point in phase space, the full future and full past is uniquely defined by the trajectory passing through this point.

\section{Adding Restrictions - Introducing Symmetries}

It is possible to introduce more restrictions on the trajectories in phase space. An example is the introduction of symmetries. Introducing symmetries often yields interesting dynamics [8, 11, 24, 30, 62, 81, 85]. Symmetries may induce flow invariant subspaces, which act as barriers to the flow. If a trajectory is on such a subspace, it will remain on this space forever. It also means that if these invariant subspaces fully separate the space into volumes, it is not possible for a trajectory to pass from one side to the other. This is because crossing the border means intersecting with the trajectories on the flow invariant subspace and this would be in direct violation with the uniqueness guaranteed of the existence and uniqueness theorem. Whereas the above reasoning applies for smooth ODE systems, introducing certain features into the dynamical equations grants trajectories more freedom or removes conditions, such that the existence and uniqueness theorem does not apply. We will introduce some such examples below. For a more detailed account see reference [25] and the discussion section of chapter 4 .

\section{Removing Restrictions: 1 - No Uniqueness of Flow}

There are several restrictions on trajectories in smooth ODE systems, that can be removed allowing for richer dynamics. For example the system can be changed in a way that allows for trajectories to merge or split. This may be the result of different dynamical features, which are introduced into or altered in an ODE system. An example is the system presented by Dixon et al. [18. They present a system of two ordinary differential equations. While the dynamical equations are smooth almost everywhere there is a singularity where they are not. It turns out that almost all trajectories in the vicinity eventually merge into a single trajectory at this point. Therefore the system has no time reversal in the sense that points on this merged trajectory do not have a uniquely defined history. An interesting consequence of the specific phase space structure is its strong numerical instability. When simulated the system appears to be highly chaotic.

\section{Removing Restrictions: 2 - Introducing 'Hidden' Additional Dimensions}

Another way to remove constraints on phase space topology is achieved by allowing trajectories to pass through additional 'hidden' dimensions. An ODE system of $k$ 
dynamic equations describes the evolution of $k$ dynamic variables. However, there are several features that can be introduced into such a system, that give it the appearance of being $k$ dimensional, even though the system is actually higher dimensional. Here, we will provide a few examples:

Explicit time dependence. Consider the following non-autonomous system:

$$
\begin{aligned}
& \dot{x}_{1}=f_{1}\left(x_{1}, x_{2}, t\right) \\
& \dot{x}_{2}=f_{2}\left(x_{1}, x_{2}, t\right)
\end{aligned}
$$

This system has two dynamical variables and is described by two differential equations. However, to specify the state of the system, the value of $x_{1}, x_{2}$ as well as the time point $t$ is required. Therefore, the system is actually three dimensional. This becomes obvious, when the system is rewritten in the following way:

$$
\begin{aligned}
& \dot{x}_{1}=f_{1}\left(x_{1}, x_{2}, x_{3}\right) \\
& \dot{x}_{2}=f_{2}\left(x_{1}, x_{2}, x_{3}\right) \\
& \dot{x}_{3}=1 .
\end{aligned}
$$

Through this substitution the explicit time dependence is dropped, instead a third dynamical variable is introduced, that evolves with unity time. The evolution of the two dynamical variables $x_{1}$ and $x_{2}$ is conserved under the substitution. An example of richer dynamics in systems with explicit time dependence compared to systems without are driven two dimensional oscillators. Because the explicit time dependence adds a dimension, these systems can be chaotic without violating the Poincaré-Bendixson theorem. As an example see the system studied by Moon and Holmes [60. They study a ferroelastic beam, that shows oscillatory bending between two magnets. The dynamics of the system can be reduced to a second order non-autonomous differential equation that can be understood as a forced double-well oscillator: A damped particle in a double well experiencing sinusoidal forcing changes chaotically between the two wells.

Systems with noise. Real world phenomena are often modeled through dynamical systems. The dynamical variables considered may only be very few. However, increasing the resolution of almost any real world systems will lead to a highly complex picture of an endless amount of entities interacting. E.g. any macroscopical object is made up of a vast amount of molecules, which constantly interact with molecules surrounding the object. For most research questions explicitly modeling all these entities is neither feasible nor elegant. Instead the micro interactions with the environment are assumed to be chaotic and modeled by introducing noise into the dynamical equations. Therefore introducing noise is a way to account for the extreme high dimensionality of a real world system in a simple model. The following equation is an example of a stochastic differential equation, the time evolution of the dynamic variable $x$ is a function of $x$ and gaussian white noise $\xi(t)$ :

$$
\dot{x}=f(x)+\xi(t)
$$


The structure of this equation has much in common with a non-autonomous differential equation. While there is a part of the equation that depends on the dynamic variable $x$, there is also a part that depends explicitly on the time $t, \xi(t)$. The stochastic influences can be understood as external input to the system, a form of explicit time dependence. To describe the full state of the system only the values of the dynamic variables are required. At the same time, two systems with identical initial conditions evolve differently unless the noise is realized in exactly the same way. Therefore, to fully specify the system the realization of noise at all times is necessary. Approached from this angle, the system may be viewed as infinite dimensional, since infinite knowledge is required to create identical outcomes of two systems with identical initial conditions. If systems with different noise realizations are compared, then trajectories can cross each other in phase space. Whether further restrictions are removed depends on the specific form of noise used, e.g. allowing for jumps in the trajectories by introducing discontinuous noise.

Systems with delay. Delayed systems are an interesting case of introducing 'hidden' additional dimensions. The family of delayed systems is quite diverse (e.g. see [28]). As an example we briefly consider the following system:

$$
\dot{x}=f(x(t), x(t-\tau)) .
$$

The change of the variable $x$ depends on the current value of $x$ through $x(t)$ as well as the value that $x$ had $\tau$ time units in the past. This system has one dynamical variable, which is $x$ and it is described by a single dynamical equation. It appears to be one dimensional. However, to uniquely define the system's initial conditions the current value of $x$ has to be provided as well the whole history of $x$ up to $\tau$ time units into the past, since this part of the history of $x$ still influences the future of the system. For a time continuous variable $x$ the set of $x$ values included in this history is infinite dimensional and so is the system. As a result trajectories passing through the same point in the space spanned by the dynamical variables can evolve from there quite differently depending on their unique past. Trajectories in this space therefore can cross each other. There are many examples of time delayed systems with one variable showing very rich behavior(e.g. the system $\dot{x}=\sin (x(t-\tau))$ shows chaos [79]).

\section{Removing Restrictions: 3 - No Continuity of Trajectories}

A system of ordinary differential equations has continuous trajectories in phase space. Also this restriction can be broken by introducing certain dynamical features or considering time discrete systems. Here we will briefly discuss the latter.

Time discrete systems. A time discrete system has the form

$$
\mathbf{x}_{\mathbf{t}+\mathbf{1}}=\mathbf{f}\left(\mathbf{x}_{\mathbf{t}}\right) .
$$

From a theoretical perspective this set of $k$ equation is a $k$-dimensional map. If we consider the space spanned by the variables, the analogue to the phase space of a time continuous system, then trajectories are sets of generally unconnected points. If 
the points are plotted in temporal order, then the next point may appear at a very distant place from the current point and the structure behind the time evolution is difficult to grasp. Hence, to understand the behavior of maps other forms of graphical representation are commonly used (e.g. see the cobweb plots in chapter 5 as an example). Allowing such jumping within the value set of the dynamical variables can give rise to very rich behavior even in low dimensional systems. A very famous example is the logistic map $\left(x_{t+1}=a x_{t}\left(1-x_{t}\right)\right)$, which displays rich and chaotic behavior for a range of parameter values $a$, even though the map is one dimensional (see [54]).

Hybrid systems. Sometimes a real world system is best modeled by a dynamical system that combines time continuous and time discrete dynamics. An example system is provided by Nakano and Saito [61]. They describe a system of two dynamic variables that evolves continuously in time until a predefined condition is met. At this point the state of the system is mapped to another state and the continuous time dynamics continue from the new state. While a hybrid system does not necessary need to have discontinuous trajectories in the space spanned by the dynamic variables, discontinuities can be introduced through a map capturing the time discrete part of the system's evolution as done in the system of Nakano and Saito. For an example of a continuous hybrid system see reference [65].

\subsection{Dynamical Neural Features and Phase Space Constraints}

Where do the features studied in this thesis fit into the above classification scheme?

Feature 1: adaptation. There is no general definition of how a dynamical system needs to be set up to be considered adaptive. We decided to consider systems that shows an ongoing minimization of an energy functional, which itself may change on a slower time scale, since this behavior is common to adaptive systems. There are many system types compatible with these requirements. We studied a system that can be described by two smooth ODEs. Therefore the existence and uniqueness theorem holds, trajectories cannot cross each other and are confined into a two dimensional space, the set of possible topologies is restricted as described by the Poincaré-Bendixson theorem.

Feature 2: pulse-coupling. Pulse-coupling falls into the above category of introducing hidden dimensions. To characterize the current state of a pulse-coupled system fully, it is necessary to define the current values of the dynamical variables, but also all the time points, when pulses were sent in the past, that still influence the future of the system. However, an exception are instantaneous $\delta$-pulses, since they do not extend in time and therefore do not introduce additional dimensions.

Pulse-coupling is a very peculiar feature, since it dynamically changes the dimensionality of the phase space itself. The consequences of this behavior are not well understood: Is it meaningful to ask whether neighboring trajectories can merge, if the evolution of one of the two systems may increase the dimensionality of the space in the next moment, so that the two trajectories suddenly are not part of the same 
space any more? Does it even make sense to talk about a trajectory, that is part of a space with temporally changing dimensions? Nevertheless, the space spanned by only the dynamic variables, not including the additional dimensions created by pulses, remains intact throughout the whole evolution of the system. It therefore seems to be meaningful to compare trajectories in this subspace, even if they really live in spaces of different dimensionality. Though care has to be taken, since only a subspace is considered, trajectories can merge or split and cross each other.

Pulses can have very different shapes. They can be discrete $\delta$-pulses, continuous or smooth. They can be constructed in a way that ensures that the trajectories in the space spanned by the dynamic variables are also smooth [38. The shape of the pulse also influences which topologies are theoretically possible in the space spanned by the dynamic variables, e.g. $\delta$-pulses produce jumps.

Combining features. It is interesting to consider what topologies can occur, if different features are combined. In chapter 4 pulse-coupling and symmetry were both introduced into the same system. While symmetries often lead to further restrictions in phase space as discussed above, combined with pulse-coupling this is not necessarily the case. For a detailed discussion on pulse-coupling in symmetrical systems, see the discussion section of chapter 4 .

\subsection{The Approach of Studying Example Systems}

While the above considerations provide an idea of which structures might be possible for a specific system class they provide very little knowledge of how the phase space topology of a specific system actually looks like or how a specific system should be constructed to produce a certain phase space structure. We have reduced the set of possible topologies but we do not have a one to one mapping between system class and phase space topology.

To give an example, considering a non-autonomous system of two dynamical variables effectively describes a three dimensional system. Having at least three dimensions in a continuous smooth system is a necessary condition for chaos to occur but not sufficient. Hence, knowing that non-autonomous systems with two dynamic variables are three dimensional opens up the possibility for such a system to be chaotic, but it does not mean that every such system indeed shows chaotic behavior. Further knowledge is required to understand what the additional conditions are for chaos to occur.

Eventually, this is a question of fully understanding the relation between systems defined by their dynamical equations and corresponding phase space topology.

Closing the gap between system definition and phase space topology. Dynamical systems have these two representations: dynamical equations and the phase space portrait. A major goal of dynamical system theory is to match these two. How do they relate to each other? This is not a statement on a specific system but on how a system class looks like to produce a set of flows in phase space that are topologically equivalent or share certain properties. The above considerations gave us some insights into what may be possible in phase space, but to know, what topologies actually stem 
from certain dynamical features, we need to change our approach. Closing the gap of understanding between phase space structure and the dynamical rules defining the evolution of a system is a central goal of dynamical system theory.

It is surprisingly hard to design a set of dynamical equations, given a specific non trivial phase space topology. At the same time it is relatively easy to produce the phase space portrait of a given set of dynamical equations, at least numerically with the help of computers (even though sometimes care has to be taken, e.g. see reference [18. Therefore, to find an answer for which system class matches which phase space topology, it seems the right approach to study many example systems in the hope that interesting behavior will occur and with enough systems patterns can be observed and principles can be deduced. Following this approach it may also be possible to discover completely new topologies, e.g. as Lorenz did when he studied his chaotic oscillator [49].

In this thesis we took this approach and studied three example systems to understand which topologies can be expected by introducing these features in general. We have found the following:

A minimal system of adaptation. In chapter 3 we introduced a minimal system of adaptation, which has a phase space portrait of nested limit cycles. A simplified version of the system was also introduced, whose phase space portrait is very similar to the portrait of the original system and which allows for proving the existence of limit cycles. This simplified system, however, is a piece wise smooth system ([10]), different regions is phase space follow different dynamical rules. While trajectories are continuous everywhere, they are not smooth. At the borders of these regions they can merge, the system shows finite time convergence and is not time reversible. This leads to a structure we called funnel structure, which is essential for the proof. When the funnels of this structure are closed the full flow is discretized, all trajectories entering the structure leave through one from a discrete set of exit points.

The system we studied is a very simple adaptive system, still we found an interesting phase space topology. Varying parameters showed that the phase space portrait is robust. It still remains to be understood what the key ingredients are to produce this topology. What does a system generally look like that is adaptive and produces such a nested limit cycle topology?

We believe that our conceptualization of adaptation from a dynamical systems' perspective is very broad and will encompass many more examples with potentially quite different phase space portraits.

For alternative perspectives on the system in the context of other research fields, see the discussion section of chapter 3 .

All-to-all pulse-coupling in a symmetrical phase oscillator network. Chapter 4 focuses on the features pulse-coupling, symmetry and the effect of self-loops in an all-to-all coupled network. Our motivation in the context of scientific literature is provided in the introduction section of chapter 4 . We are interested in whether the dynamic variables conserve their ordering and if not, how they reorder themselves. The systems studied here are based on work by Kielblock et al. [38]. The authors considered all-to-all coupled phase oscillators with full permutation symmetry. They 
demonstrated that the restrictions on such systems described by ODEs do not apply if pulse-coupling is introduced. Even if the shape of the pulses is chosen in a way that pulse reception does not induce any non-smoothness into the trajectories in the space of the dynamic variables, the oscillators do not show order conservation.

We extend their approach by gradually introducing self-loops. First we showed that introducing self-loops into the smooth pulse-coupled system studied by Kielblock et al. restores order conservation. For a theoretical discussion on how pulse-coupling circumvents the restrictions imposed by symmetry on ODE systems and why selfcoupling restores order conservation see the discussion section of chapter 4 .

Then we studied this transition in a system with delayed $\delta$-pulse-coupling. The system shows a smooth transition to breaking of order conservation when gradually removing self-loops. When order is not conserved the oscillators overtake each other. The mechanism behind this reordering process is uncovered, depending on the parameters the emerging reordering patters are simple or very complex and despite being fully deterministic resemble randomness. The mechanism involves a folding step, which is also common for chaotic systems. However, while the reordering may be quasi-chaotic, the global dynamics approach the fixed point of the synchronous state. While the basic phenomenon is identical to the smooth pulse-coupled system, it is not clear whether the mechanism behind the breaking of order conservation is identical, since $\delta$-pulses allow for jumps in trajectories and induce less restrictions on phase space structures. We argue that order conservation with self-loops in a smooth coupled system as proposed by Kielblock et al. necessarily occurs, whereas this may not be true in a $\delta$-pulse-coupled system. We conjecture that a fully symmetrical system including self-loops with delayed $\delta$-pulse-coupling should exist, that does not show order conservation. For details see the discussion section of chapter 4 .

Chaos in a system of two pulse-coupled phase oscillators. In chapter 5 we again focus on delayed $\delta$-pulse-coupling. We present a system of only two phase oscillators, that shows chaotic behavior, something that would not be possible in a system of two oscillators described by ODEs. It can be shown that at least in part, the chaos arises through a period doubling mechanism analog to chaos in a logistic map [54]. However, it is not clear what the essential ingredients are to produce the chaoticity. We used delayed $\delta$-pulses, yet potentially chaos can also occur with instantaneous $\delta$-pulses or continuous pulses. We chose a phase response curve with a zero crossing, that is discontinuous at the reset point. Would chaos be possible without any of these two properties? It is clear that more systems need to be studied that treat these ingredients separately to shed light onto these questions. For a detailed discussion, also placing the system into scientific context, see introduction and discussion sections of chapter 5 .

\subsection{Introducing Neural Features Does Not Always Change Everything}

We have seen that studying dynamical features commonly used in theoretical neuroscience can produce interesting and rich dynamics. Often these dynamics were contrasted with the dynamics stemming from analog systems of ODEs and it was 
shown that the dynamics are qualitatively very different. But sometimes the opposite is also true: changing the system class may result in a phase space portrait that still strongly resembles the original system. In chapter 3 a system described by two ODEs was introduced and its phase space portrait was determined through numerical simulations. In a second step a simplified system was introduced, one of the two ODEs was replaced by a discontinuous function. The phase space portrait remained very similar but the new form allowed for analytic treatment. In contrast, one of the main points in chapter 4 and 5 was showing that systems described by ODEs as compared to systems with pulse-coupling may show very different qualitative behavior despite their similarity in their dynamical set-up.

The following question arises: when does introducing new dynamical features into a system, which grant trajectories more freedom in phase space, substantially change the phase space portrait and when does it not? 



\section{Chapter 7}

\section{Summary}

In this thesis we aimed at understanding the fundamental properties of features commonly used in models of theoretical neuroscience from a dynamical systems' perspective. Dynamical system theory was developed while studying classical physical systems, such as planetary movements. Such systems can often be described by smooth ordinary differential equations and the dynamics arising from such equations are relatively well understood. However, in recent years the quantitative study of biological systems, including neuronal systems, has become a major research field. Models of these systems often include features that are not common to classical physics and still lack a good understanding from a dynamical systems' perspective. We contribute towards closing this gap.

In theoretical neuroscience models often encompass networks with many interacting neuronal units. These networks exhibit interesting dynamical phenomena. At the same time the neuronal units themselves may include dynamical features, such as pulse-coupling or adaptation, which may be able to produce rich dynamics already in very simple network architectures. If so it may be possible to consider extremely simple systems including these neural features and observe complex dynamics often associated with complex network models.

In this thesis we considered three very simple systems including features such as adaptation and pulse-coupling and analyzed their behavior. In all cases we found that these simple systems can produce surprisingly rich dynamics.

In a minimal adaptive system described by only two ordinary differential equations, we found a phase space portrait of presumably infinitely many nested limit cycles. In a simplified system with the same qualitative phase space portrait of nested limit cycles, we identified a structure, called funnel structure which separates the flow in finite time into a set of discrete trajectories. Further, we showed that these trajectories are cycles, hence proving the nested limit cycle behavior in a certain parameter regime and hence providing analytic insights into the global dynamics of a nonlinear system.

Building on work by Kielblock et al. [38] we analyzed symmetrical all-to-all pulsecoupled phase oscillator networks. While corresponding ODE systems necessarily show order conservation of the oscillators, this is not true if pulse-coupling is introduced. We showed that gradually introducing self-loops may restore order conservation. In a system with delayed $\delta$-pulse-coupling we were able to analytically understand the transition to order conservation and to uncover the mechanism behind the reordering process. Depending on system parameters simple or quasi-chaotic 
reordering patterns were observed. We discuss how it is possible that pulse-coupled oscillators can circumvent the dynamical restrictions present in ODE systems and break order conservation. Further, we discuss why order conservation may be restored by introducing self-loops.

Finally we described a system of only two delayed $\delta$-pulse-coupled phase oscillators, that shows chaotic behavior characterized by a beautiful orbit diagram. We found that the observed chaos can be related to the period-doubling route to chaos arising in unimodal maps, such as the logistic map.

We have seen that the neural features we focused on indeed are able to produce very rich dynamics, even if embedded into very simple systems. Studying these systems has lead to a better understanding of the dynamic properties of these features. At the same time it turns out that the features themselves comprise a collection of different subfeatures with different dynamical properties, i.e. Pulse-coupling can have quite different properties depending on the shape of the pulse used. We also encountered conceptual difficulties, e.g. what do we mean by adaptation in a dynamical context? Or how can we compare trajectories in a pulse-coupled system, if the dimensionality of the space containing them changes dynamically?

It is clear that our systems studied here are only a starting point towards gaining a full understanding of the dynamical implications of adaptation and pulse-coupling.

If more systems are studied encompassing these dynamical features hopefully patterns and principles can be discovered to develop a better theory of which topologies are possible and under which conditions. And maybe new dynamical structures are discovered altogether. 




\section{Appendix}

\section{A - A Particle Interacting with Its Potential - an Alternative Representation}

There is a second equivalent way of rewriting the original system as a particle interacting with its potential:

$$
\begin{aligned}
& V(x, c)=-\varepsilon x c \\
& \dot{c}=-\partial_{c} V \\
& \dot{x}=-A \cos (x-\varphi)-c .
\end{aligned}
$$

Rewritten this way $c$ and not $x$ is interpreted as the particles position, while $x$ is a variable that changes the potential depending on $c$. This formulation is equivalent to the one in section 3.2 .1

\section{B - A Second Order Differential Equation - an Alternative Representation}

In the main text in section 3.4 on page 37 the original system was rewritten as a second order differential equation. There is a second equivalent way the system can be expressed as a single second order differential equation:

$$
\ddot{x}=A \sin (x-\varphi) \dot{x}-\varepsilon x
$$

Interpreted as a mass hanging on a mechanical spring the last term $\varepsilon x$ acts as a restoring force with a spring constant of $\varepsilon$. The term $A \sin (x-\varphi) \dot{x}$ can be understood as a damping force, since it depends on the velocity $\dot{x}$. However, it also depends on the position $x$. Hence, the damping depends also on the current position of the mass. Through the sinusoidal function, the damping changes its sign, the resulting oscillator is an active oscillator.

\section{C - Existence of Limit Cycles - Connecting Funnel $F_{k}^{-}$with Funnel $\boldsymbol{F}_{\boldsymbol{k}}^{+}$}

To show that the trajectory leaving a funnel exit point $F_{k}^{\circ \pm}$ is a cycle we want to demonstrate that a the trajectory passes through the following sequence of states (equation 3.6):

$$
F_{k}^{\mathrm{o}+} \stackrel{(i i)}{\longrightarrow} F_{k}^{\mathrm{in}-} \stackrel{(i)}{\longrightarrow} F_{k}^{\mathrm{o}-} \stackrel{(*)}{\longrightarrow} F_{k}^{\mathrm{in}+} \stackrel{(i)}{\longrightarrow} F_{k}^{\mathrm{o}+}
$$


The sections 3.3 .3 and 3.3 .4 demonstrate transition (i), provided a certain parameter regime. In the proof sections (3.3.3) and (3.3.4) on pages 24 fff and 32 fff it was shown that the trajectory leaving funnel $k$ on the right side will reach the funnel entrance of the $k$ th funnel on the left. However the transition $\left(^{*}\right)$ showing that a trajectory leaving a left funnel $F_{k}^{\mathrm{o}-}$ will eventually reach the funnel entrance region on the right $F_{k}^{\text {in }+}$ remains to be proven. This part of the proof was left for the appendix, because it works completely analog. Here we define the corresponding variables and functions and demonstrate the proof of this remaining step.

$\boldsymbol{x}_{k}^{*+}$ - the position where the trajectory returns to $\boldsymbol{c}=-\boldsymbol{A}$. Here we study the trajectory $(x(t), c(t))$ leaving at the funnel exit point $F_{k}^{\mathrm{o}-}$ and its corresponding curve $c(x)$. From the system definition (equation 3.3) it follows that while $c<A$ and $x<0$ the trajectory is monotonically decreasing in $c(\dot{c}<0)$ and monotonically increasing in $x(\dot{x}>0)$. Eventually the trajectory passes $x=0$. While $c<A$ and $x>0$ the trajectory is monotonically increasing in $x$ and in $c(\dot{x}>0$ and $\dot{c}>0$ until the trajectory reaches $c=-A$. We denote the $x$-position of this return as $x^{*+}$.

$$
x_{k}^{*+} \in F_{k}^{\mathrm{in}+} \Longleftrightarrow x_{k}^{\mathrm{c}+}-\pi \stackrel{(i i .2)}{\leq} x_{k}^{*+} \stackrel{(i i .1)}{<} x_{k}^{\mathrm{c}+}+\pi
$$

Therefore showing the transition $F_{k}^{\mathrm{o}-} \stackrel{(i i)}{\longrightarrow} F_{k}^{\mathrm{in}+}$ is equivalent to showing that the two inequalities (ii.1) and (ii.2) hold.

Replacing the trajectory leaving at $\boldsymbol{F}_{k}^{\mathbf{0}-}$ by the function $\boldsymbol{c}(\boldsymbol{x})$. We are only interested in the part of the trajectory from leaving the funnel exit point $F_{k}^{\mathrm{o}-}$ until reaching $c=-A$ again. This part of the trajectory can be expressed through the corresponding curve $c(x)$ In the paragraph 3.3 .2 (remarks on monotonicity) it was discussed that analog to the behavior of the trajectory any connected curve $c(x)$ while $c<A$ is strictly monotonically decreasing with $x$ while $x<0$ and strictly monotonically increasing while $x>0$. Because of the monotonicity the curve $c(x)$ with $c \leq A$ is a function, there is exactly one $c$-value assigned to any $x$-value. The following part of the proof concerns the curve $c(x)$ within this region.

\section{(ii.1) To Show: $x_{k}^{*+}>x_{k}^{\mathrm{c}+}-\pi ; x_{k}^{*+}$ Lies Right of the Left Entrance of $F_{k}^{\text {in }+}$}

Defining $\tilde{\boldsymbol{x}}^{\mathrm{f}}$ and $\tilde{\boldsymbol{f}}(\boldsymbol{x})$. The function $\tilde{f}(x)$ is defined in the following way:

$$
\tilde{f}(x):=-x+\tilde{x}^{\mathrm{f}}
$$

$\tilde{x}^{\mathrm{f}}$ is defined such that it has the same distance to the funnel exit point $F_{k}^{\mathrm{o}-}$ as the left border of the funnel entrance $F_{k}^{\text {in+ }}$ from $x=0$ :

$$
\left(x_{k}^{\mathrm{c}+}-\pi\right)-0=\tilde{x}^{\mathrm{f}}-x_{k}^{\mathrm{c}-} .
$$

Now we show that with the above definition it follows that $\tilde{x}^{\mathrm{f}}<0$ :

For $0<\varphi<\pi$ :

$$
\tilde{x}^{\mathrm{f}}=2 \varphi-2 \pi<0 .
$$


For $-\pi<\varphi<0$ :

$$
\tilde{x}^{\mathrm{f}}=2 \varphi<0 .
$$

In the following we show that $x$ and $\tilde{f}(x)$ are situated within the same segment type. Both either lie within an up shifted or a down shifted segment: $s(x)=s(\tilde{f}(x))$. For $0<\varphi<\pi$ as well as $-\pi<\varphi<0$ :

$$
\begin{aligned}
& (-1)^{\left\lfloor\frac{\tilde{f}(x)-\varphi-\frac{\pi}{2}}{\pi}\right\rfloor}=(-1)^{\left\lfloor\frac{-x+\tilde{x}^{\mathrm{f}}-\varphi-\frac{\pi}{2}}{\pi}\right\rfloor} \\
= & (-1)^{\left\lfloor\frac{-\left(x-\varphi-\frac{\pi}{2}\right)}{\pi}+1\right\rfloor}=(-1)^{\left\lfloor\frac{x-\varphi-\frac{\pi}{2}}{\pi}\right\rfloor}
\end{aligned}
$$

The equality holds for geometrical reasons.

Induction. to show: $c\left(x_{k}^{c+}-\pi\right)=c\left(\tilde{f}\left(x_{k}^{c-}\right)\right)<c\left(x_{k}^{c-}\right)=-A$.

Induction step. to show: $c(\tilde{f}(x))<c(x) \Longrightarrow c(\tilde{f}(x+\Delta x))<c(x+\Delta x)$ with $\Delta x<0$.

$\Delta x$ is chosen such that segment borders are not crossed. The right side of the equation is considered:

$$
\begin{aligned}
c\left(\tilde{f}\left(x_{i+1}\right)\right)=c\left(-x_{i+1}+\tilde{x}^{f}\right)=c\left(-x_{i}-\Delta x+\tilde{x}^{f}\right)=c\left(\tilde{f}\left(x_{i}\right)+(-\Delta x)\right) & \\
& <c\left(x_{i+1}\right)=c\left(x_{i}+\Delta x\right) \\
\Longleftrightarrow & s\left(\tilde{f}\left(x_{i}\right)\right) A-\sqrt{\left(A-s\left(\tilde{f}\left(x_{i}\right)\right) c\left(\tilde{f}\left(x_{i}\right)\right)\right)^{2}-\varepsilon(-\Delta x)\left(2 \tilde{f}\left(x_{i}\right)+(-\Delta x)\right)} \\
& <s\left(x_{i}\right) A-\sqrt{\left(A-s\left(x_{i}\right) c\left(x_{i}\right)\right)^{2}-\varepsilon(\Delta x)\left(2 x_{i}+\Delta x\right)} \\
\Longleftrightarrow & \left(A-s\left(x_{i}\right) c\left(\tilde{f}\left(x_{i}\right)\right)\right)^{2}-\varepsilon \Delta x\left(-2\left(-x_{i}+\tilde{x}^{f}\right)+\Delta x\right) \\
& >\left(A-s\left(x_{i}\right) c\left(x_{i}\right)\right)^{2}-\varepsilon \Delta x\left(2 x_{i}+\Delta x\right) \\
\Longleftrightarrow & \left(A-s\left(x_{i}\right) c\left(\tilde{f}\left(x_{i}\right)\right)\right)^{2}+2 \varepsilon \Delta x \tilde{x}^{f}>\left(A-s\left(x_{i}\right) c\left(x_{i}\right)\right)^{2}
\end{aligned}
$$

The inequality holds because we assumed $c\left(f\left(x_{i}\right)\right)<c\left(x_{i}\right)<-A$, hence $\left|c\left(f\left(x_{i}\right)\right)\right|>$ $\left|c\left(x_{i}\right)\right|>A$ and $s(x)=s(\tilde{f}(x))$ holds. So far we assumed staying within the same segment, however, because $s(x)=s(\tilde{f}(x))$, incrementally adding $\Delta x$ going from segment border to segment border keeps the above reasoning intact, $c(\tilde{f}(x))<c(x)$ holds true.

Induction start. to show: $c\left(\tilde{f}\left(\tilde{x}^{f}\right)\right)=c(0)<c\left(\tilde{x}^{f}\right)$

This directly follows from section 3.3 .2 (Remarks on monotonicity).

Conclusion. It follows that when passing the left funnel entrance border of funnel $F_{k}^{+}$ the trajectory does not enter the funnel yet: $c\left(x_{k}^{\mathrm{c}+}-\pi\right)=c\left(\tilde{f}\left(x_{k}^{\mathrm{c}-}\right)\right)<c\left(x_{k}^{\mathrm{c}-}\right)=-A$.

Section 3.3.2 (Remarks on monotonicity) stated that $c(x)$ is monotonically increasing for $x>0$ and $c<-A$. It follows that the trajectory will reach $c=-A$ to the 
right of the left funnel entrance border of $F_{k}^{+}: x_{k}^{*-}>x_{k}^{\mathrm{c}+}-\pi$.

\section{(ii.2) To Show: $x_{k}^{\mathrm{c}-}-\pi \leq x_{k}^{*-} ; x_{k}^{*+}$ Lies Left of the Right Entrance of $F_{k}^{\text {in }}$}

Defining $\tilde{\boldsymbol{x}}^{\mathrm{g}}$ and $\tilde{\boldsymbol{g}}(\boldsymbol{x})$. The function $\tilde{g}(x)$ is defined in the following way:

$$
\tilde{g}(x)=-x+\tilde{x}^{\mathrm{g}}
$$

$\tilde{x}^{\mathrm{g}}$ is defined such that it has the same distance to the right border of the funnel entrance $F_{k}^{\mathrm{in}+}$ as the funnel exit point $F_{k}^{\mathrm{o}-}$ from $x=0$.

$$
\tilde{x}^{\mathrm{g}}-\left(x_{k}^{\mathrm{c}+}+\pi\right)=x_{k}^{\mathrm{c}-}-0 .
$$

From the above definition of $\tilde{x}^{g}$ it follows that $\tilde{x}^{g}>0$ :

For $0<\varphi<\pi$ :

$$
\tilde{x}^{g}=2 \varphi>0
$$

For $-\pi<\varphi<0$ :

$$
\tilde{x}^{g}=2 \varphi+2 \pi>0 .
$$

Now we will show that $x$ and $\tilde{g}(x)$ are situated within the same segment type: $s(x)=$ $s(\tilde{g}(x))$. For $0<\varphi<\pi$ as well as $-\pi<\varphi<0$ :

$$
\begin{aligned}
& (-1)^{\left\lfloor\frac{\tilde{g}(x)-\varphi-\frac{\pi}{2}}{\pi}\right\rfloor}=(-1)^{\left\lfloor\frac{-x+\tilde{x}^{g}-\varphi-\frac{\pi}{2}}{\pi}\right\rfloor} \\
& =(-1)^{\left\lfloor\frac{-\left(x-\varphi-\frac{\pi}{2}\right)}{\pi}+1\right\rfloor}=(-1)^{\left\lfloor\frac{x-\varphi-\frac{\pi}{2}}{\pi}\right\rfloor}
\end{aligned}
$$

The equality holds for geometrical reasons.

Induction. to show: When increasing $x, c\left(\tilde{g}\left(x^{\prime}\right)\right)=c\left(x_{k}^{*+}\right)=-A$ occurs before $c\left(x_{k}^{c-}\right)=-A$ and hence $\tilde{g}\left(x_{k}^{c-}\right)=x_{k}^{c+}+\pi>\tilde{g}\left(x^{\prime}\right)=x_{k}^{*+}$.

Induction step. to show: $c(\tilde{g}(x))>c(x) \Longrightarrow c(\tilde{g}(x+\Delta x))>c(x+\Delta x), \Delta x<0$ :

$\Delta x$ is chosen such that segment borders are not crossed. The right hand side is considered:

$$
\begin{aligned}
& c(\tilde{g}(x+\Delta x))<c(x+\Delta x) \\
\Longleftarrow & (A-s(x) c(\tilde{g}(x)))^{2}+2 \varepsilon \Delta x \tilde{x}^{g}>(A-s(x) c(x))^{2} .
\end{aligned}
$$

Assuming $-A \geq c(g(x))>c(x)$ the above inequality follows, given that $\Delta x<0$ and $\tilde{x}^{\mathrm{g}}>0$.

So far it was assumed that $\Delta x$ is chosen such that $x+\Delta x$ remains within the same segment as $x$, however, because $s(x)=s(\tilde{g}(x))$, incrementally adding $\Delta x$ going from segment border to segment border keeps the above reasoning intact, $c(\tilde{g}(x))>c(x)$ holds true.

Induction start. to show: $c\left(\tilde{x}^{g}\right)=c(\tilde{g}(0))>c(0)$ 
This directly follows from 3.3 .2 (Remarks on monotonicity).

Conclusion. While starting at $x=0$ and increasing $|\Delta x|$, the induction above showed that $c(\tilde{g}(x))>c(x)$ has to hold. Hence, before $x_{k}^{\mathrm{c}-}$ with $c\left(x_{k}^{\mathrm{c}-}\right)=-A$ is reached, the point $c(\tilde{g}(x))=-A=c\left(x^{*+}\right)$, when the trajectory reaches $c=-A$ again, has to be passed. Therefore, $x^{*+}<x_{k}^{\mathrm{c}+}+\pi=\tilde{g}\left(x_{k}^{\mathrm{c}-}\right)$. The trajectory reaches $c=-A$ to the left of the right border of $F_{k}^{\text {in }}$.

Short recapitulation. Overall in (ii) we have shown that the trajectory leaving the funnel exit point of the funnel $F_{k}^{-}$reaches between the left and right funnel entrance borders of the funnel $F_{k}^{+}$and hence, have shown that the transition $F_{k}^{\mathrm{o}-} \rightarrow F_{k}^{\text {in+ }}$ occurs. 



\section{Bibliography}

[1] D. M. Abrams and S. H. Strogatz. Chimera states for coupled oscillators. Physical review letters, 93(17):174102, 2004.

[2] R. Adler. A study of locking phenomena in oscillators. Proceedings of the IRE, 34(6):351-357, 1946.

[3] D. J. Amit. Neural networks counting chimes. Proceedings of the National Academy of Sciences, 85(7):2141-2145, 1988.

[4] S. Armstrong, V. Cassone, M. Chesworth, J. R. Redman, and R. V. Short. Synchronization of mammalian circadian rhythms by melatonin. Journal of neural transmission. Supplementum, 21:375-394, 1986.

[5] H. Arnoldt, S. Chang, S. Jahnke, B. Urmersbach, H. Taschenberger, and M. Timme. When less is more: non-monotonic spike sequence processing in neurons. PLoS computational biology, 11(2):e1004002, 2015.

[6] D. Aronov, L. Veit, J. H. Goldberg, and M. S. Fee. Two distinct modes of forebrain circuit dynamics underlie temporal patterning in the vocalizations of young songbirds. Journal of Neuroscience, 31(45):16353-16368, 2011.

[7] P. Ashwin and M. Timme. Unstable attractors: existence and robustness in networks of oscillators with delayed pulse coupling. Nonlinearity, 18(5):2035, 2005.

[8] P. Ashwin, G. Orosz, J. Wordsworth, and S. Townley. Dynamics on networks of cluster states for globally coupled phase oscillators. SIAM Journal on Applied Dynamical Systems, 6(4):728-758, 2007.

[9] A. Baker. Simplicity. In E. N. Zalta, editor, The Stanford Encyclopedia of Philosophy. Metaphysics Research Lab, Stanford University, winter 2016 edition, 2016.

[10] M. Bernardo, C. Budd, A. R. Champneys, and P. Kowalczyk. Piecewise-smooth dynamical systems: theory and applications, volume 163. Springer Science \& Business Media, 2008.

[11] C. Bick, M. Timme, D. Paulikat, D. Rathlev, and P. Ashwin. Chaos in symmetric phase oscillator networks. Physical review letters, 107(24):244101, 2011.

[12] C. Bick, M. J. Panaggio, and E. A. Martens. Chaos in kuramoto oscillator networks. Chaos: An Interdisciplinary Journal of Nonlinear Science, 28(7):071102, 2018. 
[13] D. V. Buonomano and M. M. Merzenich. Temporal information transformed into a spatial code by a neural network with realistic properties. Science, 267(5200): 1028-1030, 1995.

[14] A. J. Catllá, D. G. Schaeffer, T. P. Witelski, E. E. Monson, and A. L. Lin. On spiking models for synaptic activity and impulsive differential equations. SIAM review, 50(3):553-569, 2008.

[15] C. Condat and G. Sibona. Diffusion in a model for active brownian motion. Physica D: Nonlinear Phenomena, 168:235-243, 2002.

[16] V. Daelli and A. Treves. Neural attractor dynamics in object recognition. Experimental brain research, 203(2):241-248, 2010.

[17] M. Denker, M. Timme, M. Diesmann, F. Wolf, and T. Geisel. Breaking synchrony by heterogeneity in complex networks. Physical review letters, 92(7):074103, 2004.

[18] D. Dixon, F. Cummings, and P. Kaus. Continuous "chaotic" dynamics in two dimensions. Physica D: Nonlinear Phenomena, 65(1-2):109-116, 1993.

[19] S. Doi, S. Nabetani, and S. Kumagai. Complex nonlinear dynamics of the hodgkin-huxley equations induced by time scale changes. Biological cybernet$i c s, 85(1): 51-64,2001$.

[20] J. Duncan. An adaptive coding model of neural function in prefrontal cortex. Nature reviews neuroscience, 2(11):820, 2001.

[21] G. B. Ermentrout. Period doublings and possible chaos in neural models. SIAM Journal on Applied Mathematics, 44(1):80-95, 1984.

[22] A. Gal, D. Eytan, A. Wallach, M. Sandler, J. Schiller, and S. Marom. Dynamics of excitability over extended timescales in cultured cortical neurons. Journal of Neuroscience, 30(48):16332-16342, 2010.

[23] D. M. Glasser, J. M. Tsui, C. C. Pack, and D. Tadin. Perceptual and neural consequences of rapid motion adaptation. Proceedings of the National Academy of Sciences, 108(45):E1080-E1088, 2011.

[24] M. Golubitsky and I. Stewart. The symmetry perspective: from equilibrium to chaos in phase space and physical space, volume 200. Springer Science \& Business Media, 2003.

[25] M. Golubitsky, K. Josic, and E. Shea-Brown. Winding numbers and average frequencies in phase oscillator networks. Journal of Nonlinear Science, 16(3): 201-231, 2006.

[26] N. C. Grassly and C. Fraser. Mathematical models of infectious disease transmission. Nature Reviews Microbiology, 6(6):477, 2008.

[27] H. Gruler. Chemokinesis, chemotaxis and galvanotaxis dose-response curves and signal chains. In Biological motion, pages 396-414. Springer, 1990. 
[28] M. R. Guevara, L. Glass, M. C. Mackey, and A. Shrier. Chaos in neurobiology. IEEE Transactions on Systems, Man, and Cybernetics, SMC-13(5):790-798, 1983.

[29] R. Gütig. Spiking neurons can discover predictive features by aggregate-label learning. Science, 351(6277):aab4113, 2016.

[30] D. Hansel, G. Mato, and C. Meunier. Clustering and slow switching in globally coupled phase oscillators. Physical Review E, 48(5):3470, 1993.

[31] D. Hansel, G. Mato, and C. Meunier. Synchrony in excitatory neural networks. Neural computation, 7(2):307-337, 1995.

[32] A. V. Herz and J. J. Hopfield. Earthquake cycles and neural reverberations: collective oscillations in systems with pulse-coupled threshold elements. Physical review letters, 75(6):1222, 1995.

[33] A. L. Hodgkin and A. F. Huxley. A quantitative description of membrane current and its application to conduction and excitation in nerve. The Journal of physiology, 117(4):500-544, 1952.

[34] J. J. Hopfield. Neural networks and physical systems with emergent collective computational abilities. Proceedings of the national academy of sciences, 79(8): 2554-2558, 1982.

[35] E. M. Izhikevich and B. Ermentrout. Phase model. Scholarpedia, 3(10):1487, 2008. doi: 10.4249/scholarpedia.1487. revision \#129938.

[36] S. Jahnke, R.-M. Memmesheimer, and M. Timme. How chaotic is the balanced state? BMC Neuroscience, 10(1):O20, 2009.

[37] T. Kapitaniak. Chaos for Engineers: Theory, Applications, and Control. Springer-Verlag, Berlin Heidelberg, 1998.

[38] H. Kielblock, C. Kirst, and M. Timme. Breakdown of order preservation in symmetric oscillator networks with pulse-coupling. Chaos: An Interdisciplinary Journal of Nonlinear Science, 21(2):025113, 2011.

[39] D. Kleinfeld. Sequential state generation by model neural networks. Proceedings of the National Academy of Sciences of the United States of America, 83(24): 9469, 1986.

[40] R. Kupers and M. Ptito. Compensatory plasticity and cross-modal reorganization following early visual deprivation. Neuroscience $\&$ Biobehavioral Reviews, 41:36$52,2014$.

[41] Y. Kuramoto and D. Battogtokh. Coexistence of coherence and incoherence in nonlocally coupled phase oscillators. arXiv:cond-mat/0210694, 2002.

[42] K. Kürten and J. Clark. Chaos in neural systems. Physics Letters A, 114(7): 413-418, 1986. 
[43] A. C. Kwan. What can population calcium imaging tell us about neural circuits? Journal of neurophysiology, 100(6):2977-2980, 2008.

[44] C. R. Laing. The dynamics of networks of identical theta neurons. The Journal of Mathematical Neuroscience, 8(1):4, 2018.

[45] C. R. Laing. Chaos in small networks of theta neurons. Chaos: An Interdisciplinary Journal of Nonlinear Science, 28(7):073101, 2018.

[46] W. Lauterborn and U. Parlitz. Methods of chaos physics and their application to acoustics. The Journal of the Acoustical Society of America, 84(6):1975-1993, 1988.

[47] I. Lerner and O. Shriki. Internally-and externally-driven network transitions as a basis for automatic and strategic processes in semantic priming: theory and experimental validation. Frontiers in psychology, 5:314, 2014.

[48] M. Linkerhand and C. Gros. Generating functionals for autonomous latching dynamics in attractor relict networks. Scientific reports, 3:2042, 2013.

[49] E. N. Lorenz. Deterministic nonperiodic flow. Journal of the atmospheric sciences, 20(2):130-141, 1963.

[50] M. Lucas, J. Newman, and A. Stefanovska. Stabilization of dynamics of oscillatory systems by nonautonomous perturbation. Physical Review E, 97(4):042209, 2018 .

[51] P. Manz, S. Goedeke, and R.-M. Memmesheimer. Dynamics and computation in mixed spiking networks containing neurons with negative dissipation. arXiv preprint arXiv:1812.09218, 2018.

[52] C. M. Marcus and R. Westervelt. Dynamics of analog neural networks with time delay. In Advances in neural information processing systems, pages 568-576, 1989.

[53] H. Markram, K. Meier, T. Lippert, S. Grillner, R. Frackowiak, S. Dehaene, A. Knoll, H. Sompolinsky, K. Verstreken, J. DeFelipe, et al. Introducing the human brain project. Procedia Computer Science, 7:39-42, 2011.

[54] R. M. May. Simple mathematical models with very complicated dynamics. $N a$ ture, 261(5560):459, 1976.

[55] L. Mazzucato, A. Fontanini, and G. La Camera. Dynamics of multistable states during ongoing and evoked cortical activity. Journal of Neuroscience, 35(21): 8214-8231, 2015.

[56] F. Menghini, N. van Rijsbergen, and A. Treves. Modelling adaptation aftereffects in associative memory. Neurocomputing, 70(10-12):2000-2004, 2007.

[57] R. E. Mirollo and S. H. Strogatz. Synchronization of pulse-coupled biological oscillators. SIAM Journal on Applied Mathematics, 50(6):1645-1662, 1990. 
[58] G. Mongillo, O. Barak, and M. Tsodyks. Synaptic theory of working memory. Science, 319(5869):1543-1546, 2008.

[59] M. Monteforte and F. Wolf. Dynamical entropy production in spiking neuron networks in the balanced state. Physical review letters, 105(26):268104, 2010.

[60] F. Moon and P. J. Holmes. A magnetoelastic strange attractor. Journal of Sound and Vibration, 65(2):275-296, 1979.

[61] H. Nakano and T. Saito. Basic dynamics from a pulse-coupled network of autonomous integrate-and-fire chaotic circuits. IEEE Transactions on Neural Networks, 13(1):92-100, 2002.

[62] F. S. Neves and M. Timme. Controlled perturbation-induced switching in pulsecoupled oscillator networks. Journal of Physics A: Mathematical and Theoretical, 42(34):345103, 2009.

[63] F. S. Neves and M. Timme. Computation by switching in complex networks of states. Physical review letters, 109(1):018701, 2012.

[64] F. S. Neves, M. Voit, and M. Timme. Noise-constrained switching times for heteroclinic computing. Chaos: An Interdisciplinary Journal of Nonlinear Science, 27(3):033107, 2017.

[65] V. Nosov, H. Dominguez, J. Ortega-Herrera, and J. Meda-Campana. Complex dynamics and chaos in commutable pendulum. Revista mexicana de física, 58 (1):6-12, 2012.

[66] T. I. Panagiotaropoulos, V. Kapoor, N. K. Logothetis, and G. Deco. A common neurodynamical mechanism could mediate externally induced and intrinsically generated transitions in visual awareness. PloS one, 8(1):e53833, 2013.

[67] U. Parlitz, A. Pikovsky, M. Rosenblum, and J. Kurths. Schwingungen im Gleichtakt. Physik Journal, 5(5):33-40, 2006.

[68] D. Pazó and E. Montbrió. Low-dimensional dynamics of populations of pulsecoupled oscillators. Physical Review X, 4(1):011009, 2014.

[69] S. M. Plaza, L. K. Scheffer, and D. B. Chklovskii. Toward large-scale connectome reconstructions. Current opinion in neurobiology, 25:201-210, 2014.

[70] S. Renals and R. Rohwer. A study of network dynamics. Journal of Statistical Physics, 58(5-6):825-848, 1990.

[71] P. Romanczuk, M. Bär, W. Ebeling, B. Lindner, and L. Schimansky-Geier. Active brownian particles. The European Physical Journal Special Topics, 202(1):1-162, 2012.

[72] O. E. Rössler. An equation for continuous chaos. Physics Letters A, 57(5): 397-398, 1976.

[73] M. Schienbein and H. Gruler. Langevin equation, fokker-planck equation and cell migration. Bulletin of Mathematical Biology, 55(3):585-608, 1993. 
[74] F. Schweitzer, W. Ebeling, and B. Tilch. Complex motion of brownian particles with energy depots. Physical Review Letters, 80(23):5044, 1998.

[75] S. Shoham, D. H. O'Connor, and R. Segev. How silent is the brain: is there a "dark matter" problem in neuroscience? Journal of Comparative Physiology A, 192(8):777-784, 2006.

[76] H. Sompolinsky, A. Crisanti, and H.-J. Sommers. Chaos in random neural networks. Physical review letters, 61(3):259-262, 1988.

[77] S. Song, H. Yao, and A. Treves. A modular latching chain. Cognitive neurodynamics, 8(1):37-46, 2014.

[78] M. E. Spira and A. Hai. Multi-electrode array technologies for neuroscience and cardiology. Nature nanotechnology, 8(2):83, 2013.

[79] J. Sprott. A simple chaotic delay differential equation. Physics Letters A, 366 $(4-5): 397-402,2007$.

[80] J. C. Sprott. Elegant chaos: algebraically simple chaotic flows. World Scientific, 2010 .

[81] I. Stewart, M. Golubitsky, and M. Pivato. Symmetry groupoids and patterns of synchrony in coupled cell networks. SIAM Journal on Applied Dynamical Systems, 2(4):609-646, 2003.

[82] S. H. Strogatz. Nonlinear Dynamics and Chaos: With Applications to Physics, Biology, Chemistry, and Engineering. Westview Press, Boulder, CO, 2 edition, 2015.

[83] M. Timme and F. Wolf. The simplest problem in the collective dynamics of neural networks: is synchrony stable? arXiv preprint arXiv:0810.4472, 2008.

[84] M. Timme, F. Wolf, and T. Geisel. Coexistence of regular and irregular dynamics in complex networks of pulse-coupled oscillators. Physical review letters, 89(25): 258701, 2002 .

[85] M. Timme, F. Wolf, and T. Geisel. Unstable attractors induce perpetual synchronization and desynchronization. Chaos: An Interdisciplinary Journal of Nonlinear Science, 13(1):377-387, 2003.

[86] M. Timme, T. Geisel, and F. Wolf. Speed of synchronization in complex networks of neural oscillators: analytic results based on random matrix theory. Chaos: An Interdisciplinary Journal of Nonlinear Science, 16(1):015108, 2006.

[87] A. Treves. Frontal latching networks: a possible neural basis for infinite recursion. Cognitive neuropsychology, 22(3-4):276-291, 2005.

[88] C. Van Vreeswijk and H. Sompolinsky. Chaos in neuronal networks with balanced excitatory and inhibitory activity. Science, 274(5293):1724-1726, 1996.

[89] C. v. Vreeswijk and H. Sompolinsky. Chaotic balanced state in a model of cortical circuits. Neural computation, 10(6):1321-1371, 1998. 
[90] X. Wang. Period-doublings to chaos in a simple neural network: An analytical proof. Complex Systems, 5(4):425-444, 1991.

[91] Wikipedia contributors. Evolution - Wikipedia, the free encyclopedia. https:// en.wikipedia.org/w/index.php?title=Evolution\&oldid=897605852, 2019. [Online; accessed 24-May-2019].

[92] Wikipedia contributors. Langton's ant - Wikipedia, the free encyclopedia. https://en.wikipedia.org/w/index.php?title=Langton\%27s_ant\& oldid=896171281, 2019. [Online; accessed 24-May-2019].

[93] Wikipedia contributors. Peppered moth - Wikipedia, the free encyclopedia. https://en.wikipedia.org/w/index.php?title=Peppered_moth\&oldid= 897531479, 2019. [Online; accessed 20-May-2019].

[94] Wikipedia contributors. Synapse - wikipedia, die freie enzyklopädie. https: //de.wikipedia.org/w/index.php?title=Synapse\&oldid=185643463, 2019. [Online; accessed 24-May-2019].

[95] Y.-Y. Xu. Interference of quantum chaotic systems in phase space. Communications in Theoretical Physics, 60(4):453, 2013.

[96] A. A. Zaher and A. Abu-Rezq. On the design of chaos-based secure communication systems. Communications in Nonlinear Science and Numerical Simulation, 16(9):3721-3737, 2011. 



\section{Acknowledgements}

At this point it is time to thank everyone, who has supported me during my $\mathrm{PhD}$, whether through emotional support or action or just by walking some steps with me during this phase of life. Even though it is impossible to mention everyone by name I would like to highlight a few humans.

My supervisor Marc, thank you for taking up the adventure of supervising a psychologist. Thank you for a lot of support and patience. I greatly appreciate you as a supervisor who pushes the science forward while not forgetting the human side of things.

Thank you, Nic, you were there in difficult times and helped me grow. We had a great time together. This means a lot to me. Suresh, I thank you for your support during the time our paths were still joined.

Some friendships were newly formed, others deepened. Carolin, thermodynamics forms strong bonds, I am glad we met and I am looking forward to great times with you. Felix, I really enjoyed gloomy and cosy evenings with people and guitar music, working sessions and walks around the Faßberg forest with Jana, thank you both for broadening my perspective and being there. Jule, traveling with you is awesome and easy and I hope we will go for a few more adventures. Christine, thank you for all your support and advice in life matters. Malte and Benjamin, thanks for answering physics questions and many board game afternoons. I thank all the network dynamics people and Bunsenstraße people for yellow kitchen cooking events and being a friendly and fun community. Xiaozhu, thank you for hosting me and giving me insights into chinese culture. Nora, thank you for making work life more creative. Also I would like to mention the neuroscience people I met at the institute or at conferences, especially Sven and Dimitra. I would especially like to thank my flatmates Marco, Flo, Tomos and Ana. It is so valuable to have a place where you feel at home and you make it so. And then there are the Hüttenfahrer and Quartettsänger, Refugee Law Clinic organizers, Pink Fluid and the institute 'metal' band, the Blaue Sänger Orchestra.

Thanks to my thesis committee, Prof. Reiner Kree and Prof. Florentin Wörgötter for feedback, steering and ideas. Thanks to the members of the examination board.

Thanks also to the staff of the MPI DS and to the PBCS office, it was not easy finding the right place between physics and psychology. I would like to acknowledge the financial support through the IMPRS PBCS excellence stipend.

And finally, thanks to my family, who sparked and supported my curiosity in science. 